\title{
Relation Between Urbanization and Water Quality of Streams in the Austin Area, Texas
}

By Jack $E$. Veenhuis and Raymond M. Slade, Jr.

U.S. GEOLOGICAL SURVEY

Water-Resources Investigations Report 90-4107

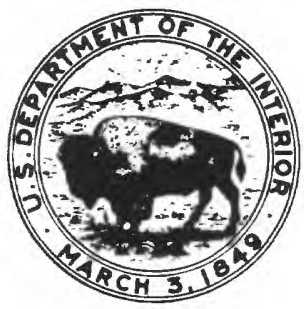

Prepared in cooperation with the CITY OF AUSTIN 


\section{U.S. DEPARTMENT OF THE INTERIOR \\ MANUEL LUJAN, JR., Secretary \\ U.S. GEOLOGICAL SURVEY \\ Dallas L. Peck, Director}

For additional information write to:

District Chief

U.S. Geological Survey

8011 Cameron Rd., Bldg. 1

Aust in, TX 78753
Copies of this report can be purchased from:

U.S. Geological Survey Books and Open-File Reports Section Box 25425, Federal Center, Bldg. 810 Denver, CO 80225 
Abstract--

Introduction-_-

Purpose and scope-_-_-_ 2

Previous investigations-_-_-_- 2

Location and description of the study area-_-_-_-_- 5

Acknowledgments-_-_-_-_-_- 6

Description of drainage basins and sample sites-_-_-_-_-_-_-_-_-_-_-_ 6

Approach--_-_-_-_-_-_-_ 8

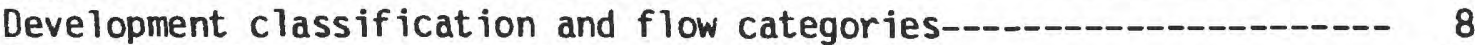

Data collection and analysis-_-_-_ 10

Statistical comparisons-_- 12

Distribution of sample discharge-_-_-_-_- 13

Distribution of water-quality properties and constituents--_-_-_-_-_--_- 17

Physical organics and inorganics-_- 17

Dissolved solids-_-_-__- 17

Suspended solids--_-_-_. 19

Biochemical oxygen demand-_-_-_-_-_-_-_-_ 22

Total organic carbon-_-_-_-_ 22

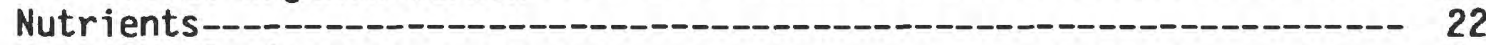

Total nitrogen--_-_-_-_-_-_-_- 25

Total phosphorus-_-_-_- 25

Indicator bacteria--_-_- 28

Fecal coliforms-_-_-_- 28

Fecal streptococci-_- 30

Ratios of fecal coliforms to fecal streptococci-_-_-_-_-_-_- 30

Inorganic trace elements-_- 30

Synthetic organic compounds-_-_- 40

Industrial compounds--_- 45

Insecticides-_- 45

Herbicides-_-_-_- 45

Radiochemical constituents--_-_- 47

Relation between urbanization and water quality-_-_-_-_-_-_-_-_-_ 47

Comparisons among five sites with automatic samplers---_-_-_-_-_-- 47

Summary-

Comparisons among four development classifications--_-_-_-_-_-_-_-_-- 49

\section{ILLUSTRATIONS}

[Plate is in pocket]

Plate 1. Map showing location of water-quality sample sites with streets and roads.

Figure 1. Map showing location of study area-_- 3

2. Map showing location of water-quality sample sites--.---_----- 4

3. Graph showing relation between drainage area and percent of impervious cover in the basin upstream from sample sites---_- 
Figures 4-12. Boxplots showing the distribution, by flow category and sample site, of:

4. Discharge at time of sample collection-........... 14

5. Dissolved-solids concentrations-_................. 18

6. Suspended-solids concentrations-_._._._._._._._. 20

7. Biochemical oxygen demand concentrations-_.........- 23

8. Total organic carbon concentrations_............... 24

9. Tota1 nitrogen concentrations-_._. 26

10. Total phosphorus concentrations__._. 27

11. Feca1-coliform densities-_._. 29

12. Feca1-streptococcal densities-_.................. 31

13-21. Boxplots showing the distribution, by sample site, of:

13. Dissolved arsenic concentrations-_.............. 33

14. Dissolved barium concentrations_................... 34

15. Dissolved copper concentrations_................. 37

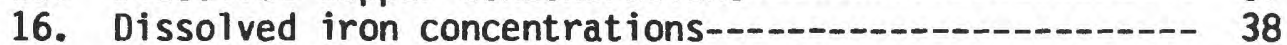

17. Dissolved manganese concentrations_.............. 39

18. Dissolved zinc concentrations-_._. 41

19. Dissolved boron concentrations__._. 42

20. Dissolved stront ium concentrations_............... 43

21. Total diazinon concentrations-_._._. 46

22-29. Boxplots showing the distribution, by flow category and development classification, of:

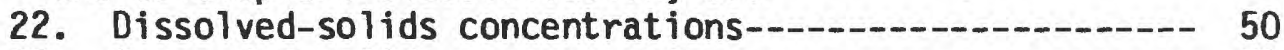

23. Suspended-solids concentrations_._._._._._._._._. 51

24. Biochemical oxygen demand concentrations-_._._._.-. 54

25. Total organic carbon concentrations-_............. 55

26. Total nitrogen concentrations-_._. 56

27. Total phosphorus concentrations-_._. 57

28. Fecal-coliform densities-_._. 58

29. Fecal-streptococcal densities-_._. 60

\section{TABLES}

Table 1. Selected drainage-basin characteristics, period of record, and number of samples for sample sites-

2. Permitted discharge and permitted quality of effluent for wastewater facilities within drainage basins of sample sites

3. Median discharges and concentrations and densities of selected water-quality properties and constituents from rising stage and falling stage of stormflow and from base flow at sample sites-

4. Relation between dissolved-solids concentration and specific conductance at selected sample sites-_-_-_-_-_-_- 21

5. Summary of inorganic trace elements_...... 35

6. Summary of synthetic organic compounds_............ 44

7. Summary of radiochemical data by sample site-_._._._._._._. 48

8. Median concentrations and densities of selected water-quality properties and constituents for four development classifications 
Factors for converting inch-pound units to metric (International System) units are given in the following table:

\begin{tabular}{lcl}
\hline Multiply inch-pound unit & By & To obtain metric unit \\
\hline cubic foot per second $\left(\mathrm{ft}^{3} / \mathrm{s}\right)$ & 0.02832 & cubic meter per second \\
degree Fahrenheit $\left({ }^{\circ} \mathrm{F}\right)$ & $5 / 9\left({ }^{\circ} \mathrm{F}-32\right)$ & degree Celsius $\left({ }^{\circ} \mathrm{C}\right)$ \\
foot (ft) & 0.3048 & meter \\
inch (in.) & 25.40 & millimeter \\
mile (mi) & 1.609 & kilometer \\
pound per day (lb/d) & 0.4536 & kilogram per day \\
square mile $\left(\mathrm{mi}^{2}\right)$ & 2.590 & square kilometer \\
\hline
\end{tabular}

Sea level: In this report "sea level" refers to the National Geodetic Vertical Datum of 1929 (NGVD of 1929)--a geodetic datum derived from a general adjustment of the first-order level nets of both the United States and Canada, formerly called Sea Level Datum of 1929. 


\title{
RELATION BETWEEN URBANIZATION AND WATER QUALITY OF
}

\author{
STREAMS IN THE AUSTIN AREA, TEXAS
}

By

\author{
Jack E. Veenhuis and Raymond M. Slade, Jr.
}

\section{ABSTRACT}

Selected water-quality properties and constituents of stormflow and base flow were compared to determine the relation between the degree of urbanization and the water quality in a drainage basin. Samples were collected during three flow categories (rising stage and falling stage of stormflow and base flow) at 18 sites on 11 streams. The degree of urbanization is indicated by grouping the sample sites by development based on the percent of impervious cover in the drainage basin. Four development classifications--rural, mostly rural, partly urban, and urban--were identified based on ranges of impervious cover in the drainage basins. For each site and development classification, the concentrations and densities in samples collected during the rising stage and falling stage of stormflow and during base flow were compared. Samples were collected for physical organics and inorganics, nutrients, indicator bacteria, inorganic trace elements, synthetic organic compounds, and radiochemical constituents.

Except for dissolved solids, the concentrations in samples collected during the rising stage of stormflow were larger than in samples collected during the falling stage. The concentrations in stormflow were larger than in base flow. The concentrations and densities of suspended solids, biochemical oxygen demand, total organic carbon, total nitrogen, total phosphorus, fecal coliforms, and fecal streptococci increased with increased impervious cover.

For the five sample sites that had a sufficient number of samples from each flow category for statistical comparisons, the median concentrations in samples collected during the rising stage and falling stage of stormf low were significantly larger than the base-flow median concentrations. In addition, the concentrations in samples collected during the rising stage were more variable and significantly larger than in those collected during the falling stage of stormflow. Statistical differences between the median concentrations in samples collected during the rising stage and falling stage of stormflow were not evident for the site with the mostly rural drainage basin.

Except for dissolved solids, the median concentrations in samples collected during the rising stage and falling stage of stormflow increased with increasing development classifications. For base flow, the median concentrations were also larger for more urban classifications than for the other classifications.

The ratio of the number of samples with detectable concentrations to the total number of samples analyzed for 18 inorganic trace elements and the concentrations of many of these minor constituents increased with increasing development classifications. Twenty-two of the 42 synthet ic organic compounds for which samples were analyzed were detected in one or more samples. The compounds were detected more frequently and in larger concentrations at the sites with more urban classifications. 


\section{INTRODUCTION}

The Austin metropolitan area is one of the fastest growing areas in the nation. In 1982, approximately 577,000 people lived in the Austin metropolitan area, and a population of 1.9 million by the year 2020 was projected by the City of Austin, Planning Department (1982). Much of the runoff from precipitation in the Austin area flows into reservoirs that are sources of municipal and recreation water for Aust in and several small cities near Austin.

In 1975, the U.S. Geological Survey, in cooperation with the city of Austin, began a hydrologic investigation of drainage basins in the Austin area (fig. 1) to determine whether urban runoff has caused changes in water quality. The study included the gaging of precipitation and streamflow and the collection and analysis of water-quality samples at the streamflow-gaging sites. These samples were collected to provide data to determine the waterquality characteristics of stormflow and base flow for drainage basins with different degrees of urbanization. In this study, the degree of urbanization was indicated by grouping sample sites into development classifications according to the estimated percent of impervious cover of each basin. In October 1986, the number of sample sites and the frequency of sample collection were decreased.

\section{Purpose and Scope}

This report presents and compares the results of water-quality sampling of stormflow and base flow from drainage basins ranging from rural to urban. The data include samples collected by the Geological Survey from 18 sites on 11 streams in the Austin area (fig. 2) from 1974 to 1985.

The water-quality properties and constituents chosen for analysis included those that are important for determining the suitability of water for most purposes and that are believed to be influenced by land use. These properties and constituents are: (1) Physical organics and inorganics, (2) nutrients, (3) indicator bacteria, (4) inorganic trace elements, (5) synthetic organic compounds, and (6) radiochemical constituents.

\section{Previous Investigations}

The data used in this report were published by the Geological Survey in annual reports entitled "Hydrologic data for urban studies in the Austin, Texas, metropolitan area." These reports present all the precipitation, streamflow, and water-quality data collected from 1974 to 1986.

Numerous investigations of streamflow quality have been conducted by city, county, State, Federal, educational, and private agencies in the Austin area. In addition to the Geological Survey program, at least three programs of streamflow sampling currently (1990) exist in Austin. The Austin-Travis County Health Department has conducted a sampling program at many sites since 1968. In 1982, the Lower Colorado River Authority began sampling at seven sites on four streams in Austin. The Department of Environmental Protection of the city of Aust in began sampling runoff at 13 sites in 1984. Many of the city's sampling sites monitor inflow or outflow to runoff-filtering systems on small creeks. 


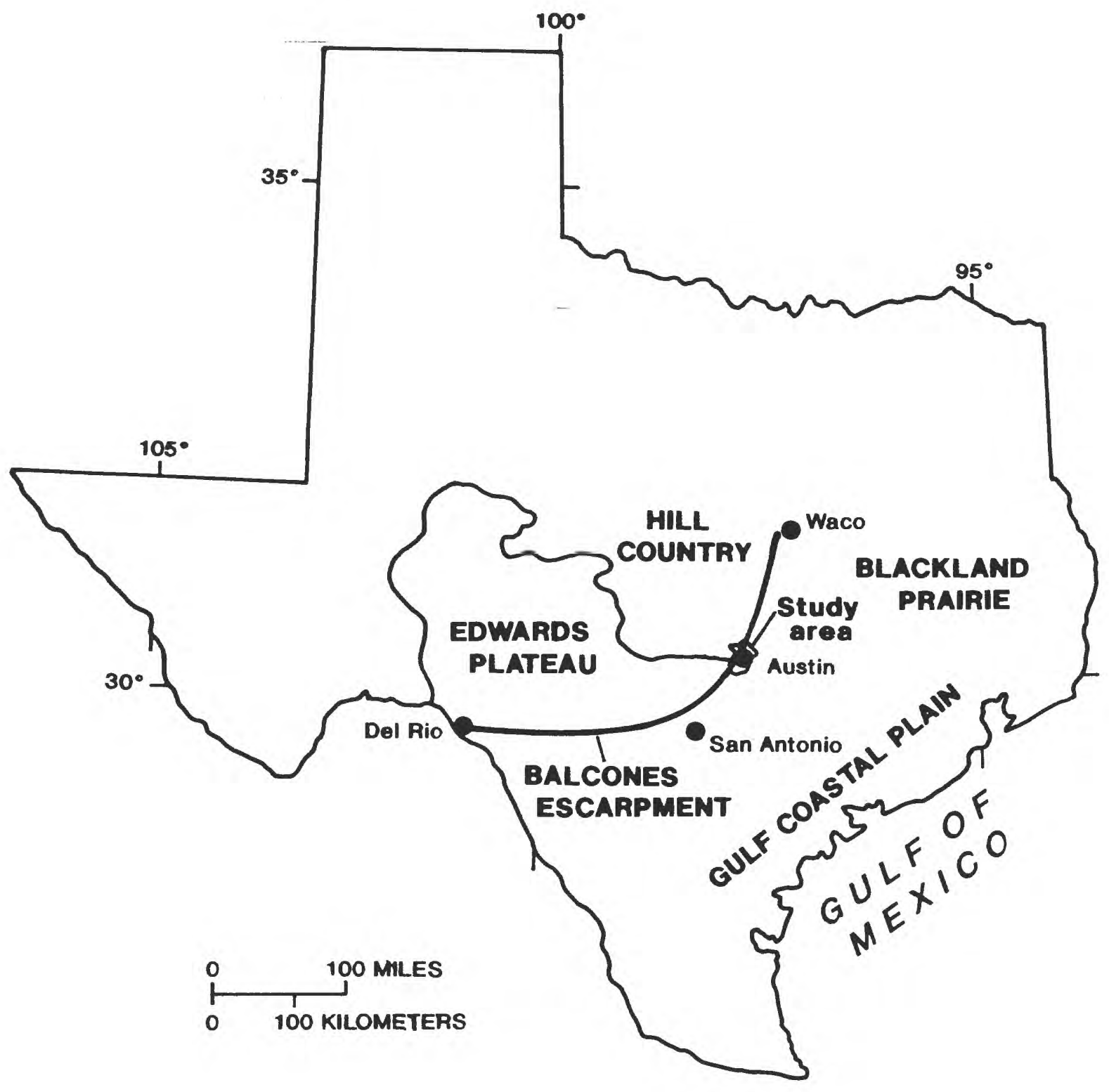

Figure 1.--Location of study area. 


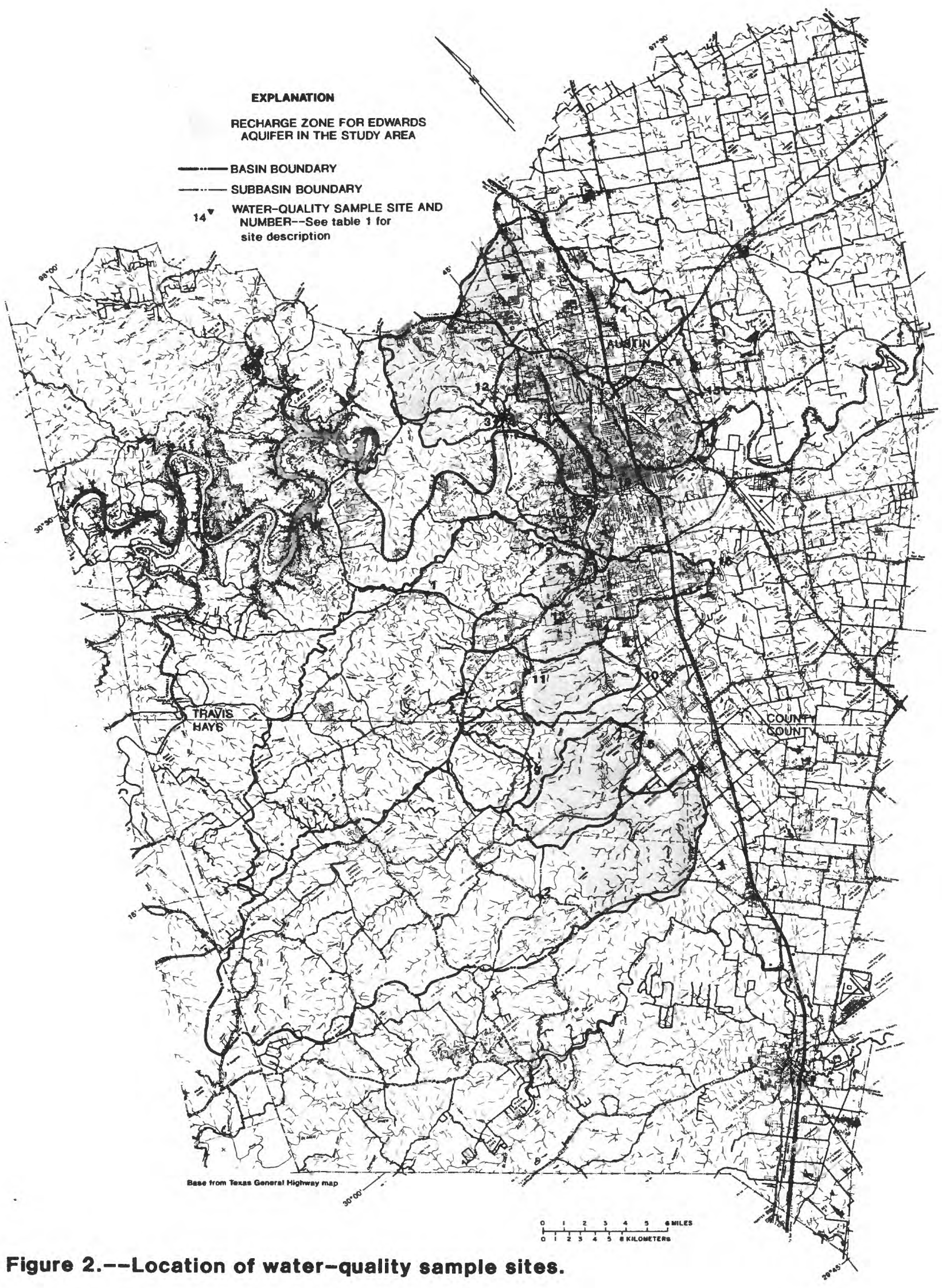


Several reports-concerning streamflow quality in Aust in have been prepared. The results of water-quality investigations for three small creeks, Turkey Creek (a tributary to Lake Austin) and two unnamed creeks, were prepared for the Nationwide Urban Runoff Program (NURP) by EngineeringScience, Inc. (1982). The city of Austin, using precipitation-runoff and water-quality data from the Geological Survey, presented estimations of the effects of urban development on stormwater runoff and runoff-pollutant loads (City of Austin, Watershed Management Division, Public Works Department, 1984). Andrews and others (1984) presented the effects of storm runoff on the quality of Barton Springs and the associated Edwards aquifer. The effects of runoff-filtering systems on the quality of runoff from two sites in Aust in were presented by Welborn and Veenhuis (1987). Since 1982, one-time waterquality surveys for many streams in the Aust in area have been conducted by the Texas Water Commission. The results of these surveys were published, by drainage basin, in many individual reports.

Reports presenting the effects of urbanization on the water quality of runoff have been prepared for many regions of the United States during the past 15 years. The results of the NURP were presented by the U.S. Environmental Protection Agency (1983). Several reports of area-specific investigations of the quality of urban runoff, many of which are part of NURP, have been prepared by the Geological Survey and other agencies (Blevins, 1984; Ellis and Alley, 1979; Ellis and others, 1983; Fisher and Katz, 1984; Harms and others, 1983; and Miller and McKenzie, 1978).

\section{Location and Description of the Study Area}

The study area includes 11 drainage basins in the Aust in metropolitan area. Austin is located in Travis County (fig. 2), which is approximately 150 mi northwest of the Gulf of Mexico in south-central Texas (fig. 1). The area extends from the Hill Country at the eastern edge of the Edwards Plateau across the Balcones Escarpment to the Blackland Prairie region of Texas. The altitude of the study area ranges from about $400 \mathrm{ft}$ above sea level at the downstream end of Boggy Creek to about $1,600 \mathrm{ft}$ above sea leve 1 at the headwaters of Onion Creek. Soils are usually thin over hard limestone in the western part of the study area. Soft limestone and shale are found in the vicinity of the Balcones fault zone. Soils 12 in. or more in thickness overlying shale are found in the eastern part of the study area. The soils are predominately clay or silty clay of slight permeability, except along the flood plain and alluvial terraces of the Colorado River where soils are thicker, more sandy, and more permeable. The geology of the Aust in area is described by Garner and Young (1976). Detailed information about soils in Travis County is presented by the U.S. Department of Agriculture (1974).

The climate of Aust in is characterized by short mild winters, long moderately hot summers, moderately high humidity, and southerly winds. The mean-annual temperature, based on 1941-70, is $71^{\circ} \mathrm{F}\left(21.5^{\circ} \mathrm{C}\right)$; the meanmaximum temperature for July is $95^{\circ} \mathrm{F}\left(35^{\circ} \mathrm{C}\right)$; and the mean-minimum temperature for January is $41^{\circ} \mathrm{F}\left(5.0^{\circ} \mathrm{C}\right)$ (Carr, 1967). The average growing season is 270 days.

Mean-annual precipitation at the National Weather Service (NWS) gage in Austin was about 32 in., and annual precipitation ranged from 11 to 51 in. during 1928-82. Precipitation is distributed somewhat eveniy throughout the year with slightly larger amounts in the spring and early fall months. 
Individual storm precipitation, as well as annual total precipitation, can vary considerably within the study area. Mean-annual pan evaporation from the NWS gage in Aust in is 73.82 in. for 1916-79 (Farnsworth, 1982).

\section{Acknowledgments}

The authors wish to thank the personnel of the Watershed Management Division of the city of Austin Public Works Department (currently staff of the Department of Environmental and Conservational Services) for their valuable assistance during the study. The authors also acknowledge Michael E. Dorsey and Emma M. McPherson of the Geological Survey, Texas District, who collected and analyzed the water-quality samples, frequently outside of normal working hours.

\section{DESCRIPTION OF DRAINAGE BASINS AND SAMPLE SITES}

Water samples were collected at 18 sites on 11 streams in the Austin area from 1974 to 1985 (fig. 2). Streams and locations of sample sites are shown with the streets and roads in plate 1 to give an indication of the extent and degree of urbanization upstream from each sample site. The name and a description of each of the sample sites are given in table 1. The table also includes the number of samples collected at each site.

Most streamflow is the result of runoff from rainstorms at each of the 11 streams sampled. During prolonged periods of little or no precipitation, many streams have minimal flow. Because of their larger drainage areas, the larger streams, Walnut Creek, Barton Creek, and Onion Creek, have longer periods of sustained flow after storms than do the other sampled streams. Small springs located in the Bu11 Creek drainage basin issue from the Edwards aquifer and sustain streamflow long after stormflow ceases. Likewise, springs from the Trinity aquifer constitute much of the base flow at the upstream sample sites on Barton Creek, Bear Creek, and Slaughter Creek. Small springs also provide a part of the base flow of Shoal Creek. The base flows for the streams with the more urban drainage areas are supplemented by water from excess seasonal lawn and garden irrigation, the draining of municipal swimming pools, and other miscellaneous sources discharged through storm sewers.

Barton Creek, Williamson Creek, Slaughter Creek, Bear Creek, Little Bear Creek, and Onion Creek flow over part of the Edwards aquifer recharge zone (fig. 2) associated with Barton Springs, which is located near the mouth of Barton Creek (Baker and others, 1986). The Barton Springs part of the recharge zone is south of the Colorado River. Much of the base flow as well as part of the stormflow of these streams is lost to the Edwards aquifer as the streams pass over the recharge zone. Two sites on Barton Creek (sites 1 and 5) are upstream from the recharge zone; the site on Barton Creek at Loop 360 (site 7) is located within the recharge zone. The upstream sites on Onion Creek (site 2), Slaughter Creek (site 11), and Williamson Creek (site 13) are upstream from the recharge zone; the lower sites (sites 4, 10, and 16, respectively) are downstream from it. The site on Bear Creek (site 9) is upstream from the recharge zone; the site on Little Bear Creek (site 6) is downstream from it.

Several of the sites are downstream from wastewater-treatment facilities. Permitted discharge and permitted quality of effluent are given for each 
Table 1.--Selected drainage-basin characteristics, period of record, and number of samples for sample sites

[mi ${ }^{2}$, square miles; <, less than]

\begin{tabular}{|c|c|c|c|c|c|c|c|c|c|}
\hline $\begin{array}{l}\text { Sample } \\
\text { site } \\
\text { number } \\
\text { (fig. 2) } \\
\end{array}$ & $\begin{array}{l}\text { Station } \\
\text { number }\end{array}$ & Station name & $\begin{array}{c}\text { Impervious } \\
\text { cover } \\
\text { (percent) }\end{array}$ & $\begin{array}{l}\text { Develop- } \\
\text { ment } \\
\text { classi- } \\
\text { fication } \\
\end{array}$ & $\begin{array}{c}\text { Orain- } \\
\text { age } \\
\text { area } \\
\left(m i^{2}\right)\end{array}$ & $\begin{array}{l}\text { Period } \\
\text { of } \\
\text { record } \\
\text { analyzed } \\
\end{array}$ & $\begin{array}{l}\frac{\mathrm{Nu}}{\text { stor }} \\
\text { Rising } \\
\text { stage }\end{array}$ & $\begin{array}{l}\text { mber of s } \\
\text { mflow } \\
\text { FaTling } \\
\text { stage }\end{array}$ & $\frac{\text { samples }}{\text { Base ftow }}$ \\
\hline 1 & 08155200 & $\begin{array}{l}\text { Barton Creek at U.S. Highway } 71 \\
\text { near Oak Hill, Tex. }\end{array}$ & $<1$ & Rural & 89.7 & $1978-82$ & 1 & 12 & 10 \\
\hline 2 & 08158700 & Onion Creek near Driftwood, Tex. & $<1$ & do. & 124 & $1974-85$ & 2 & 14 & 59 \\
\hline 3 & 08154750 & $\begin{array}{l}\text { West Bull Creek at Loop } 360 \\
\text { near Aust in, Tex. }\end{array}$ & 2 & $\begin{array}{l}\text { Most ly } \\
\text { rural }\end{array}$ & 6.77 & $1978-79$ & 0 & 1 & 9 \\
\hline 4 & 08158800 & Onion Creek near Buda, Tex. & 2 & do. & 166 & $1978-83$ & 5 & 11 & 9 \\
\hline $1_{5}$ & 08155260 & $\begin{array}{l}\text { Barton Creek near Camp Craft } \\
\text { Road, Austin, Tex. }\end{array}$ & 3 & do. & 109 & $1983-85$ & 22 & 4 & 4 \\
\hline 6 & 08158825 & $\begin{array}{l}\text { Little Bear Creek at Farm Road } \\
1626 \text { near Manchaca, Tex. }\end{array}$ & 4 & do. & 21.0 & $1978-83$ & 2 & 7 & 1 \\
\hline $1_{7}$ & 08155300 & $\begin{array}{l}\text { Barton Creek at Loop } 360 \text {, } \\
\text { Aust in, Tex. }\end{array}$ & 4 & do. & 116 & $1979-85$ & 34 & 19 & 8 \\
\hline 8 & 08154760 & $\begin{array}{l}\text { Bul1 Creek at Farm Road } 2222 \\
\text { near Austin, Tex. }\end{array}$ & 6 & do. & 30.4 & $1975-78$ & 3 & 9 & 16 \\
\hline 9 & 0.8158810 & $\begin{array}{l}\text { Bear Creek below Farm Road } 1826 \\
\text { near Driftwood, Tex. }\end{array}$ & 7 & do. & 12.2 & $1978-85$ & 1 & 10 & 16 \\
\hline 10 & 08158860 & $\begin{array}{l}\text { Slaughter Creek at Farm Road } \\
2304 \text { near Austin, Tex. }\end{array}$ & 9 & $\begin{array}{l}\text { Partly } \\
\text { urban }\end{array}$ & 23.1 & $1979-83$ & 1 & 5 & 3 \\
\hline 11 & 08158840 & $\begin{array}{l}\text { Slaughter Creek at Farm Road } \\
1826 \text { near Aust in, Tex. }\end{array}$ & 10 & do. & 8.24 & $1983-85$ & 1 & 3 & 5 \\
\hline${ }^{1} 12$ & 08154700 & $\begin{array}{l}\text { Bull Creek at Loop } 360 \\
\text { near Austin, Tex. }\end{array}$ & 10 & do. & 22.3 & $1978-85$ & 28 & 26 & 17 \\
\hline${ }_{13}$ & 08158920 & $\begin{array}{l}\text { Williamson Creek at Oak Hill, } \\
\text { Tex. }\end{array}$ & 12 & do. & 6.30 & $1974-85$ & 26 & 37 & 36 \\
\hline 14 & 08158200 & $\begin{array}{l}\text { Walnut Creek at Dessau Road, } \\
\text { Aust in, Tex. }\end{array}$ & 15 & do. & 26.2 & $1979-85$ & 1 & 11 & 7 \\
\hline 15 & 08158600 & $\begin{array}{l}\text { Walnut Creek at Webberville } \\
\text { Road, Austin, Tex. }\end{array}$ & 17 & do. & 51.3 & $1976-85$ & 6 & 19 & 26 \\
\hline 16 & 08158970 & $\begin{array}{l}\text { Williamson Creek at Jimmy Clay } \\
\text { Road, Aust in, Tex. }\end{array}$ & 20 & do. & 27.6 & $1975-85$ & 13 & 16 & 30 \\
\hline $1_{17}$ & 08158050 & $\begin{array}{l}\text { Boggy Creek at U.S. Highway } 183 \text {, } \\
\text { Aust in, Tex. }\end{array}$ & 40 & Urban & 13.1 & $1975-85$ & 62 & 53 & 32 \\
\hline $1_{18}$ & 08156800 & $\begin{array}{l}\text { Shoal Creek at } 12 \text { th Street, } \\
\text { Aust in, Tex. }\end{array}$ & 42 & do. & 12.3 & $1975-85$ & 55 & 60 & 16 \\
\hline
\end{tabular}

1 Equipped with automatic sampler. 
wastewater facility in table 2 (Texas Water Commission, written commun., 1987). Outflow from the two wastewater-treatment facilities on Williamson Creek probably constitutes most of the base flow at the site at Oak Hill (site 13), whereas the two facilities on Walnut Creek contribute only a small part of the normal base flow. Because the reach between the facility on Slaughter Creek and the downstream site (10) overlies the recharge zone, little, if any, effluent reaches the site. Most wastewater-treatment facilities over or near the recharge zone are issued no-discharge permits requiring them to dispose of effluents by irrigation. Only the Williamson Creek treatment plant, operated by the city of Austin, disposed of treated effluent using irrigation and discharge. The treatment plant is within the Williamson Creek drainage bas in immediately upstream of site 16. Five samples of base flow, collected between 1983 and 1985, consisted almost entirely of wastewater effluent and were excluded from the analyses.

Another possible effect on the quality of water in the streams is the leakage of some sanitary sewers located near and under many of the streambeds. Leakage has been documented downstream of Barton Creek at Loop 360 (S1ade and others, 1986). Repairs also were made on sewers near Shoal Creek from 1983 to 1984 (John Parish, City of Austin, oral commun., 1986). Of the 18 sample sites in this study, 8 have substantial lengths of stream channel underlain with sanitary sewer lines. The potential effect of leaking sanitary sewers on water quality would be greatest during base-flow conditions.

Samples from sites $7,12,13$, and 16 may have been affected by construction upstream from the sites. Samples from site 8 may have been affected by the construction of a sanitary sewer under the channe1. Samples from sites $3,5,7,10,13,14,15$, and 16 may have been affected by discharge from wastewater treatment plants (table 2). Samples from site 18 may have been affected by leaking sanitary sewer lines prior to 1984.

\section{APPROACH}

The approach chosen to study the relation between the water-quality characteristics of streamflow and the degree of urbanization is as follows: (1) Estimate the percent of impervious cover and determine the development classification for the drainage basin of each sample site; (2) classify the flow at the time of sample collection into rising and falling stages of stormflow and base flow; (3) collect and compile selected water-quality data for each flow category; (4) compute statistical distributions of flow at the time of sample collection and concentrations of detectable water-quality properties and constituents for each flow category: (5) construct boxplots that represent the statistical distributions of the concentrations for each constituent; and (6) relate the statistical distributions to the development classification for detectable water-quality constituents for each flow category.

\section{Development Classification and Flow Categories}

The drainage basins of the sample sites were given one of four development classifications representing different ranges in percent of impervious cover. Impervious cover for the period of sampling was estimated using a city land-use study (City of Austin, Planning Department, 1982), discussions with 
Table 2.--Permitted discharge and permitted quality of effluent for wastewater facilities - within drainage basins of sample sites 1

$\left[\mathrm{ft}^{3} / \mathrm{s}\right.$, cubic feet per second; $1 \mathrm{~b} / \mathrm{d}$, pounds per day;

ND, nondischarge; --, not determined; $D$, discharge

\begin{tabular}{|c|c|c|c|c|c|c|}
\hline Wastewater facility & $\begin{array}{l}\text { Type } \\
\text { of } \\
\text { permit }\end{array}$ & $\begin{array}{l}\text { Daily } \\
\text { average } \\
\text { outflow } \\
(\mathrm{ft} / \mathrm{s})\end{array}$ & $\begin{array}{l}\text { Daily } \\
\text { average } \\
\text { biochemical } \\
\text { oxygen } \\
\text { demand } \\
(1 \mathrm{~b} / \mathrm{d}) \\
\end{array}$ & $\begin{array}{l}\text { Daily } \\
\text { average } \\
\text { total } \\
\text { suspended } \\
\text { solids } \\
(1 \mathrm{~b} / \mathrm{d})\end{array}$ & $\begin{array}{l}\text { Beginning } \\
\text { operation } \\
\text { date }\end{array}$ & $\begin{array}{l}\text { Down- } \\
\text { stream } \\
\text { sample } \\
\text { site } \\
\text { number }\end{array}$ \\
\hline Texas Tumbleweed of Aust in & ND & 0.00640 & - & - & before 1974 & 3 \\
\hline Catholic High School & ND & .0210 & 10 & -- & 1984 & 5,7 \\
\hline Lost Creek Municipal Utility District & ND & .827 & 10 & -- & 1983 & 5,7 \\
\hline Travis County Utility District, Inc. & ND & .358 & 20 & -- & 1980 & 7 \\
\hline $\begin{array}{l}\text { Shady Hollow - Southwest Travis } \\
\text { County Municipal Utility District }\end{array}$ & D & .636 & 10 & 15 & 1981 & 10 \\
\hline Country Air Mobile Home Park & D & .0570 & 10 & 15 & before 1974 & 13,16 \\
\hline Valley View Acres & D & .140 & 10 & 15 & before 1974 & 13,16 \\
\hline Lamplight Village Plant & D & .636 & 10 & 15 & before 1974 & 14,15 \\
\hline Chimneyhill Plant & D & .239 & 10 & 15 & before 1974 & 15 \\
\hline Williamson Creek Treatment Plant & D & 7.00 & 10 & 15 & before 1974 & 16 \\
\hline
\end{tabular}

1 Source: Texas Water Commission, written commun., 1987.

2 Site numbers shown in figure 2 and table 1. 
the Watershed Management Division staff of the city of Austin, and a 1981 aerial photograph. The relation between drainage area and percent of impervious cover in the basin upstream from sample sites is shown in figure 3 .

Sample sites 1 and 2 are downstream from drainage basins classified as "rural" (less than 1-percent impervious cover). Sample sites 3 to 9 are downstream from drainage basins classified as "mostly rural" (2- to 7-percent impervious cover). Sample sites 10 to 16 are downstream from drainage basins classified as "partly urban" (9- to 20-percent impervious cover). Sample sites 17 and 18 are downstream from drainage basins classified as "urban" (40percent or greater impervious cover).

The data were compiled for each site for three flow categories--rising stage of stormflow, falling stage of stormflow, and base flow. Because of the short duration of the rising stage for many storms, few data are available for this flow category except at the sites with automatic samplers (table 1). Analyses for inorganic trace elements and synthetic organic compounds from all flow categories were combined for interpretation because of the limited number of analyses with concentrations greater than the detection threshold.

\section{Data Collection and Analysis}

The data available for analysis were collected from 1974 to 1985. During data collection, several adjustments to the sampling program were made because of expanded interests, limitations of financial resources, streamgaging difficulties, and preliminary findings. Independent of the sampling program, the number of samples collected in a given year was partly determined by the number of storms. Furthermore, the distribution of samples among the sites and the three flow categories are not ideal because of the short duration of the rising stage of stormflow, concurrent flooding at numerous sites, travel time to sites, time-consuming manual sampling techniques, inclement weather, and limited personnel. Consequently, more samples were collected during the falling stage of stormflow and during base flow. Automatic samplers were installed at six sites to improve sampling during the rising stage of stormflow. At these sites, as many as eight samples could be collected at predetermined time intervals after a given stage had been exceeded. These sites are identified in figure 2 and table 1.

The frequency of sampling and the group of constituents selected for analysis are related to the assumed importance of the constituent in determining the effect of urbanization, laboratory costs, and volume of water available from the six automatic samplers. As a result, the laboratory analyses commonly included physical organics and inorganics, nutrients, and indicator bacteria. Less than one-half of all the samples collected were analyzed for inorganic trace elements and synthetic organic compounds. The sampling and analysis program for physical organics and inorganics, nutrients, indicator bacteria, and inorganic trace elements was essentially unchanged during the period of data collection. The sampling and analys is program for the synthetic organic compounds was adjusted in 1982, when analyses for some groups of compounds were discont inued and others were added. The sampling and analyses for radiochemical constituents were made only once, in 1980 . The stream discharge and the time of collection were determined and documented for each sample. 


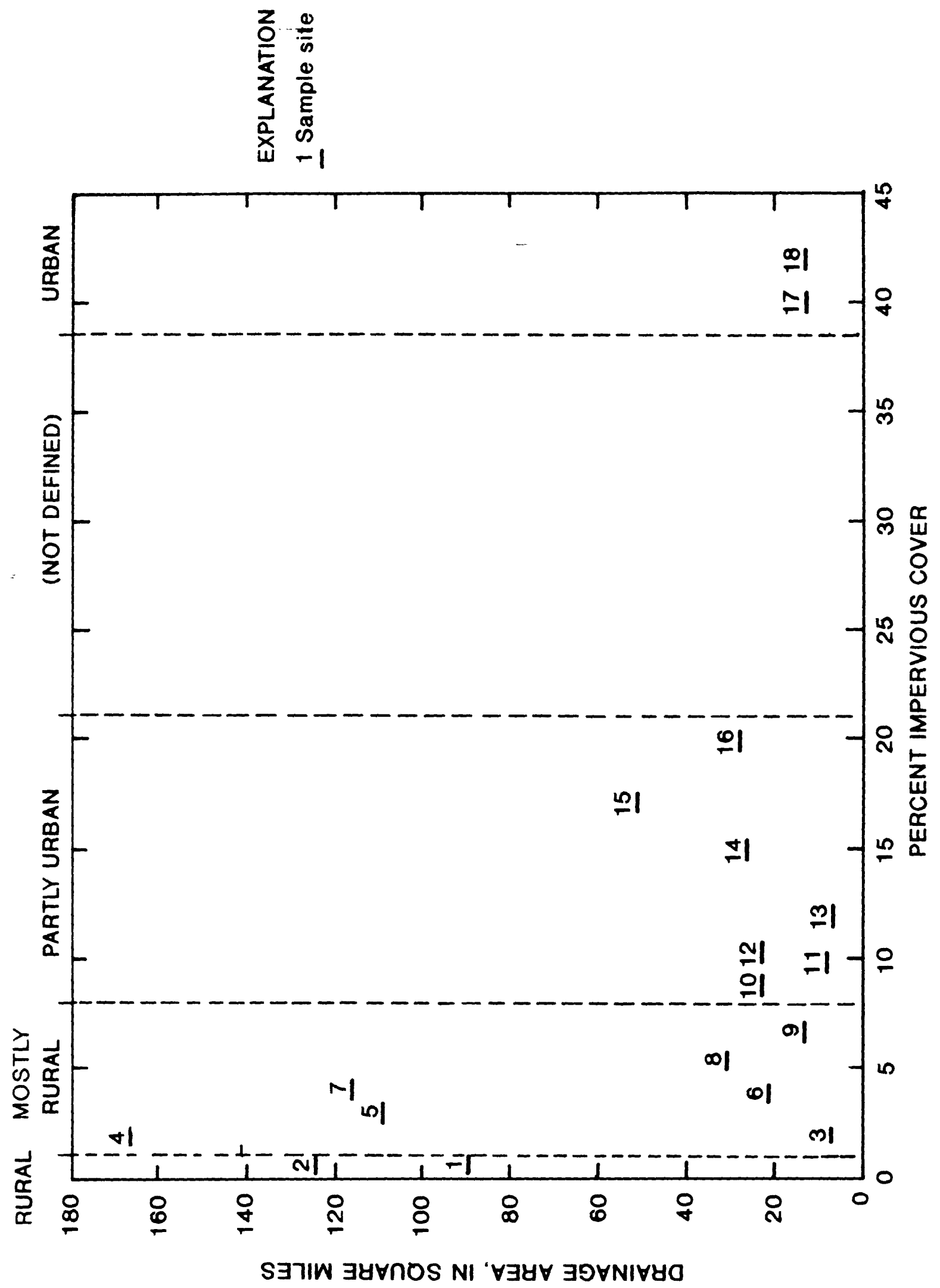

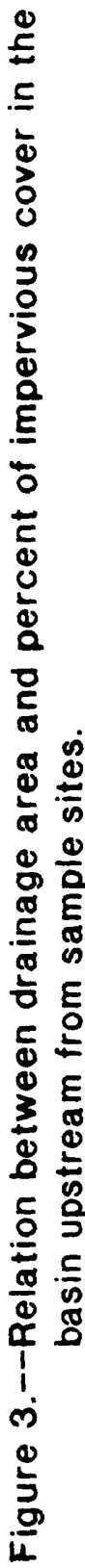




\section{Statistical Comparisons}

Statistical comparisons were used to determine whether samples collected during different flow categories at the same sample site, and from different sample sites or groups of sites with similar development classifications, were from the same or from different parent populations. The parent population at each site would be all possible samples of water quality that could be collected. Hydrologic data, including discharge and water-quality properties and constituents, usually are not normally distributed. Water-quality data tend to conform approximately to a log-normal distribution. In this study, non-parametric statistical tests were chosen to compare median concentrations from different flow categories at a given sample site and median concentrations from the same flow categories for the different development classifications. The median concentration is a good estimator of the central tendency of water-quality concentrations.

The Mann-Whitney test was used to determine whether the medians from two independent samples are from the same population with a selected confidence interval. The Mann-Whitney test uses the relative ranks of the data for comparisons and generally is considered to be distribution-free. This test was chosen for two-sample group comparisons because the Mann-Whitney test does not require that the data be normally distributed. The Mann-Whitney test was conducted on the logarithms of the concentrations; thus, the assumption of equal variance was more closely met for this two-sample comparison. The MannWhitney test is about 95 percent as accurate as the two-sample $t$ test when either test is applicable and, in fact, may be very much more powerful if the normality or sample independence assumption of the two-sample $t$ test is seriously violated. Most of the inorganic trace elements and synthetic organic compounds did not have enough analyses with detectable concentrations for adequate statistical comparison between the distributions. Therefore, a chi. square test was used to compare the proportion of samples with concentrations greater than the largest detection threshold from each of the groups.

The data were summarized statistically to show the maximum, minimum, mean, and median concentrations of each constituent for each flow category, but only the median concentrations were tabulated. In addition, several statistical quartiles were calculated as well as the interquartile range for use in the graphical comparisons of the concentrations.

Boxplots were constructed to show comparisons among flow categories and comparisons among drainage basins with different development classifications. The drawing and interpretation of boxplots are described by Tukey $(1977, p$. 27-49) and by Velleman and Hoagl in (1981, p. 65-70). The box covers the middle 50 percent of the sample population. Seventy-five percent of the concentrations are less than the upper quartile and 25 percent are less than the lower quartile. The line in the middle of the box represents the median value. This middle 50 percent of the population is the interquartile range, a good estimator of variability of data that may not be normally distributed. The upper "whisker" is drawn to the nearest value that is less than or equal to the upper quartile (top of the box) plus 1.5 times the interquartile range (box length). The lower "whisker" is drawn to the nearest value that is greater than or equal to the lower quartile (bottom of the box) minus 1.5 times the interquartile range. Values beyond these ranges are plotted individually. 
Boxplots representing the distribution of concentrations of most waterquality constituents typically have several individual outlier points that plot above the box and represent the large concentrations, and comparatively few points that plot below the box and represent small concentrations because smaller concentrations are constrained by laboratory detection thresholds or zero concentrations. Boxplots for the rising stage and falling stage of stormf low and for base flow are plotted on a logarithmic scale in figures 4 , $6,8-12,16-18,23$, and $25-29$ because of the large ranges of values in the samples collected.

A minimum of seven samples of the rising stage of stormflow and of the falling stage of stormflow and a minimum of five samples of base flow were used to construct boxplots representing the distributions of concentrations and densities for physical organics and inorganics, nutrients, and indicator bacteria. This minimum number was chosen after considering the variability of the concentrations for each flow category, along with the number of samples required for boxplot construction. As a result, only a few of the sites without automatic samplers had enough samples to create boxplots for a 11 stages of flow. For sites that had at least three samples from each flow category, but not enough samples for boxplots, a median value was calculated and plotted. The values and distributions indicated by the boxplots and median values are more reliable as the number of samples for each flow category increases (figs. 5-12 and table 3). Boxplots showing the distribution of the concentrations at each sample site were made for trace elements and synthetic organics having a minimum of five samples with concentrations greater than detection thresholds to indicate differences between sample sites.

The statistical distributions of concentrations were plotted by sample site in order of the increasing percent of impervious cover of the drainage basin. By grouping samples according to development classification, enough analyses were available for each constituent so that statistical comparison of water quality could be made among the four classifications of sites.

\section{DISTRIBUTION OF SAMPLE DISCHARGE}

The discharges for the three flow categories were compiled separately for each sample site because the water-quality properties and constituents were influenced by the streamflow at the time of sample collection. The median sample discharges by flow category for 873 of the 884 samples are presented by sample site in table 3 . The extreme discharges during sample collection at the 18 sites ranged from $0.01 \mathrm{ft}^{3} / \mathrm{s}$ at 3 of the sample sites to $9,130 \mathrm{ft}^{3} / \mathrm{s}$ at site 7. The number of samples used in the calculation of the medians for each flow category also is presented by sample site in table 3 . The median sample discharge indicates relative magnitudes of flow that have been sampled at each site and for each flow category.

Boxplots showing the distribution of the discharge during sample collection from the rising stage and falling stage of stormflow and from the base flow are presented in figure 4. The magnitudes of the discharge in rising stage and falling stage of stormflow and in base flow can be compared using the three sets of boxplots. The sample sites with smaller drainage areas have median base flows of about $1 \mathrm{ft}^{3} / \mathrm{s}$; stormflow for these sites is typically larger for about 1 or 2 days after a storm. For the sites with larger drainage areas, increased discharges may persist for 1 or 2 weeks after major 

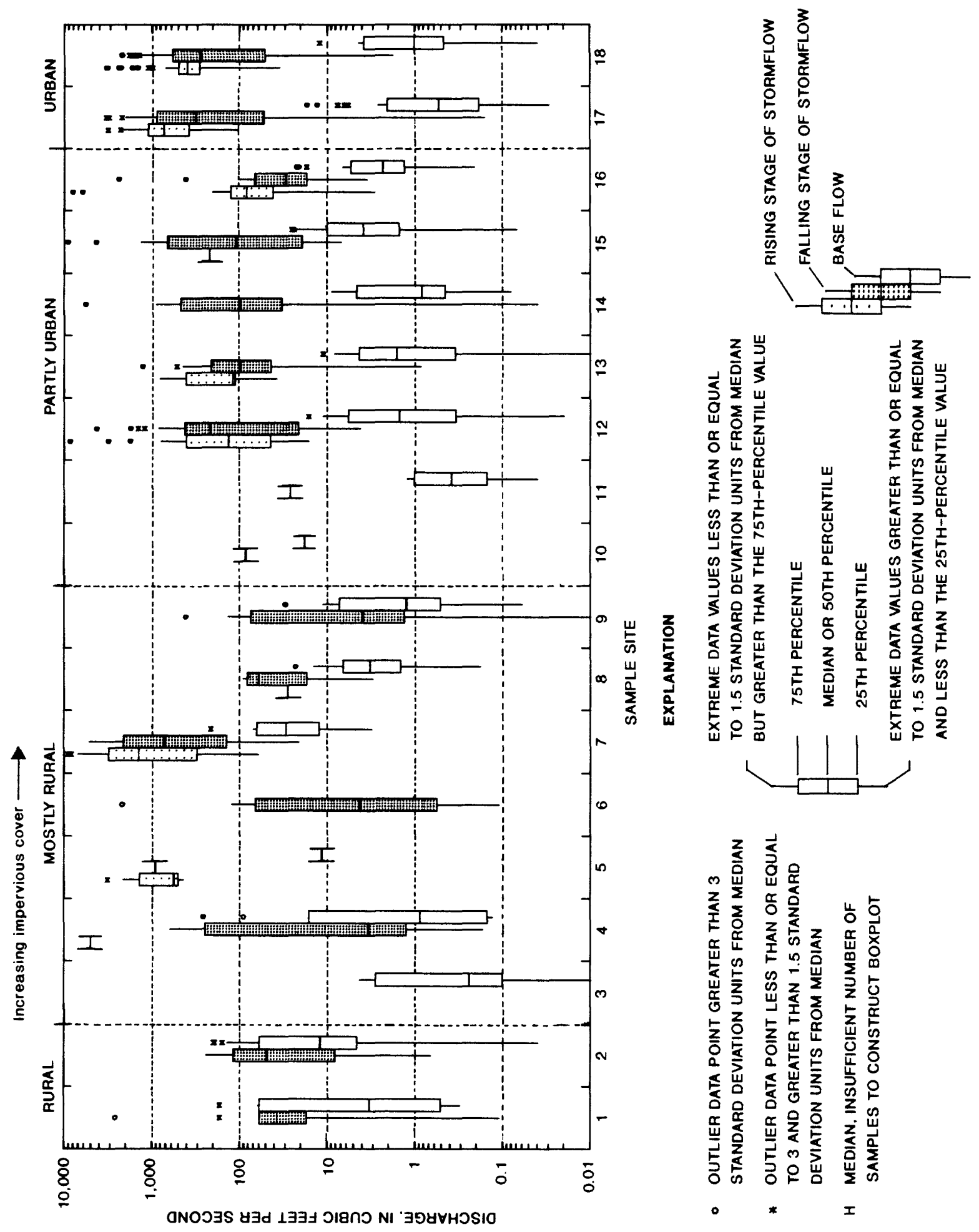

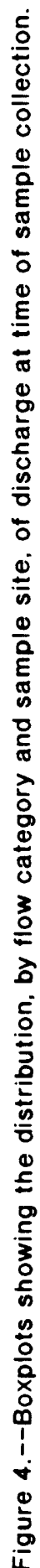


Table 3.--Median discharges and concentrations and densities of selected water-quality properties and constituents from rising stage and falling stage of

$\left[\mathrm{ft}^{3} / \mathrm{s}\right.$, cubic feet per second; mg/L, milligrams per liter: --, less than three samples:

( ), number in parentheses represents number of samples; cols./100 mL, colonies per $100 \mathrm{milliliters]}$

\begin{tabular}{|c|c|c|c|c|c|c|c|c|c|c|c|c|c|c|c|c|}
\hline \multirow{2}{*}{$\begin{array}{c}\text { Sample } \\
\text { site } \\
\text { number } \\
\text { (fig. 2) }\end{array}$} & \multirow{2}{*}{$\begin{array}{l}\text { Develop- } \\
\text { ment } \\
\text { classi- } \\
\text { fication }\end{array}$} & \multicolumn{3}{|c|}{$\begin{array}{c}\text { Discharge } \\
\left(\mathrm{ft}^{3} / \mathrm{s}\right)\end{array}$} & \multicolumn{3}{|c|}{$\begin{array}{l}\text { Dissotved } \\
\text { sol ids } \\
(\mathrm{mg} / \mathrm{L}) \\
\end{array}$} & \multicolumn{3}{|c|}{$\begin{array}{l}\text { Suspended } \\
\text { sol ids } \\
(\mathrm{mg} / \mathrm{L})\end{array}$} & \multicolumn{3}{|c|}{$\begin{array}{l}\text { Biochemical } \\
\text { oxygen demand } \\
(\mathrm{mg} / \mathrm{L})\end{array}$} & \multicolumn{3}{|c|}{$\begin{array}{l}\text { Total } \\
\text { organic carbon } \\
(\mathrm{mg} / \mathrm{L})\end{array}$} \\
\hline & & $\begin{array}{l}\text { Storm } \\
\text { Ris- } \\
\text { ing } \\
\text { stage }\end{array}$ & $\begin{array}{l}\text { flow } \\
\text { Falt- } \\
\text { ing } \\
\text { stage }\end{array}$ & $\begin{array}{l}\text { Base } \\
\text { flow }\end{array}$ & $\begin{array}{c}\text { Storm } \\
\text { Ris- } \\
\text { ing } \\
\text { stage } \\
\end{array}$ & $\begin{array}{l}\text { flow } \\
\text { FalT- } \\
\text { ing } \\
\text { stage }\end{array}$ & $\begin{array}{l}\text { Base } \\
\text { flow }\end{array}$ & $\begin{array}{l}\text { Storm } \\
\text { Ris- } \\
\text { ing } \\
\text { stage }\end{array}$ & $\begin{array}{c}\text { flow } \\
\text { Faing } \\
\text { ing } \\
\text { stage }\end{array}$ & $\begin{array}{l}\text { Base } \\
\text { flow }\end{array}$ & $\begin{array}{c}\text { Storm } \\
\text { Ris- } \\
\text { ing } \\
\text { stage }\end{array}$ & $\begin{array}{c}\text { flow } \\
\text { Fang } \\
\text { ing } \\
\text { stage }\end{array}$ & $\begin{array}{l}\text { Base } \\
\text { flow }\end{array}$ & $\begin{array}{l}\text { Storm } \\
\text { Ris- } \\
\text { ing } \\
\text { stage }\end{array}$ & $\begin{array}{l}\text { fal } \\
\text { ing } \\
\text { stage }\end{array}$ & $\begin{array}{l}\text { Base } \\
\text { flow }\end{array}$ \\
\hline 1 & Rural & -- & $\begin{array}{c}37 \\
(12)\end{array}$ & $(10)^{3.3}$ & - & $\begin{array}{l}220 \\
(11)\end{array}$ & $\begin{array}{l}230 \\
(7)\end{array}$ & -- & $\begin{array}{c}3 \\
(12)\end{array}$ & $\begin{array}{c}1 \\
(9)\end{array}$ & -- & $\begin{array}{l}0.8 \\
(12)\end{array}$ & $\begin{array}{l}0.3 \\
(9)\end{array}$ & -- & $\begin{array}{l}4.2 \\
(12)\end{array}$ & $\begin{array}{l}4.8 \\
(10)\end{array}$ \\
\hline 2 & do. & -- & $\begin{array}{c}48 \\
(14)\end{array}$ & $\begin{array}{l}12 \\
(59)\end{array}$ & -- & $\begin{array}{l}250 \\
(13)\end{array}$ & $\begin{array}{l}270 \\
(56)\end{array}$ & -- & $\begin{array}{c}8 \\
(14)\end{array}$ & $\begin{array}{c}1 \\
(58)\end{array}$ & $\cdots$ & $(14)$ & $\begin{array}{l}.5 \\
(59)\end{array}$ & - & $\begin{array}{l}3.8 \\
(14)\end{array}$ & $\begin{array}{l}2.1 \\
(55)\end{array}$ \\
\hline 3 & $\begin{array}{l}\text { Mostly } \\
\text { rural }\end{array}$ & -- & -- & $\left(\dot{9}^{24}\right.$ & - & -- & $\begin{array}{c}420 \\
(7)\end{array}$ & -- & -- & $\begin{array}{l}1 \\
(9)\end{array}$ & -- & -- & .6 & -- & -- & $\begin{array}{l}3.3 \\
(9)\end{array}$ \\
\hline 4 & do. & $\begin{array}{r}4,980 \\
(5)\end{array}$ & $(11)^{3.4}$ & $(\dot{9})$ & $\begin{array}{l}105 \\
(4)\end{array}$ & $\begin{array}{r}185 \\
(8)\end{array}$ & $\begin{array}{l}260 \\
(7)\end{array}$ & $\begin{array}{l}756 \\
(4)\end{array}$ & $\begin{array}{c}38 \\
(11)\end{array}$ & $\begin{array}{c}2 \\
(8)\end{array}$ & $\begin{array}{l}7.1 \\
(4)\end{array}$ & $(11)^{5}$ & $\begin{array}{l}.6 \\
(8)\end{array}$ & $\begin{array}{l}36 \\
(4)\end{array}$ & $\begin{array}{r}6.9 \\
(11)\end{array}$ & 2.7 \\
\hline 5 & do. & $\begin{array}{r}560 \\
(22)\end{array}$ & $\begin{array}{l}918 \\
(4)\end{array}$ & $\frac{12}{(4)}$ & $\begin{array}{l}180 \\
(3)\end{array}$ & -- & $\begin{array}{r}245 \\
(4)\end{array}$ & $\begin{array}{l}221 \\
(4)\end{array}$ & -- & $\begin{array}{c}4 \\
(4)\end{array}$ & $\begin{array}{l}1.8 \\
(8)\end{array}$ & -- & $\begin{array}{l}8 \\
(4)\end{array}$ & $\begin{array}{r}8.3 \\
(14)\end{array}$ & $\stackrel{40}{(4)}$ & $\begin{array}{r}1.7 \\
(4)\end{array}$ \\
\hline 6 & do. & -- & $\stackrel{4}{(7)}$ & -- & -- & $\begin{array}{c}181 \\
(7)\end{array}$ & -- & -- & $\begin{array}{c}126 \\
(7)\end{array}$ & -- & -- & $\begin{array}{l}3.4 \\
(7)\end{array}$ & -- & -- & $\begin{array}{l}10 \\
(6)\end{array}$ & -- \\
\hline 7 & do. & $\begin{array}{r}1,420 \\
(34)\end{array}$ & $\begin{array}{l}709 \\
(19)\end{array}$ & $\begin{array}{l}29 \\
(8)\end{array}$ & $\begin{array}{l}160 \\
(18)\end{array}$ & $\begin{array}{l}175 \\
(10)\end{array}$ & $\begin{array}{l}240 \\
(6)\end{array}$ & $\begin{array}{r}744 \\
(27)\end{array}$ & $\begin{array}{l}371 \\
(16)\end{array}$ & $\begin{array}{l}1 \\
(8)\end{array}$ & $\begin{array}{l}3.2 \\
(29)\end{array}$ & $\begin{array}{l}3.4 \\
(17)^{4}\end{array}$ & $i^{4}(8)$ & $\begin{array}{l}22 \\
(33)\end{array}$ & $\begin{array}{l}17 \\
(17)\end{array}$ & 1.7 \\
\hline 8 & do. & $\begin{array}{r}28 \\
(3)\end{array}$ & $\begin{array}{l}60 \\
(9)\end{array}$ & $\begin{array}{l}3.2 \\
(16)\end{array}$ & -- & $\begin{array}{l}230 \\
(5)\end{array}$ & $\begin{array}{l}310 \\
(7)\end{array}$ & $\begin{array}{l}207 \\
(3)\end{array}$ & $\begin{array}{c}201 \\
(9)\end{array}$ & $\begin{array}{c}13 \\
(16)\end{array}$ & $\begin{array}{l}1.6 \\
(3)\end{array}$ & $\begin{array}{l}1.2 \\
(9)\end{array}$ & $\left(i \frac{6}{16}\right)$ & $\begin{array}{l}9.1 \\
(3)\end{array}$ & $\begin{array}{l}5.2 \\
(9)\end{array}$ & $\begin{array}{l}3.6 \\
(16)\end{array}$ \\
\hline 9 & do. & -- & $(10)^{3.9}$ & $\begin{array}{l}1.2 \\
(16)\end{array}$ & -- & $\begin{array}{l}235 \\
(10)\end{array}$ & $\begin{array}{l}290 \\
(13)\end{array}$ & -- & $\begin{array}{c}34 \\
(10)\end{array}$ & $\begin{array}{c}1 \\
(16)\end{array}$ & - & $(1.5)$ & $\left(i^{4}\right)$ & -- & $\begin{array}{l}4.4 \\
(10)\end{array}$ & $\begin{array}{l}1.9 \\
(15)\end{array}$ \\
\hline 10 & $\begin{array}{l}\text { Partly } \\
\text { urban }\end{array}$ & -- & $\begin{array}{l}84 \\
(5)\end{array}$ & 18 & -- & $\begin{array}{l}179 \\
(5)\end{array}$ & -- & -- & $\begin{array}{l}174 \\
(5)\end{array}$ & $\begin{array}{c}7 \\
(3)\end{array}$ & -- & $\begin{array}{l}2.3 \\
(5)\end{array}$ & $\begin{array}{l}6 \\
(3)\end{array}$ & - & 11 & $\begin{array}{r}4.4 \\
(3)\end{array}$ \\
\hline 11 & do. & -. & $\begin{array}{l}26 \\
(3)\end{array}$ & $\dot{(5)}^{38}$ & -- & $\begin{array}{c}120 \\
(3)\end{array}$ & $\begin{array}{r}410 \\
(5)\end{array}$ & -- & $\begin{array}{c}133 \\
(3)\end{array}$ & $\begin{array}{c}4 \\
(5)\end{array}$ & -- & $\begin{array}{l}2.5 \\
(3)\end{array}$ & $\begin{array}{l}5 \\
(5)\end{array}$ & -- & $\begin{array}{l}6.7 \\
(3)\end{array}$ & $\begin{array}{r}1.4 \\
(5)\end{array}$ \\
\hline 12 & do. & $\begin{array}{r}130 \\
(28)\end{array}$ & $\begin{array}{l}210 \\
(26)\end{array}$ & $(17)^{1.5}$ & $\begin{array}{l}220 \\
\text { (17) }\end{array}$ & $\begin{array}{l}230 \\
(19)\end{array}$ & $\begin{array}{l}365 \\
(14)\end{array}$ & $\begin{array}{r}1,640 \\
(22)\end{array}$ & $\begin{array}{l}370 \\
(25)\end{array}$ & $\stackrel{2}{(17)}$ & $\begin{array}{l}5.8 \\
(24)\end{array}$ & $\begin{array}{l}3.1 \\
(26)\end{array}$ & $\left(i^{6}\right)$ & $\begin{array}{l}40 \\
(24)\end{array}$ & $\begin{array}{l}14 \\
(26)\end{array}$ & $\begin{array}{l}2.6 \\
(17)\end{array}$ \\
\hline 13 & do. & $\begin{array}{r}120 \\
(26)\end{array}$ & $\begin{array}{c}98 \\
(37)\end{array}$ & $\begin{array}{l}1.6 \\
(36)\end{array}$ & $\begin{array}{l}98 \\
(4)\end{array}$ & $\begin{array}{l}185 \\
(18)\end{array}$ & $\begin{array}{l}360 \\
(35)\end{array}$ & $\begin{array}{r}2,160 \\
(10)\end{array}$ & $\begin{array}{l}292 \\
(21)\end{array}$ & $\left(\begin{array}{c}1 \\
(35)\end{array}\right.$ & $\begin{array}{l}12 \\
(12)\end{array}$ & $\begin{array}{r}5.4 \\
(28)\end{array}$ & $(\dot{6})$ & $\begin{array}{l}36 \\
(18)\end{array}$ & $\begin{array}{l}21 \\
(29)\end{array}$ & $\begin{array}{l}3.0 \\
(33)\end{array}$ \\
\hline 14 & do. & -- & $\begin{array}{c}99 \\
(11)\end{array}$ & $\left(\dot{7}^{83}\right.$ & -- & $\begin{array}{l}160 \\
(11)\end{array}$ & $\begin{array}{c}380 \\
(5)\end{array}$ & -- & $\begin{array}{l}400 \\
(11)\end{array}$ & $\begin{array}{c}3 \\
(5)\end{array}$ & - & $\begin{array}{l}5.6 \\
\text { (11) }\end{array}$ & (7) & -- & $\begin{array}{l}19 \\
(11)\end{array}$ & $\begin{array}{r}4.8 \\
(7)\end{array}$ \\
\hline 15 & do. & $\begin{array}{l}220 \\
(6)\end{array}$ & $\begin{array}{l}110 \\
(19)\end{array}$ & $\begin{array}{r}3.8 \\
(26)\end{array}$ & $\begin{array}{c}180 \\
(6)\end{array}$ & $\begin{array}{l}165 \\
(14)\end{array}$ & $\begin{array}{l}335 \\
(16)\end{array}$ & $\begin{array}{r}1,500 \\
(6)\end{array}$ & $\begin{array}{l}536 \\
(18)\end{array}$ & $\begin{array}{c}2 \\
(22)\end{array}$ & $\begin{array}{l}7.8 \\
(6)\end{array}$ & $\begin{array}{c}3.8 \\
(18)\end{array}$ & $\begin{array}{c}7 \\
(23)\end{array}$ & $\begin{array}{l}24 \\
(6)\end{array}$ & $\begin{array}{l}13 \\
(17)\end{array}$ & $\begin{array}{l}3.4 \\
(23)\end{array}$ \\
\hline 16 & do. & $\begin{array}{r}82 \\
(13)\end{array}$ & $\begin{array}{c}29 \\
(16)\end{array}$ & $\begin{array}{r}2.3 \\
(30)\end{array}$ & $\begin{array}{c}195 \\
(8)\end{array}$ & $\begin{array}{l}160 \\
(13)\end{array}$ & $\begin{array}{l}400 \\
(16)\end{array}$ & $\begin{array}{r}180 \\
(13)\end{array}$ & $\begin{array}{l}229 \\
(16)\end{array}$ & $\begin{array}{c}6 \\
(30)\end{array}$ & $\begin{array}{c}6.2 \\
(13)\end{array}$ & $\begin{array}{r}3.4 \\
(16)\end{array}$ & $\begin{array}{l}1.6 \\
(30)\end{array}$ & $\begin{array}{l}11 \\
(13)\end{array}$ & $\begin{array}{l}11 \\
(16)\end{array}$ & $\begin{array}{l}4.4 \\
(30)\end{array}$ \\
\hline 17 & Urban & $\begin{array}{r}730 \\
(62)\end{array}$ & $\begin{array}{l}320 \\
(53)\end{array}$ & $(32)^{53}$ & $\begin{array}{l}130 \\
(11)\end{array}$ & $\begin{array}{l}150 \\
(15)\end{array}$ & $\begin{array}{l}380 \\
\text { (19) }\end{array}$ & $\begin{array}{r}2,740 \\
(20)\end{array}$ & $\begin{array}{l}552 \\
(32)\end{array}$ & $\begin{array}{c}5 \\
(32)\end{array}$ & $\begin{array}{l}17 \\
(44)\end{array}$ & $\begin{array}{r}6.6 \\
(46)\end{array}$ & $(\dot{6})$ & $\begin{array}{l}46 \\
(46)\end{array}$ & $\begin{array}{l}22 \\
(36)\end{array}$ & $\begin{array}{l}4.0 \\
(31)\end{array}$ \\
\hline 18 & do. & $\begin{array}{r}390 \\
(55)\end{array}$ & $\begin{array}{l}280 \\
(60)\end{array}$ & $\begin{array}{l}1.0 \\
(16)\end{array}$ & $\begin{array}{l}155 \\
(12)\end{array}$ & $\begin{array}{l}130 \\
(27)\end{array}$ & $\begin{array}{c}385 \\
(8)\end{array}$ & $\begin{array}{r}1,260 \\
(24)\end{array}$ & $\begin{array}{l}379 \\
(38)\end{array}$ & $\begin{array}{c}2 \\
(16)\end{array}$ & $\begin{array}{l}13 \\
(39)\end{array}$ & $\begin{array}{r}5.9 \\
(45)\end{array}$ & $\left(\begin{array}{l}8 \\
16)\end{array}\right.$ & $\begin{array}{l}32 \\
(45)\end{array}$ & $\begin{array}{l}15 \\
(43)\end{array}$ & $\begin{array}{l}3.6 \\
(15)\end{array}$ \\
\hline
\end{tabular}


Table 3.--Median discharges and concentrations and densities of selected water-quality properties and constituents from rising stage and falling stage of stormf Tow and from base flow at sample sites--Continued

\begin{tabular}{|c|c|c|c|c|c|c|c|c|c|c|c|c|c|}
\hline \multirow{3}{*}{$\begin{array}{l}\text { Sample } \\
\text { site } \\
\text { number } \\
\text { (fig. 2) }\end{array}$} & \multirow{3}{*}{$\begin{array}{c}\text { Develop- } \\
\text { ment } \\
\text { classi- } \\
\text { fication }\end{array}$} & \multicolumn{3}{|c|}{$\begin{array}{c}\text { Totat } \\
\text { nitrogen } \\
(\mathrm{mg} / \mathrm{L})\end{array}$} & \multicolumn{3}{|c|}{$\begin{array}{c}\text { Total } \\
\text { phosphorus } \\
(\mathrm{mg} / \mathrm{L})\end{array}$} & \multicolumn{3}{|c|}{$\begin{array}{c}\text { Fecal } \\
\text { coliforms } \\
(\operatorname{cols} . / 100 \mathrm{~mL}) \\
\end{array}$} & \multicolumn{3}{|c|}{$\begin{array}{c}\text { Fecat } \\
\text { streptococci } \\
(\text { cols. }(100 \mathrm{~mL}) \\
\end{array}$} \\
\hline & & Sto & if Tow & Base & Stor & flow & Base & Storn & low & Base & & & B \\
\hline & & $\begin{array}{l}\text { Ris- } \\
\text { ing } \\
\text { stage }\end{array}$ & $\begin{array}{l}\text { Fall- } \\
\text { ing } \\
\text { stage }\end{array}$ & flow & $\begin{array}{l}\text { Ris- } \\
\text { ing } \\
\text { stage }\end{array}$ & $\begin{array}{l}\text { Fatl- } \\
\text { ing } \\
\text { stage }\end{array}$ & flow & $\begin{array}{l}\text { Ris- } \\
\text { ing } \\
\text { stage }\end{array}$ & $\begin{array}{c}\text { Fant- } \\
\text { ing } \\
\text { stage }\end{array}$ & flow & $\begin{array}{l}\text { Ris- } \\
\text { ing } \\
\text { stage }\end{array}$ & $\begin{array}{c}\text { FalT- } \\
\text { ing } \\
\text { stage }\end{array}$ & f \\
\hline 1 & Rural & -- & $\begin{array}{r}0.440 \\
(12)\end{array}$ & $\begin{array}{r}0.270 \\
(10)\end{array}$ & -- & $\begin{array}{r}0.015 \\
(12)\end{array}$ & $\begin{array}{r}0.010 \\
(10)\end{array}$ & -- & $\begin{array}{r}340 \\
(12)\end{array}$ & $\begin{array}{r}22 \\
(10)\end{array}$ & -- & $\begin{array}{r}540 \\
(12)\end{array}$ & ( \\
\hline 2 & do. & -- & $\begin{array}{l}560 \\
(14)\end{array}$ & $\begin{array}{l}.310 \\
(57)\end{array}$ & -- & $\begin{array}{l}.020 \\
(14)\end{array}$ & $\begin{array}{l}.010 \\
(59)\end{array}$ & -- & $\begin{array}{r}2,900 \\
(8)\end{array}$ & $\begin{array}{r}74 \\
(20)\end{array}$ & - & $\begin{array}{r}4,750 \\
(8)\end{array}$ & ( \\
\hline 3 & $\begin{array}{l}\text { Most ly } \\
\text { rural }\end{array}$ & -- & -- & $\begin{array}{r}.385 \\
(8)\end{array}$ & -- & -- & $\begin{array}{r}.010 \\
(9)\end{array}$ & -- & -- & $\begin{array}{l}220 \\
(9)\end{array}$ & -- & -- & \\
\hline 4 & do. & $\begin{array}{r}2.95 \\
(4)\end{array}$ & $\begin{array}{c}1.08 \\
(11)\end{array}$ & $\begin{array}{r}.440 \\
(7)\end{array}$ & $\begin{array}{r}0.270 \\
(4)\end{array}$ & $\begin{array}{l}.040 \\
(11)\end{array}$ & $\begin{array}{r}.010 \\
(9)\end{array}$ & $\begin{array}{r}40,500 \\
(4)\end{array}$ & $\begin{array}{r}2,200 \\
(11)\end{array}$ & $\begin{array}{r}25 \\
(9)\end{array}$ & $\begin{array}{r}72,000 \\
(4)\end{array}$ & $\begin{array}{r}3,100 \\
(11)\end{array}$ & \\
\hline 5 & do. & $\begin{array}{l}1.10 \\
(15)\end{array}$ & $\begin{array}{l}5.90 \\
(4)\end{array}$ & $\begin{array}{r}.400 \\
(4)\end{array}$ & $\begin{array}{l}100 \\
(15)\end{array}$ & $\begin{array}{r}.600 \\
(4)\end{array}$ & $\begin{array}{r}.010 \\
(4)\end{array}$ & $\begin{array}{r}15,500 \\
(10)\end{array}$ & -- & $\begin{array}{r}18 \\
(4)\end{array}$ & $\begin{array}{r}16,000 \\
(10)\end{array}$ & -- & \\
\hline 6 & do. & -- & $\begin{array}{c}1.53 \\
(7)\end{array}$ & -- & -- & $\begin{array}{r}.110 \\
(7)\end{array}$ & -- & -- & $\begin{array}{r}9,600 \\
(6)\end{array}$ & -- & -- & $\begin{array}{r}28,000 \\
(6)\end{array}$ & \\
\hline 7 & do. & $\begin{array}{l}1.85 \\
(30)\end{array}$ & $\begin{array}{l}2.13 \\
(16)\end{array}$ & $\begin{array}{r}.465 \\
(8)\end{array}$ & $\begin{array}{l}140 \\
(30)\end{array}$ & $\begin{array}{l}.090 \\
(16)\end{array}$ & $\begin{array}{r}.015 \\
(8)\end{array}$ & $\begin{array}{r}27,000 \\
(27)\end{array}$ & $\begin{array}{r}15,000 \\
(15)\end{array}$ & $\begin{array}{l}16 \\
(8)\end{array}$ & $\begin{array}{r}27,000 \\
(27)\end{array}$ & $\begin{array}{r}18,000 \\
(15)\end{array}$ & \\
\hline 8 & do. & $\begin{array}{r}1.05 \\
(3)\end{array}$ & $\begin{array}{r}960 \\
(9)\end{array}$ & $\begin{array}{l}.405 \\
(16)\end{array}$ & $\begin{array}{r}.050 \\
(3)\end{array}$ & $\begin{array}{r}.050 \\
(9)\end{array}$ & $\begin{array}{l}.015 \\
(16)\end{array}$ & $\begin{array}{l}760 \\
(3)\end{array}$ & $\begin{array}{r}1,400 \\
(9)\end{array}$ & $\begin{array}{r}25 \\
(16)\end{array}$ & $\begin{array}{r}3,900 \\
(3)\end{array}$ & $\begin{array}{r}4,700 \\
(9)\end{array}$ & 1 \\
\hline 9 & do. & -- & $\begin{array}{l}780 \\
(10)\end{array}$ & $\begin{array}{l}.520 \\
(15)\end{array}$ & -- & $\begin{array}{l}.025 \\
(10)\end{array}$ & $\begin{array}{l}.010 \\
(16)\end{array}$ & -- & $\begin{array}{r}1,900 \\
(9)\end{array}$ & $\begin{array}{l}100 \\
(16)\end{array}$ & -- & $\begin{array}{r}2,900 \\
(9)\end{array}$ & ( \\
\hline 10 & $\begin{array}{l}\text { Partiy } \\
\text { urban }\end{array}$ & -- & $\begin{array}{c}1.29 \\
(5)\end{array}$ & $\begin{array}{r}.470 \\
(3)\end{array}$ & -- & $\begin{array}{r}.070 \\
(5)\end{array}$ & $\begin{array}{r}.020 \\
(3)\end{array}$ & -- & $\begin{array}{r}13,000 \\
(5)\end{array}$ & $\begin{array}{l}260 \\
(3)\end{array}$ & -- & $\begin{array}{r}41,000 \\
(5)\end{array}$ & 2 , \\
\hline 11 & do: & -- & $\begin{array}{r}1.10 \\
(3)\end{array}$ & $\begin{array}{r}.400 \\
(5)\end{array}$ & -- & $\begin{array}{r}.110 \\
(3)\end{array}$ & $\begin{array}{r}.010 \\
(5)\end{array}$ & -- & $\begin{array}{r}34,000 \\
(3)\end{array}$ & $\begin{array}{l}180 \\
(5)\end{array}$ & -- & $\begin{array}{r}60,000 \\
(3)\end{array}$ & \\
\hline 12 & do. & $\begin{array}{l}3.55 \\
(28)\end{array}$ & $\begin{array}{c}1.85 \\
(26)\end{array}$ & $\begin{array}{l}400 \\
(17)\end{array}$ & $\begin{array}{l}.380 \\
(28)\end{array}$ & $\begin{array}{l}140 \\
(26)\end{array}$ & $\begin{array}{l}.010 \\
(17)\end{array}$ & $\begin{array}{r}23,500 \\
(22)\end{array}$ & $\begin{array}{r}12,000 \\
(23)\end{array}$ & $\begin{array}{l}120 \\
(17)\end{array}$ & $\begin{array}{r}35,000 \\
(22)\end{array}$ & $\begin{array}{r}20,000 \\
(23)\end{array}$ & 1 \\
\hline 13 & do. & $\begin{array}{l}6.00 \\
(19)\end{array}$ & $\begin{array}{r}2.50 \\
(31)\end{array}$ & $\begin{array}{l}.570 \\
(34)\end{array}$ & $\begin{array}{l}.810 \\
(19)\end{array}$ & $\begin{array}{l}390 \\
(31)\end{array}$ & $\begin{array}{l}.130 \\
(36)\end{array}$ & $\begin{array}{r}47,000 \\
(14)\end{array}$ & $\begin{array}{r}55,000 \\
(20)\end{array}$ & $\begin{array}{r}140 \\
(10)\end{array}$ & $\begin{array}{r}140,000 \\
\text { (14) }\end{array}$ & $\begin{array}{r}90,500 \\
(20)\end{array}$ & 1 \\
\hline 14 & do. & -- & $\begin{array}{l}2.65 \\
(11)\end{array}$ & $\begin{array}{r}2.00 \\
(7)\end{array}$ & -- & $\begin{array}{l}.400 \\
(11)\end{array}$ & $\begin{array}{r}.040 \\
(7)\end{array}$ & -- & $\begin{array}{r}53,000 \\
(10)\end{array}$ & $\begin{array}{l}100 \\
(7)\end{array}$ & -- & $\begin{array}{r}76,000 \\
(11)\end{array}$ & \\
\hline 15 & do. & $\begin{array}{r}2.30 \\
(6)\end{array}$ & $\begin{array}{l}2.12 \\
(18)\end{array}$ & $\begin{array}{l}.570 \\
(23)\end{array}$ & $\begin{array}{c}1.35 \\
(6)\end{array}$ & $\begin{array}{l}.200 \\
(18)\end{array}$ & $\begin{array}{l}.020 \\
(23)\end{array}$ & $\begin{array}{r}43,000 \\
(6)\end{array}$ & $\begin{array}{r}27,000 \\
(18)\end{array}$ & $\begin{array}{r}240 \\
(23)\end{array}$ & $\begin{array}{r}87,500 \\
(6)\end{array}$ & $\begin{array}{r}64,500 \\
(18)\end{array}$ & 1 \\
\hline 16 & do. & $\begin{array}{l}1.87 \\
(13)\end{array}$ & $\begin{array}{c}1.76 \\
(16)\end{array}$ & $\begin{array}{c}1.52 \\
(30)\end{array}$ & $\begin{array}{l}390 \\
\text { (13) }\end{array}$ & $\begin{array}{l}340 \\
(16)\end{array}$ & $\begin{array}{l}.030 \\
(30)\end{array}$ & $\begin{array}{r}16,000 \\
\text { (13) }\end{array}$ & $\begin{array}{r}35,000 \\
(16)\end{array}$ & $\begin{array}{r}200 \\
(30)\end{array}$ & $\begin{array}{r}35,000 \\
(13)\end{array}$ & $\begin{array}{r}46,500 \\
(16)\end{array}$ & 1 \\
\hline 17 & Urban & $\begin{array}{l}4.04 \\
(47)\end{array}$ & $\begin{array}{l}2.08 \\
(38)\end{array}$ & $\begin{array}{l}.880 \\
(31)\end{array}$ & $\begin{array}{c}1.70 \\
(47)\end{array}$ & $\begin{array}{l}.620 \\
(38)\end{array}$ & $\begin{array}{l}.040 \\
(32)\end{array}$ & $\begin{array}{r}110,000 \\
(31)\end{array}$ & $\begin{array}{r}49,000 \\
(32)\end{array}$ & $\begin{array}{l}600 \\
(32)\end{array}$ & $\begin{array}{r}215,000 \\
(30)\end{array}$ & $\begin{array}{r}77,500 \\
(32)\end{array}$ & 1 \\
\hline 18 & do. & $\begin{array}{l}4.35 \\
(44)\end{array}$ & $\begin{array}{l}2.42 \\
(48)\end{array}$ & $\begin{array}{l}.780 \\
(16)\end{array}$ & $\begin{array}{l}1.10 \\
(45)\end{array}$ & $\begin{array}{l}.440 \\
(50)\end{array}$ & $\begin{array}{l}.035 \\
(16)\end{array}$ & $\begin{array}{r}85,000 \\
(36)\end{array}$ & $\begin{array}{r}37,000 \\
(37)\end{array}$ & $\begin{array}{r}1,800 \\
(16)\end{array}$ & $\begin{array}{r}160,000 \\
(36)\end{array}$ & $\begin{array}{r}75,000 \\
(38)\end{array}$ & 1 \\
\hline
\end{tabular}


storms. Therefore, base-flow samples collected at two different sites 1 week after a given storm may have substantially different discharges.

\section{DISTRIBUTION OF WATER-QUALITY PROPERTIES AND CONSTITUENTS}

Water-quality properties and constituents were related to the drainagebasin development classification. These properties and constituents include physical organics and inorganics, nutrients, indicator bacteria, inorganic trace elements, synthetic organic compounds, and radiochemical constituents.

\section{Physical Organics and Inorganics}

The physical organics and inorganics included in this statistical analys is are dissolved solids, suspended solids, biochemical oxygen demand $(B O D)$, and total organic carbon (TOC).

\section{Dissolved Solids}

Storm runoff begins as precipitation that has dissolved-solids concentrations of less than $50 \mathrm{mg} / \mathrm{L}$ (milligrams per liter) (Hem, 1985, p. 36). Large concentrations of dissolved solids are typical of base-flow samples that have been in contact with the parent geologic material and soils in the drainage basin for relatively long periods of time. Stormflow moves through the drainage basin rather quickly compared to base flow, having less time to dissolve the parent geologic and soil material. The sites with the largest drainage areas have the smallest dissolved-solids concentrations during base flow, probably because the basins are less urban.

The concentrations of dissolved solids in this report were determined by summing the concentrations of the dissolved major constituents. These constituents are calcium, magnesium, sodium, potassium, bicarbonate or alkalinity, carbonate, sulfate, chloride, fluoride, silica, and minor or trace concentrations of other inorganic constituents.

The distribution of dissolved-solids concentrations is plotted by flow category in figure 5. The range of dissolved-solids concentrations for 513 samples was from $9 \mathrm{mg} / \mathrm{L}$ at site 6 to $730 \mathrm{mg} / \mathrm{L}$ at site 3 . There is no noticeable relation between the distribution of dissolved-solids concentrations in the rising and falling stages of stormflow and the development classification of the drainage basin at each sample site (fig. 5). However, the dissolved-solids concentrations generally are larger in base flow at sites with larger percentages of impervious cover. The median dissolvedsolids concentrations in each flow category at the 18 sample sites are presented in table 3.

Specific conductance is a measure of the ability of water to conduct an electrical current. This property is related to the types and concentrations of ions in the solution. Specific conductance is useful as a general indication of the dissolved-solids concentration or for extrapolating concentrations of major ions when comprehensive analyses are available only for some of the samples (Hem, 1970, p. 99). 

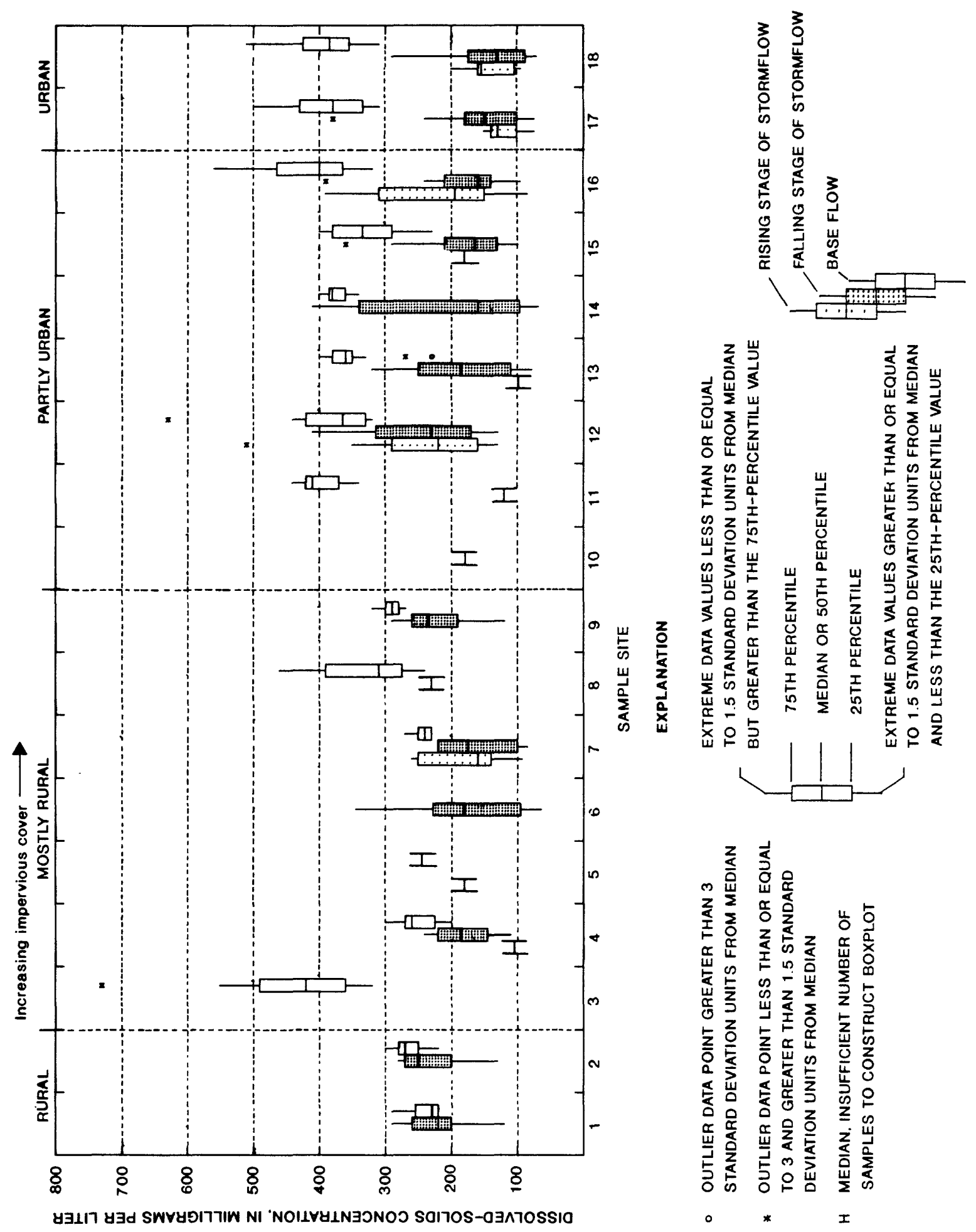

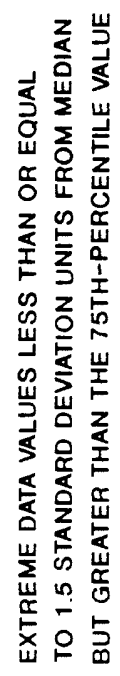

这㛏

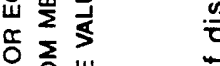

和出出

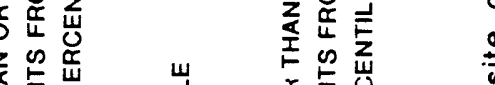

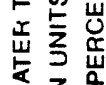

崖

嵌

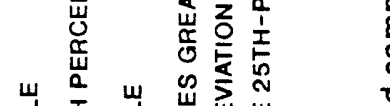

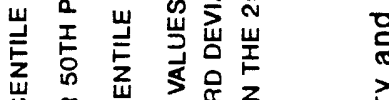

尊

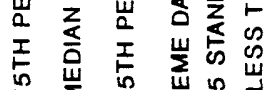

i $\frac{\omega}{2} \stackrel{n}{N}$

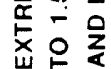

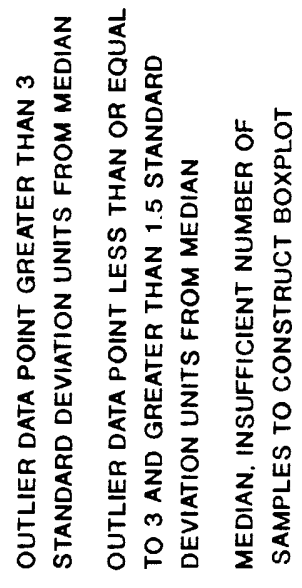

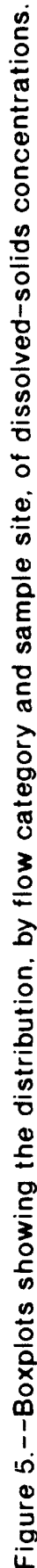


The 912 measurements of specific conductance obtained for this study ranged from $112 \mu \mathrm{S} / \mathrm{cm}$ (microsiemens per centimeter) at site 18 to $1,050 \mu \mathrm{S} / \mathrm{cm}$ at site 12. Linear-regression equations, given in table 4, predict dissolvedsolids concentration from specific-conductance values for 14 sample sites where a sufficient number of measurements of both characteristics exist to calculate a statistically significant relation. At these 14 sites, the slope of the relation is fairly similar for the regression equations, ranging from 0.533 to 0.611 . The equations are applicable for the ranges in specific conductance listed with each equation.

\section{Suspended Solids}

Suspended solids include any organic or inorganic material held in suspension by water, including silt and clay particles as well as decaying organic matter. Suspended solids generally transport nutrients, pesticides, trace elements, and other constituents that may be absorbed or adsorbed by the suspended particles. As a result, the water samples with larger suspendedsolids concentrations tend to transport more constituents. Construction upstream from a site tends to increase the suspended-solids concentration of samples.

The median concentrations of suspended solids collected at each of the 18 sites are presented by flow category in table 3 . The distribution of suspended-solids concentrations in the rising stage and falling stage of stormf low and in base flow at the 18 sites is shown in figure 6 . Suspendedsolids concentrations for 687 samples ranged from $<1 \mathrm{mg} / \mathrm{L}$ in base flow at many of the sites to $6,670 \mathrm{mg} / \mathrm{L}$ at site 18 during stormflow.

The median concentrations of suspended solids in the rising stage of stormflow were larger than those in the falling stage for each of the sites, except at site 16. The base-flow median concentrations are typically orders of magnitude less than the stormflow medians. Many water-quality constituents are associated with suspended solids; stormflow can transport larger concentrations of suspended solids than base flow. The median suspendedsolids concentrations in the rising stage of stormflow ranged from 180 to $2,740 \mathrm{mg} / \mathrm{L}$, whereas the median concentrations in the falling stage ranged from 3 to $552 \mathrm{mg} / \mathrm{L}$. Base-flow median concentrations were all less than $10 \mathrm{mg} / \mathrm{L}$ at all sites except site 8 , which had a median concentration of $13 \mathrm{mg} / \mathrm{L}$.

The concentrations of suspended solids at sites having urban and partly urban drainage basins generally were larger than those at sites having rural and mostly rural basins, except at sites 7 and 8 . The large concentrations during stormflow at these two sites may have been affected by construction upstream in the drainage basin during the period of sampling.

The drainage area of site 12 is included in the drainage area of site 8. Suspended-solids concentrations in the falling stage of stormflow at site 8 were somewhat smaller than at site 12. Less intensive development upstream from site 8 during sample collection (1975-78) than was present during sample collection (1978-85) at site 12 may have caused this phenomenon.

Suspended-solids concentrations in base flow from partly urban and urban sites were slightly larger than concentrations from rural and mostly rural sites, except at site 8. Base-flow concentrations at site 8 were larger than all other base-flow concentrations, possibly as a result of sanitary-sewer 

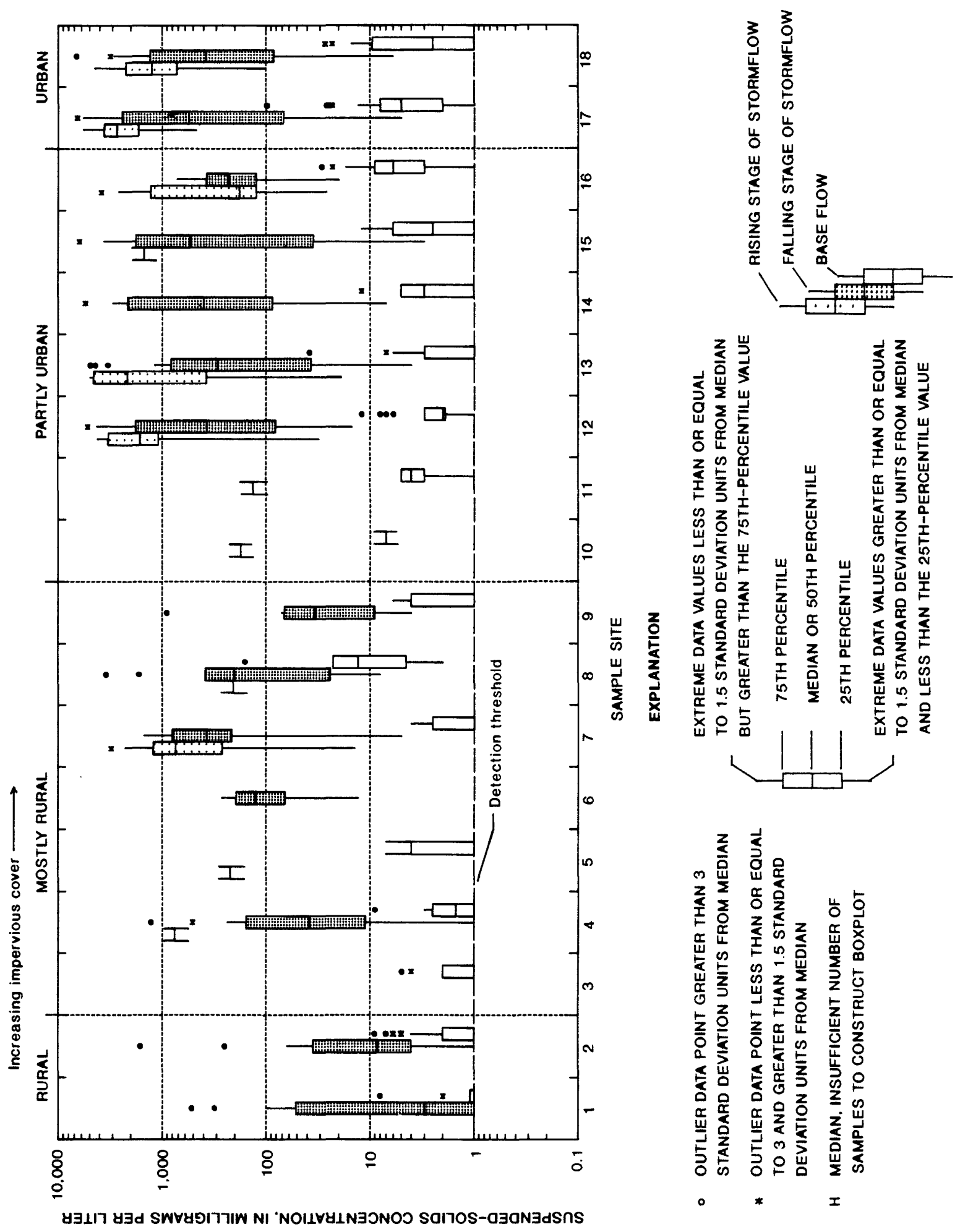

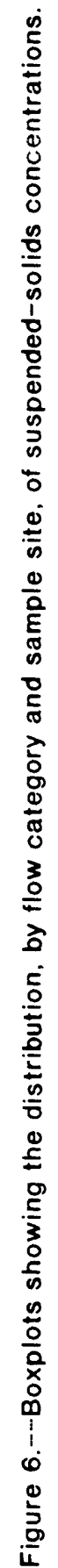


Table 4.--Relation between dissolved-solids concentration and specific conductance at selected sample sites

[K, specific-conductance value; $\mu \mathrm{S} / \mathrm{cm}$, microsiemens per centimeter at $25^{\circ}$ Celsius; $S$, dissolved-solids concentration]

\begin{tabular}{|c|c|c|c|c|c|c|}
\hline $\begin{array}{c}\text { Sample } \\
\text { site } \\
\text { number } \\
(\mathrm{fig} .2)\end{array}$ & Station name & $\begin{array}{l}\text { Number } \\
\text { of } \\
\text { samples }\end{array}$ & & $\begin{array}{l}\text { Regression } \\
\text { equation }\end{array}$ & $\begin{array}{c}\text { Correlation } \\
\text { coefficient } \\
R\end{array}$ & $\begin{array}{l}\text { Range } \\
\text { in } K \\
(\mu \mathrm{S} / \mathrm{cm})\end{array}$ \\
\hline 1 & $\begin{array}{l}\text { Barton Creek at U.S. Highway } 71 \\
\text { near Oak Hill, Tex. }\end{array}$ & 16 & $S=$ & $0.533 k+10.4$ & 0.977 & $131-501$ \\
\hline 2 & Onion Creek near Driftwood, Tex. & 69 & $S=$ & $.533 k+37.6$ & .954 & $142-584$ \\
\hline 4 & Onion Creek at Buda, Tex. & 18 & $S=$ & $.579 k-7.79$ & .992 & $115-545$ \\
\hline 7 & $\begin{array}{l}\text { Barton Creek at Loop } 360 \text {, } \\
\text { Austin, Tex. }\end{array}$ & 33 & $S=$ & $.564 k+.09$ & .988 & $141-470$ \\
\hline 8 & $\begin{array}{l}\text { Bull Creek at Farm Road } 2222 \\
\text { near Aust in, Tex. }\end{array}$ & 13 & $S=$ & $.611 k-15.9$ & .990 & $292-759$ \\
\hline 9 & $\begin{array}{l}\text { Bear Creek be low Farm Road } 1826 \\
\text { near Driftwood, Tex. }\end{array}$ & 23 & $S=$ & $.563 k-4.63$ & .974 & $137-550$ \\
\hline 12 & $\begin{array}{l}\text { Bul1 Creek at Loop } 360 \\
\text { near Aust in, Tex. }\end{array}$ & 49 & $S=$ & $.583 k-2.91$ & .990 & $196-1,050$ \\
\hline 13 & $\begin{array}{l}\text { Williamson Creek at Oak } \mathrm{Hill} \text {, } \\
\text { Tex. }\end{array}$ & 56 & $S=$ & $.578 k-4.11$ & 993 & $132-706$ \\
\hline 14 & $\begin{array}{l}\text { Walnut Creek at Dessau Road, } \\
\text { Austin, Tex. }\end{array}$ & 16 & $S=$ & $.563 k+5.14$ & .993 & $124-738$ \\
\hline 15 & $\begin{array}{l}\text { Walnut Creek at Webberville } \\
\text { Road, Austin, Tex. }\end{array}$ & 35 & $S=$ & $.568 k+2.47$ & .992 & $127-682$ \\
\hline 16 & $\begin{array}{l}\text { Williamson Creek at Jimmy Clay } \\
\text { Road, Austin, Tex. }\end{array}$ & 42 & $S=$ & $.567 k+3.18$ & .975 & $139-951$ \\
\hline 17 & $\begin{array}{l}\text { Boggy Creek at U.S. Highway 183, } \\
\text { Aust in, Tex. }\end{array}$ & 43 & $S=$ & $.586 k-1.19$ & .996 & $112-830$ \\
\hline 18 & $\begin{array}{l}\text { Shoal Creek at 12th Street, } \\
\text { Aust in, Tex. }\end{array}$ & 47 & $S=$ & $.580 k+.57$ & .992 & $117-887$ \\
\hline
\end{tabular}


construction in the channel upstream from site 8 during sampling. The suspended-solids concentration at site 16 may have been affected by upstream construction, wastewater-effluent discharge (table 2), or both.

\section{Biochemical Oxygen Demand}

Biochemical oxygen demand (BOD) is a measure of the quantity of oxygen required for the decomposition of organic matter by microorganisms, such as bacteria, present in the water sample during a 5-day period. The organic matter may originate from industrial or municipal waste or from decaying plants and animals in the drainage basin.

The median concentrations of $B O D$ in the three flow categories are shown in table 3 for the 18 sites. The BOD concentration in 770 samples ranged from 0 at four of the sites to $48 \mathrm{mg} / \mathrm{L}$ at site 17 . The distribution of BOD concentrations is presented in figure 7 .

For each site, BOD concentrations in samples collected during the rising stage were greater than those in the falling stage, except at site 7 , and the concentrations in the falling stage exceeded those for base flow. The median BOD concentration in base flow at site 16 was larger than that at the other sites, possibly as a result of discharges of wastewater from the Williamson Creek treatment plant (table 2). The concentration of BOD for the three flow categories indicates a general increase in the range of $B O D$ concentrations with increased impervious cover.

\section{Total Organic Carbon}

Total organic carbon (TOC) is a measure of the amount of organic carbon in water. Concentrations of TOC greater than about $5 \mathrm{mg} / \mathrm{L}$ in surface water may indicate sources of organic constituents from human activities.

The median concentrations of TOC in the three flow categories are presented in table 3 for each site. Concentrations of TOC in the 776 samples ranged from $0.5 \mathrm{mg} / \mathrm{L}$ in a sample collected at site 2 to $250 \mathrm{mg} / \mathrm{L}$ in a sample collected at site 18 . Concentrations of TOC were larger in the rising stage of stormflow than in the falling stage, except at site 5; rising-stage and falling-stage concentrations generally were substantially larger than baseflow concentrations at each site (fig. 8).

Many apparent inconsistencies among sites within development classifications may be explained by differences in the percent of impervious cover, particularly within the partly urban classification. For many of the less urban sites, the source of TOC probably is the natural vegetation and stream environment; the more urban sites are subject to street runoff and industrial and effluent discharges that affect TOC concentrations.

\section{Nutrients}

The two nutrients analyzed in this study were total nitrogen and total phosphorus. These nutrients were chosen because they are components in the metabolic wastes of humans and animals, and in fertilizer. Total nitrogen was chosen because the oxidation state of nitrogen changes as it passes through an 

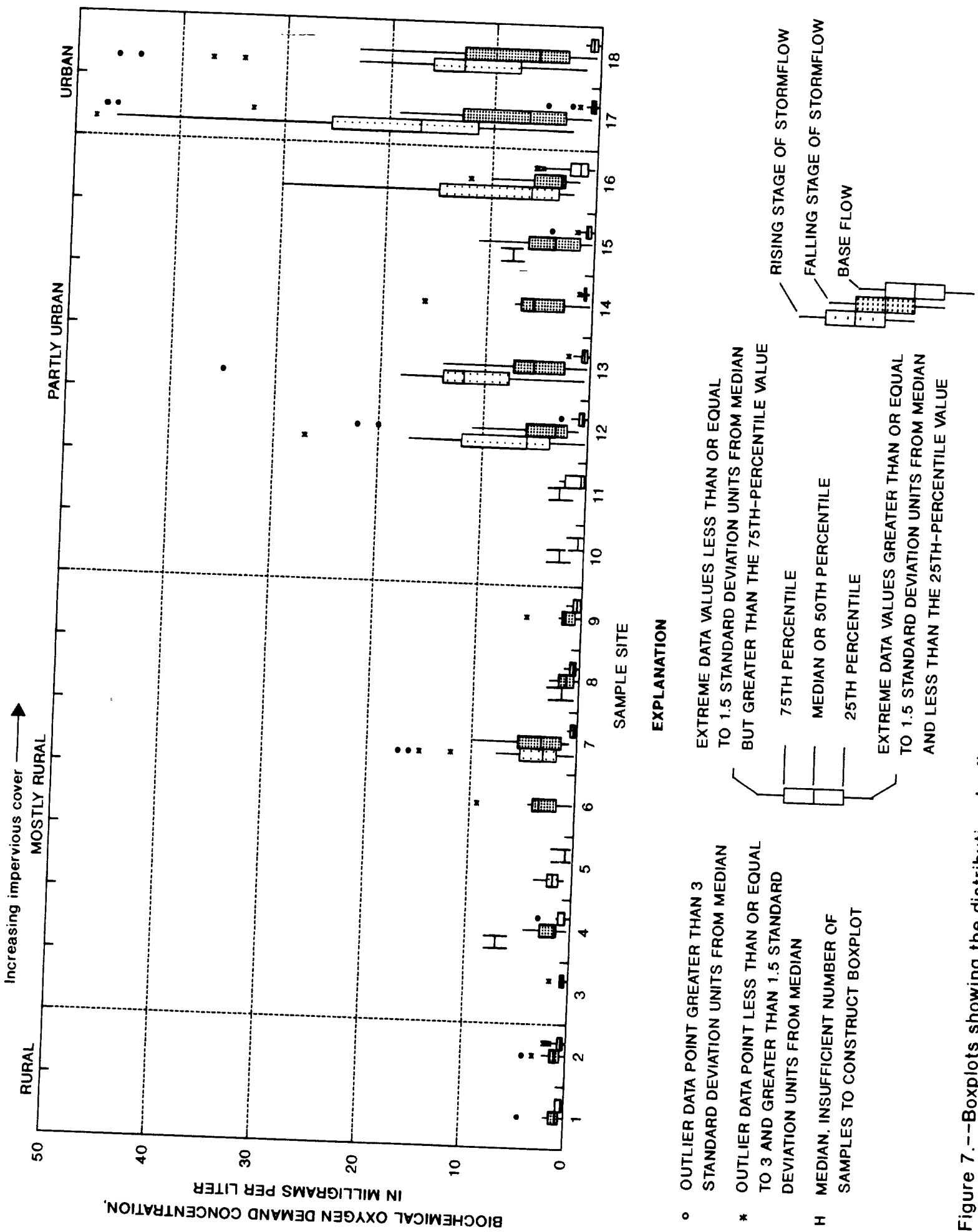

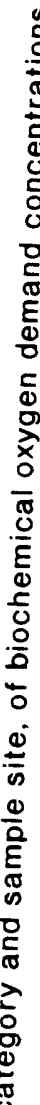

岁占焉

这동

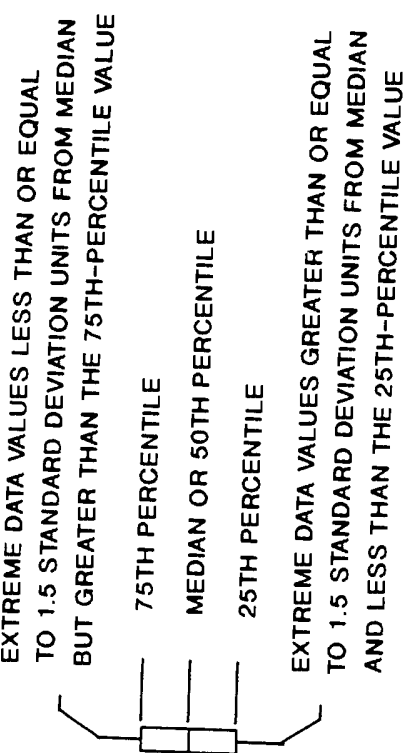

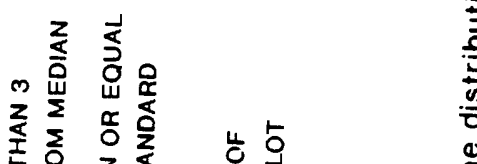

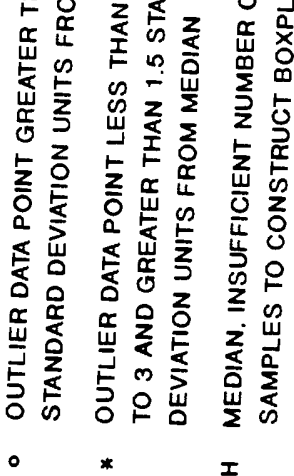

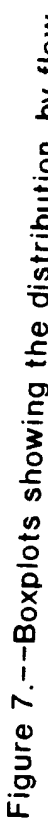




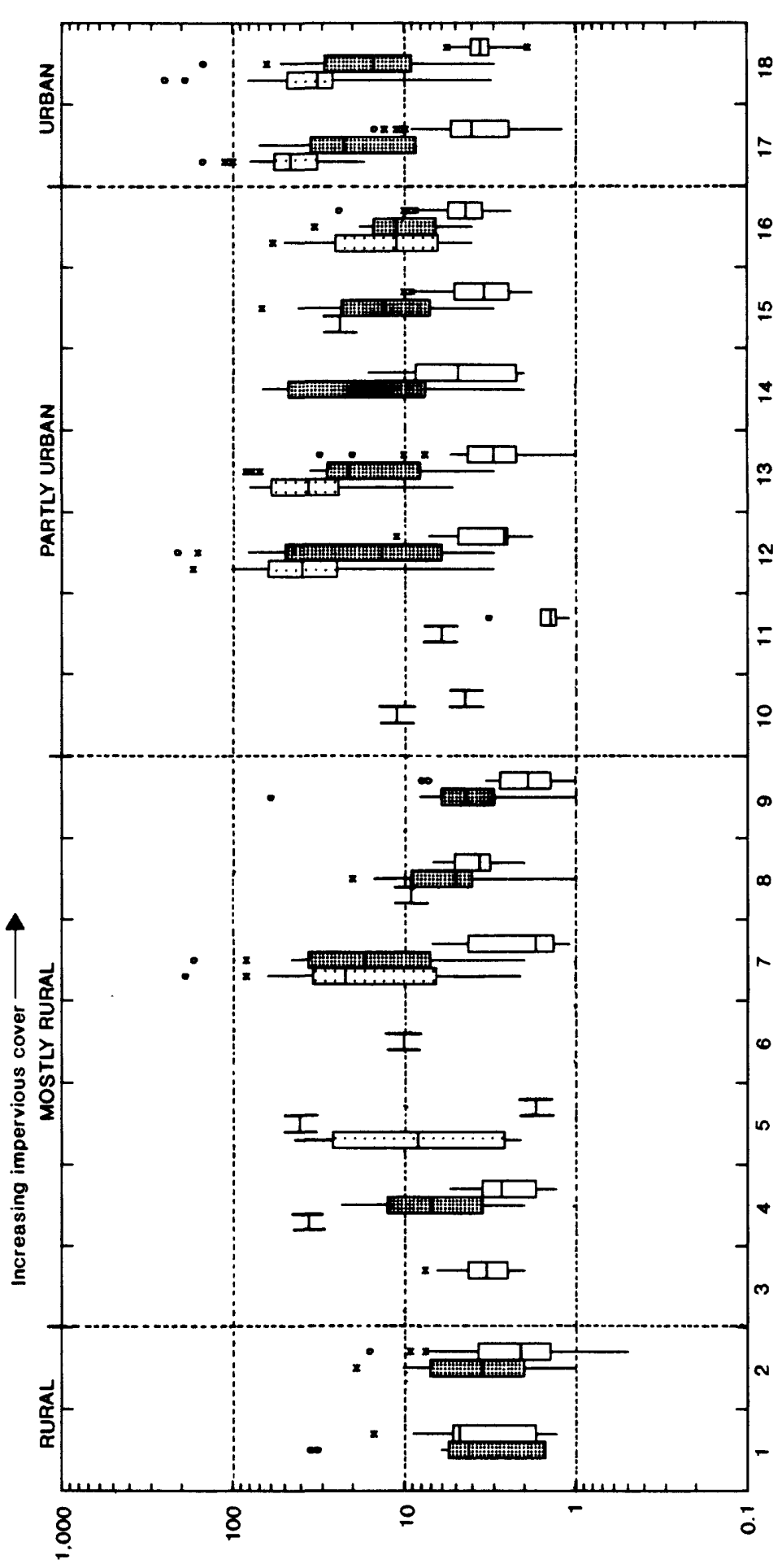

\&

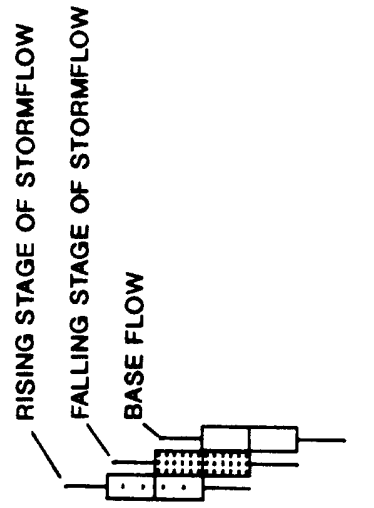

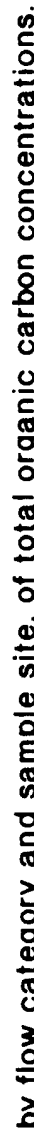

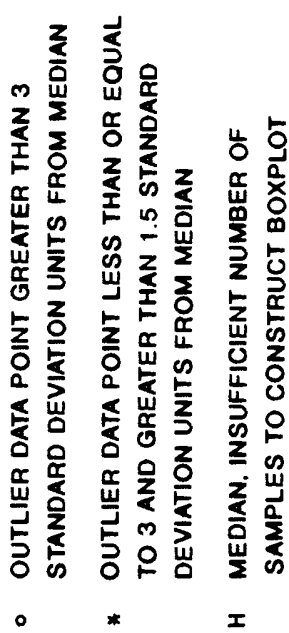

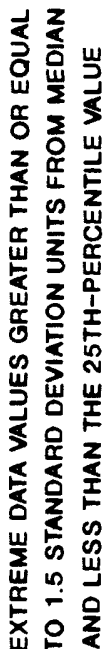
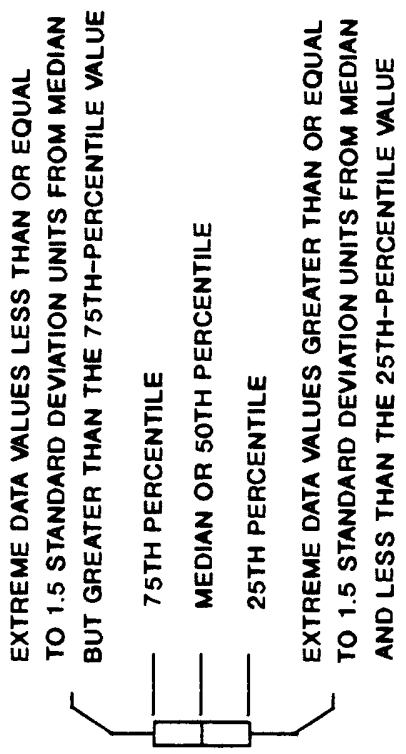

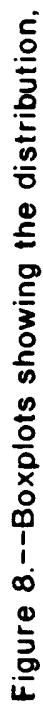


oxidizing environment. Household detergents also are an important source of phosphorus. The presence of these two nutrients may result from the sources described above, or from naturally occurring decomposition of plant and animal matter or leaching of soils and rocks.

\section{Total Nitrogen}

The median concentrations of total nitrogen in the three flow categories are presented in figure 9 and $\operatorname{table}^{-3}$ for each of the 18 sample sites. The total nitrogen concentrations in 790 samples ranged from $0.040 \mathrm{mg} / \mathrm{L}$ at site 1 to $20.0 \mathrm{mg} / \mathrm{L}$ at site 18 .

Median concentrations of total nitrogen in the rising stage were larger than those in the falling stage except at sites 5 and 7 . Median concentrations of total nitrogen were larger in both the rising stage and falling stage of stormflow than in base flow. Median base-flow concentrations were larger in samples from sites 14 and 16 than in those from the other sites, possibly as a result of wastewater-effluent discharges from the Williamson Creek treatment plant above site 16 or an upstream private treatment plant above site 14 (table 2).

Within each flow category, larger concentrations of nitrogen tend to coincide with drainage basins having larger percentages of impervious cover. Median nitrogen concentrations in the rising stage of stormf low vary directly with site development. Wastewater-effluent discharges from two facilities upstream from site 13 (table 2) may be responsible for the large nitrogen concentrations at that site.

The two sample sites with rural drainage basins and three of the sample sites with mostly rural basins had median nitrogen concentrations of less than $1.10 \mathrm{mg} / \mathrm{L}$ in the falling stage. The median concentrations in the falling stage for the remaining sites ranged from 1.10 to $2.65 \mathrm{mg} / \mathrm{L}$, with the exception of site 5 . The largest falling-stage median concentration $>5.90$ $\mathrm{mg} / \mathrm{L}$ ) is for the four samples from site 5 . Wastewater-effluent irrigation in the drainage basin for site 5 may contribute to these larger nitrogen concentrations.

Base-flow median concentrations generally increased for drainage basins with larger percentages of impervious cover. The two sites mentioned earlier (sites 14 and 16), where flow includes discharges of wastewater effluent, had the largest median concentrations.

\section{Total Phosphorus}

The median concentrations of total phosphorus in the three flow categories are presented in table 3 for the 18 sample sites. The total phosphorus concentrations in 792 samples ranged from $0.010 \mathrm{mg} / \mathrm{L}$ at several sites to $8.30 \mathrm{mg} / \mathrm{L}$ at site 17. Many analyses of $0.010 \mathrm{mg} / \mathrm{L}$ or less were reported at the sample sites downstream from rural and mostly rural drainage basins.

Distributions of total phosphorus in the rising stage and falling stage of stormflow and in base flow are shown in figure 10. Median concentrations of phosphorus are larger in the rising stage than in the falling stage, except 

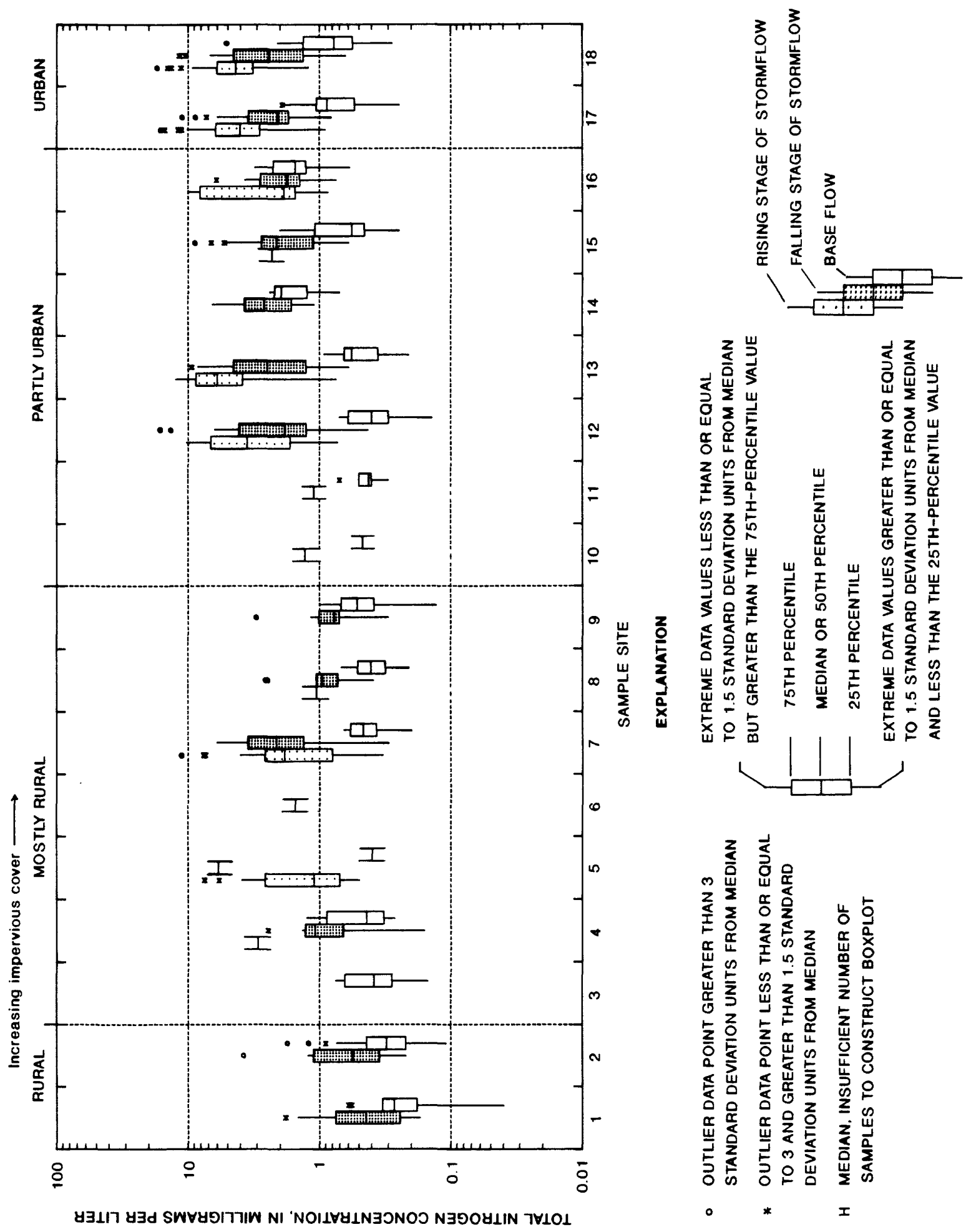

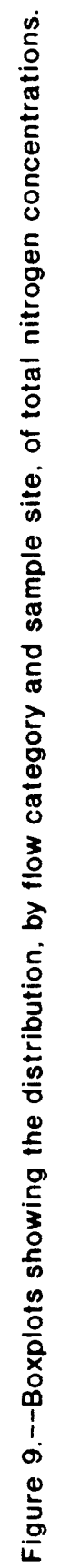



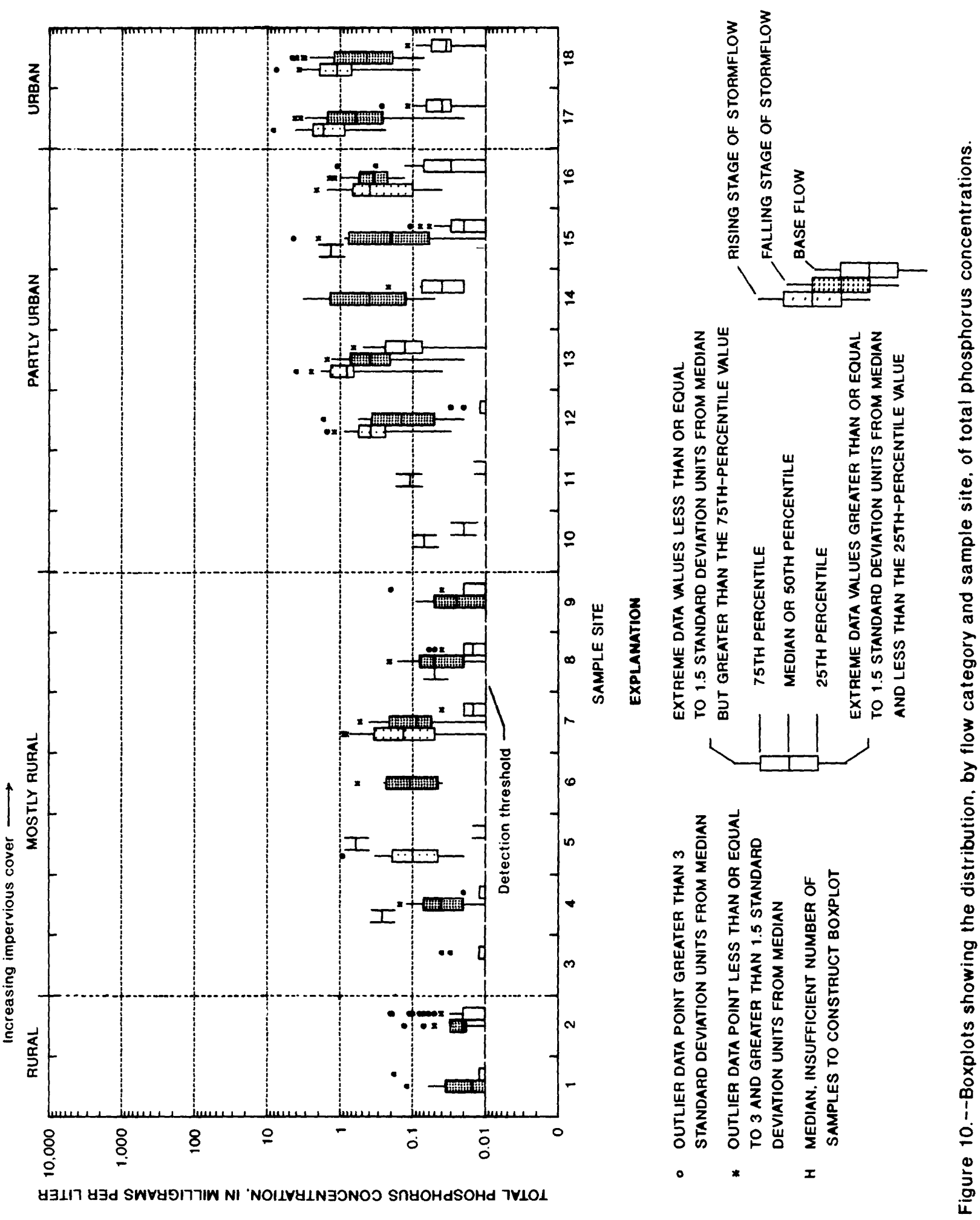
at site 5, and larger in the rising stage and falling stage than in base flow at each site.

The two sites that drain urban basins (sites 17 and 18) generally had larger median phosphorus concentrations in rising stage and falling stage than did the other sites. The only sites with median phosphorus concentrations greater than $0.020 \mathrm{mg} / \mathrm{L}$ in base flow were the two sites draining urban basins and three of the sites with partly urban basins (sites 13, 14, and 16). Sample sites 13 and 14 have substantially larger concentrations than would be expected for the development classification. New-home construction requiring fertilization of lawns (site 13) or discharge from a private treatment plant (site 14) may have caused the larger total phosphorus concentrations in base flow.

\section{Indicator Bacteria}

Samples were analyzed for two indicator bacteria--fecal coliforms and fecal streptococci. Fecal-coliform bacteria have been used as an indicator of the sanitary quality of water since the 1930 's. The bacteria are present in the intestines and feces of humans and other warm-blooded animals, and their presence in water suggests fecal contamination, which is the most likely source of pathogenic microorganisms. Fecal-streptococcal bacteria also live in the intestines of warm-blooded animals, and their presence in water is considered evidence of fecal contamination (Geidreich and Kenner, 1969, $p$. 348). One potentially valuable aid in identifying the source of bacterial contamination is the comparison of fecal-coliform densities to fecalstreptococcal densities. According to Geldreich and Kenner (1969, p. 349), the ratio of the density of fecal-coliform bacteria to that of fecalstreptococcal bacteria in the feces of man and in domestic wastewater is always greater than 4.0. The same ratio in the feces of farm animals, cats, dogs, and rodents in stormwater systems and in farmland drainage generally is less than 0.7. However, Geldreich and Kenner (1969) cautioned that the use of the ratio for distinguishing between human and animal sources would be valid only during the initial 24-hour travel time from the point of contamination because the attrition rates of the two forms of bacteria are different. In urban runoff, the sources of contamination are assumed to be non-point, and the possibility of different times of travel for water containing the two bacteria diminishes the validity of the ratio technique for determining sources.

\section{Fecal Coliforms}

The median densities of fecal coliforms collected at the sites during the three flow categories are presented in table 3 . For the 648 samples of fecal coliforms analyzed, the densities ranged from $1 \mathrm{col} . / 100 \mathrm{~mL}$ (colony per 100 milliliters) at site 8 , to $2,900,000$ cols. $/ 100 \mathrm{~mL}$ at site 18 . Larger densities in samples from site 18 possibly are caused by leaking sanitary sewers that were repaired between 1983 and 1984.

Most of the median densities of fecal coliforms in the rising stage are slightly larger than those in the falling stage at each site except sites 13 and 16 (fig. 11). Stormflow density distributions are much larger than those in base flow for each site. Within each flow category, densities of fecal 

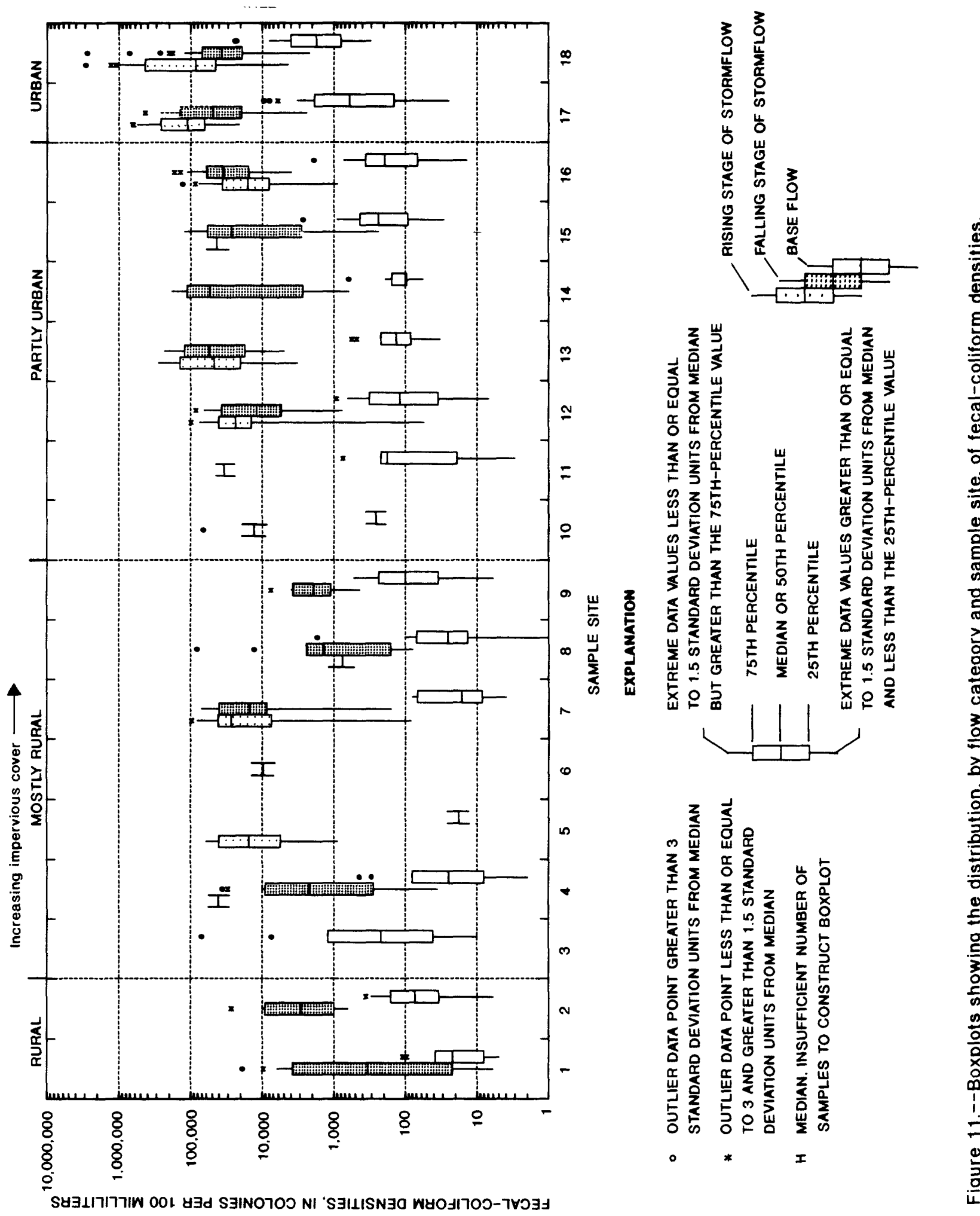

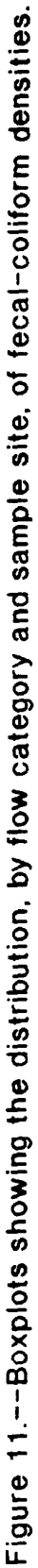


coliforms from sites with urban drainage basins generally are larger than densities from those sites with mostly rural and rural basins.

Median densities in the falling stage of 55,000 cols. $/ 100 \mathrm{~mL}$ at site 13 and 53,000 cols. $/ 100 \mathrm{~mL}$ at site 14 are slightly larger than those at the other sites with partly urban basins. Discharges from upstream wastewater-treatment plants may have some influence on the fecal-coliform densities of the samples collected at those sites.

The base-flow median density of 220 cols. $/ 100 \mathrm{~mL}$ at site 3 possibly resulted from documented effluent discharges from an upstream, private treatment plant with a non-discharge permit (table 2) (Sandra Johnson, Texas Water Commission, oral commun., 1987).

\section{Fecal Streptococci}

The median densities of fecal streptococci are presented for the sites in table 3. The 649 fecal-streptococcal samples have densities ranging from 4 cols. $/ 100 \mathrm{~mL}$ at one sample site to $1,900,000$ cols. $/ 100 \mathrm{~mL}$ at site 17 . Fecalstreptococcal densities in the rising stage and falling stage of stormflow and in base flow are shown in figure 12.

Median fecal-streptococcal densities in the rising stage of stormflow are slightly larger than those in the falling stage, except at sites 8 and 16 . For each site, densities in the rising stage and falling stage are substantially larger than those in base flow. Within each flow category, the distributions of the densities from the sites with urban drainage basins were slightly larger than the distributions of densities from sites with rural basins. However, the mostly rural sites 4 and 7 have larger fecal-streptococcal densities because there are more farm animals upstream from those sites.

\section{Ratios of Fecal Coliforms to Fecal Streptococci}

The source of bacteria based on ratios of fecal coliforms to fecal streptococci could not be conclusively determined because of unknown travel times from the source or sources. However, some patterns between the distribution of each flow category for the two bacterial densities (figs. 11 and 12) are apparent. The sites with urban basins (17 and 18) have larger densities of fecal coliforms than fecal streptococci in base flow, which indicates that at least part of the source may be human. A comparison of the bacterial densities from the sites with mostly rural drainage basins (table 3 ) indicates animal sources. The ratios at each sample site indicate mixtures of human and animal sources in the rising stage and falling stage of stormflow.

\section{Inorganic Trace Elements}

The detection of a particular inorganic trace element at a site is a function of the existence of that element in the drainage basin, the solubility of the element, and the laboratory detection threshold for the chemical analysis used. Although many trace elements are associated with sediment or suspended sediment, the concentrations reported in this analys is reflect only the dissolved fraction from samples filtered prior to analysis. The health-related concern about some of the inorganic trace elements results 

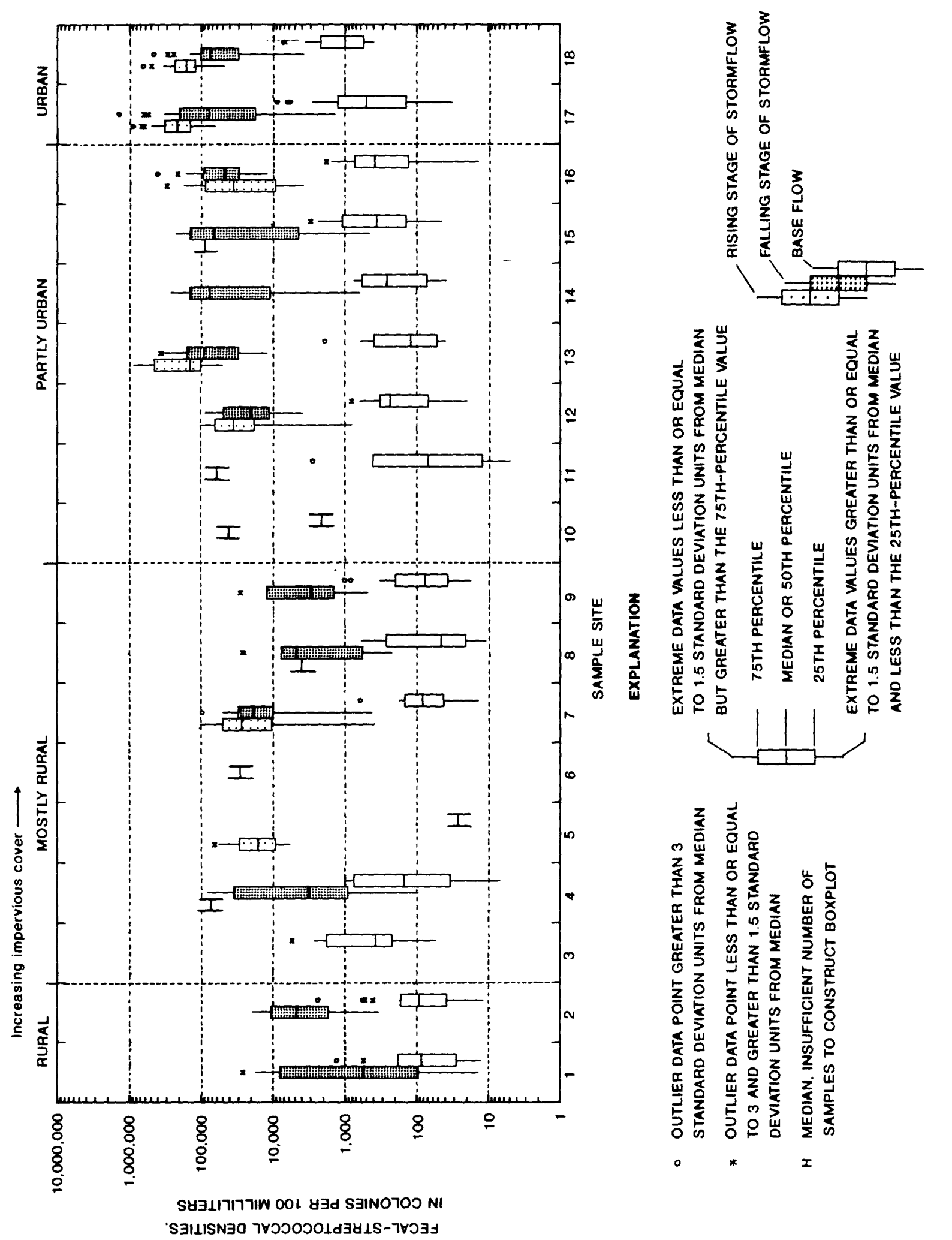

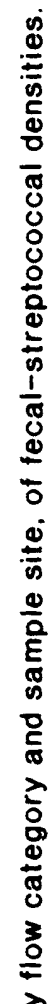

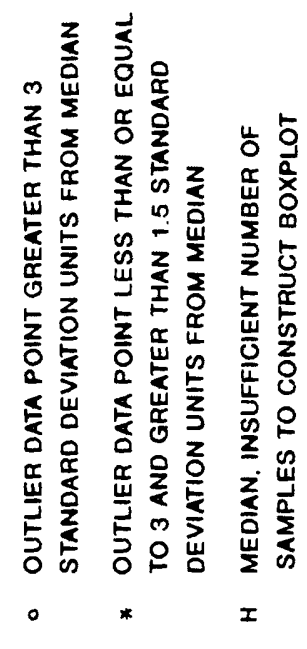

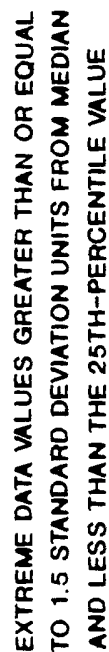

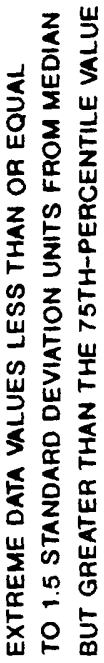


from one or more of the following: (1) their toxicity or cancer-causing effects on humans through consumption of the water or ingestion of aquatic fauna, (2) their effects on aquatic life, or (3) their persistence and bioconcentration in the environment.

Each of the 18 dissolved inorganic trace elements included in the analys is were detected at 1 or more of the 18 sample sites (table 5). Analyses were made for aluminum, boron, coba1t, lithium, nicke1, and strontium prior to 1977 only. These trace elements are discussed at the end of this section.

Detectable differences in the concentrations of the dissolved inorganic trace elements among the sample sites are shown in figures 13-20. The boxplots for most of these trace elements were constructed using a maximum likelihood statistical procedure to calculate the distribution of the traceelement concentrations. This procedure was necessary when the analyses were done using several detection thresholds during the sample collection (Helsel and Cohn, 1988). Only the smallest detection threshold is shown in figures 13-20 for trace elements with more than one detection threshold. Although most of the water sampled for this study is not directly used as a public water supply, the U.S. Environmental Protection Agency (1986a,b) National Primary and Secondary Drinking-Water Regulations are useful for comparison purposes and for some understanding of the potential problems associated with the presence of each inorganic trace element.

Smal1 concentrations of dissolved arsenic were detected in 73 percent of the 397 samples analyzed (table 5). This trace element was detected at all 18 sample sites, but was present in concentrations greater than or equal to the Environmental Protection Agency (1986a) Maximum Contaminant Leve 1 (MCL) of 50 $\mu \mathrm{g} / \mathrm{L}$ (micrograms per $1 \mathrm{iter}$ ) in only one sample, which was collected at site 18 (fig. 13). The larger concentrations of arsenic were detected at the sample sites with urban drainage basins and at some sites with partly urban basins, as illustrated in figure 13. Except for sample sites 16, 17, and 18, the median concentration of this trace element does not exceed the detection threshold of $1.0 \mu \mathrm{g} / \mathrm{L}$.

Dissolved barium was detected in 73 percent of the 346 samples analyzed (table 5). Larger and more variable concentrations of barium are evident in samples from sites with urban and partly urban drainage basins (fig. 14). Maximum likelihood was used to compute the distribution of dissolved barium because two detection thresholds were used during the sampling period. Water samples from site 3 probably are influenced by the unpermitted effluent discharge listed in table 2 for this drainage basin. Water from site 16 is known to contain some treated effluent. However, all samples collected at site 16 had barium concentrations much less than the U.S. Environmental Protection Agency (1986a) MCL of $1,000 \mu \mathrm{g} / \mathrm{L}$.

Concentrations of dissolved cadmium greater than the detection threshold were present in only 5 percent of the 402 samples analyzed (table 5). This element was detected at concentrations greater than or equal to the U.S. Environmental Protection Agency (1986a) $\mathrm{MCL}$ of $10 \mu \mathrm{g} / \mathrm{L}$ in five samples, although no relation was discernible between the concentrations and the development classification of the corresponding drainage basin.

Dissolved chromium was detected in only 8 percent of the 405 water samples analyzed (table 5). Dissolved chromium is considered less toxic than 


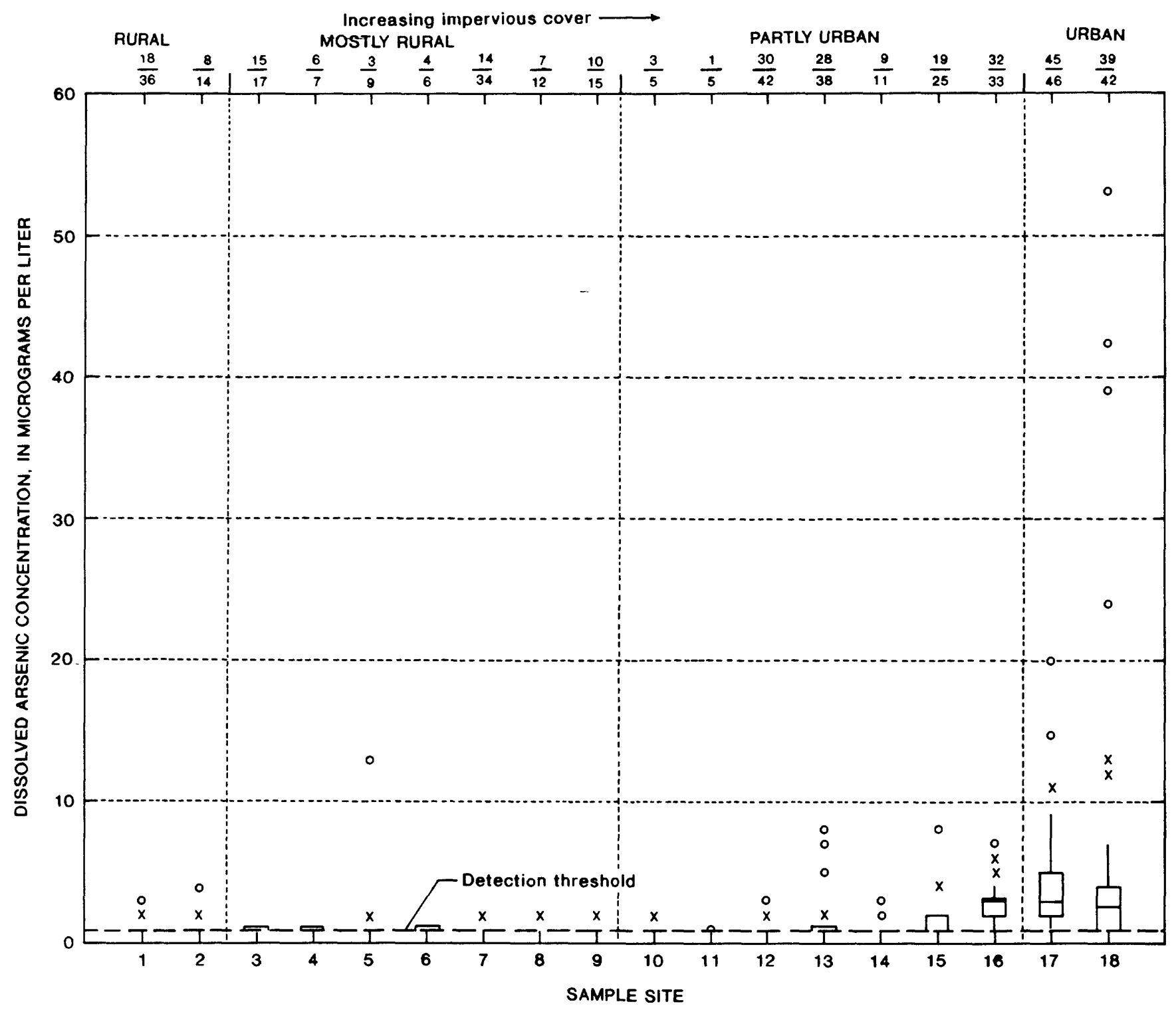

EXPLANATION

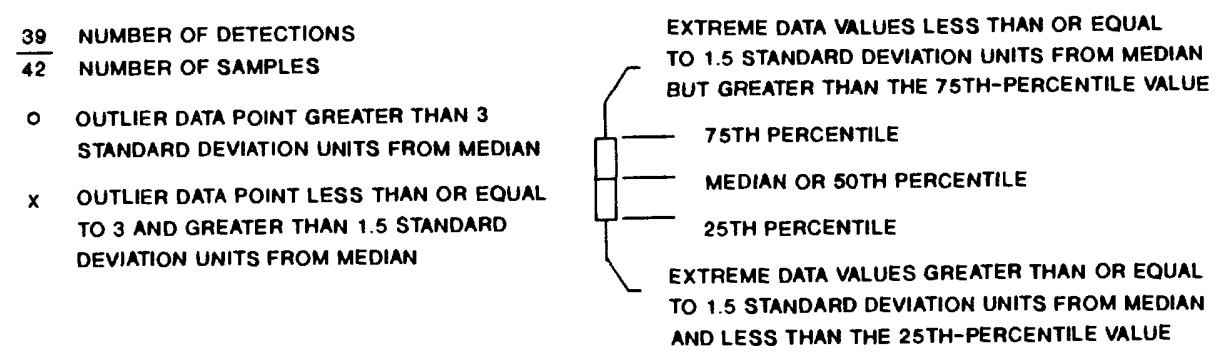

Figure 13.--Boxplots showing the distribution, by sample site, of dissolved arsenic concentrations. 


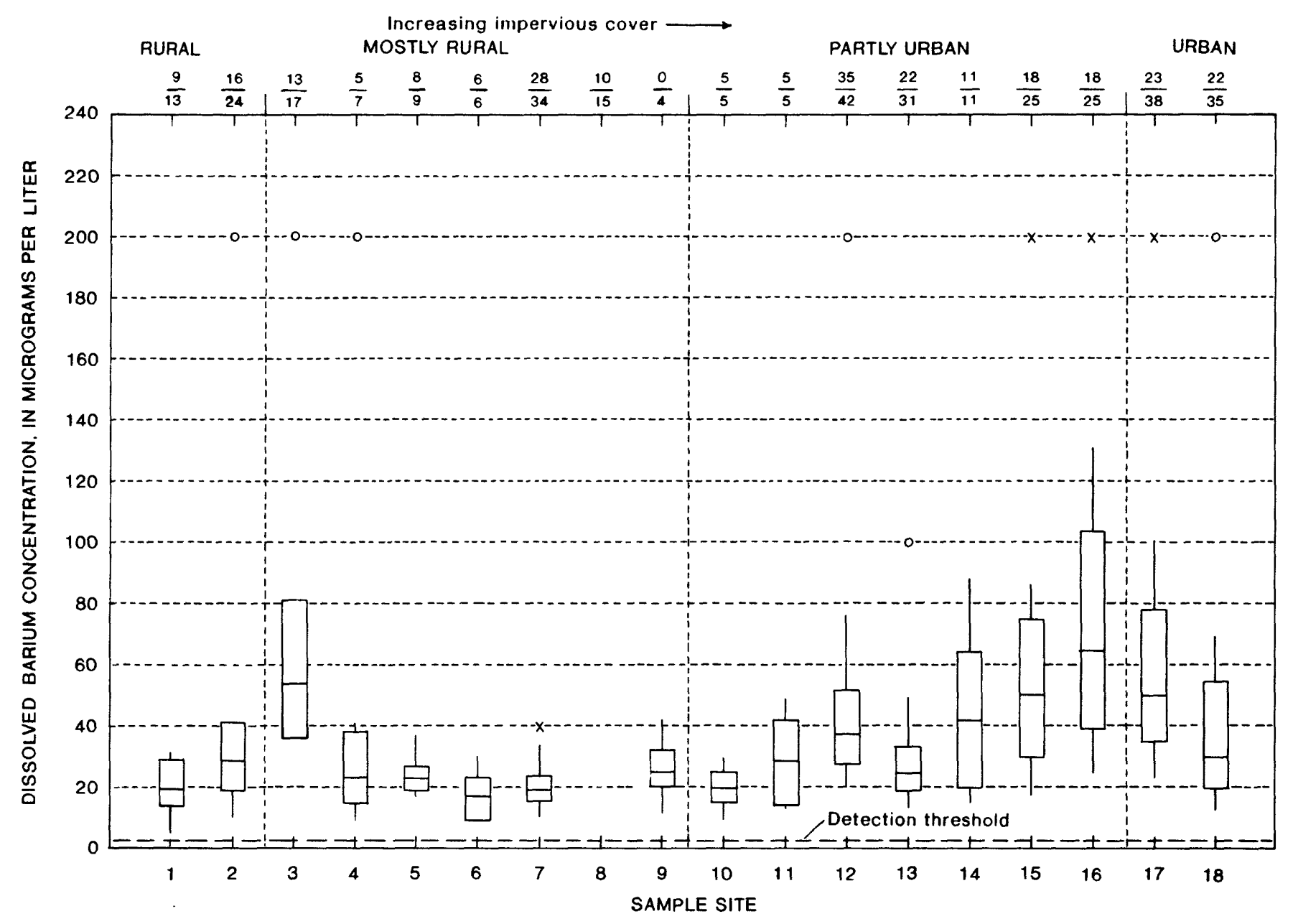

EXPLANATION

9 NUMBER OF DETECTIONS NUMBER OF SAMPLES

- OUTLIER DATA POINT GREATER than 3 STANDARD DEVIATION UNITS FROM MEDIAN

$x$ OUTLIER DATA POINT LESS THAN OR EQUAL TO 3 AND GREATER THAN 15 STANDARD DEVIATION UNITS FROM MEDIAN

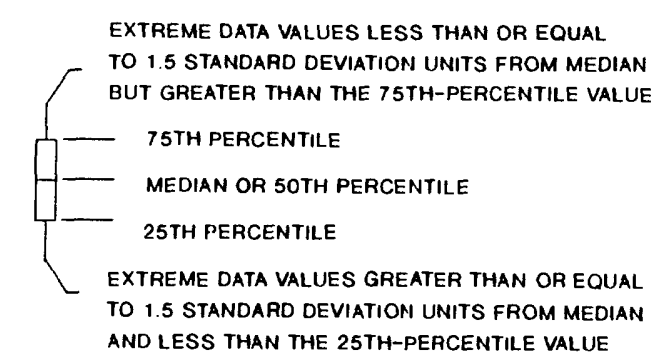

EXTREME DATA VALUES LESS THAN OR EQUAL

TO 1.5 STANDARD DEVIATION UNITS FROM MEDIAN

BUT GREATER THAN THE 75TH-PERCENTILE VALUE

25TH PERCENTILE AND LESS THAN THE 25TH-PERCENTILE VALUE

Figure 14.--Boxplots showing the distribution, by sample site, of dissolved barium concentrations. 
Table

$[\mu \mathrm{g} / \mathrm{L}$, micrograms

5.--Summary of inorganic trace elements

(dissolved) threshold

( $\mu \mathrm{g} / \mathrm{L})$

Arsenic

Aluminum ${ }^{2}$

Barium

10,100

Boron'

2,100

20

Cadmium

$1,2,3$

Chromium

Cobalt 2

$1,2,10,20$

Copper

2

Iron

Lead

Lithium²

$, 2,10$

$3,9,10$

Manganese

Mercury

5,10

Nickel ${ }^{2}$

10

Selenium

Silver

Stront ium ${ }^{2}$

Zinc

$$
1,2,10
$$

$.1, .5$

$23 / 41$

1,2

10

$$
3,12,20
$$

$161 / 399$

with detectable

concentrations

per number

of samples

291/397

$30 / 51$

$254 / 346$

$43 / 48$

$19 / 402$

$33 / 405$

$2 / 50$

222/399

266/399

48/399

$$
8 / 41
$$

$215 / 398$

21/397

41/359

41/41

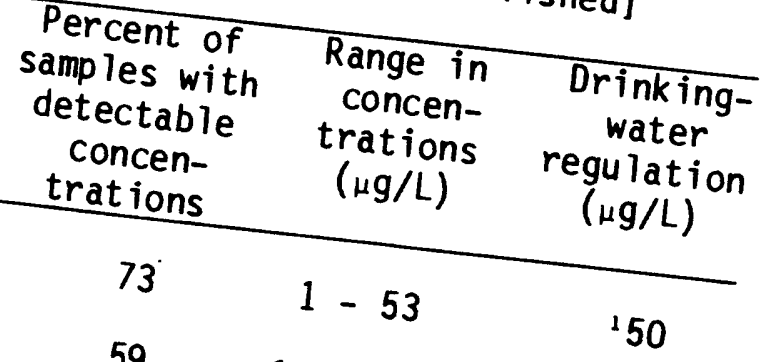

59

$10-70$

73

90

$5-200$

$30-220$

5

8

$1-42$

$2-60$

4

2

56

67

12

20

54

5

56

11

$12 / 359$

3

100

$1-23$

$3-460$

$5-25$

$20-30$

1 - 540

$.1-4.4$

$2-8$

$1-3$

$1-2$

${ }^{1} 10$

150

40

11,000

watation
$\mathrm{gg} / \mathrm{L})$

政

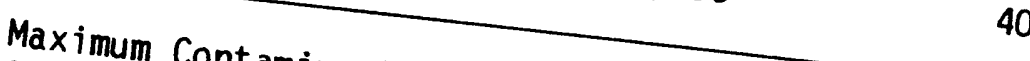

Analyzed at six sinant Leve

Secondary Maximum sites prior to 1977 . Envonmental

1986b).

only.

Protection Agency, 1986a).

$3-220$ 
several of the other heavy metals; only at site 2 did the concentration of chromium exceed the U.S. Environmental Protection Agency (1986a) MCL of 50 $\mu \mathrm{g} / \mathrm{L}$. A relation was not apparent between the concentrations of chromium in the samples analyzed and the development classification of the corresponding drainage basin.

Dissolved copper was detected in 56 percent of the 399 water samples analyzed (table 5). Copper is toxic to fish at concentrations less than the U.S. Environmental Protection Agency (1986b) Secondary Maximum Contaminant Level (SMCL) of $1,000 \mu \mathrm{g} / \mathrm{L}$, which is a taste threshold for this constituent. The distribution of the concentrations in samples (fig. 15) tended to be larger at the two sites (17 and 18) with urban drainage basins, although the concentrations of dissolved copper from samples collected at all sites was minimal compared to the SMCL. Maximum likelihood was used to compute the distribution of dissolved copper because three detection thresholds were used during the sampling period.

The concentration of dissolved iron was greater than the detection threshold in 67 percent of the 399 water samples (table 5). The U.S. Environmental Protection Agency (1986b) SMCL of $300 \mu \mathrm{g} / \mathrm{L}$ for iron a 1so is based on taste threshold. The iron concentrations in only three of all the samples collected were greater than the suggested SMCL. The number of detections and the distribution of concentrations were greater at the sites with urban and partly urban drainage basins although the concentrations of iron were not large (fig. 16). The large concentrations of iron in samples from site 6 could not be explained. Maximum likelihood was used to compute the distribution of dissolved iron because three different detection thresholds were used during the sampling period.

Dissolved lead was detected in 12 percent of the 399 samples analyzed (table 5). Lead was not detected in any samples in concentrations greater than the U.S. Environmental Protection Agency (1986a) MCL of $50 \mu \mathrm{g} / \mathrm{L}$. The smal1 number of samples with concentrations above the detection threshold did not appear to be related to the development classification of the drainage basins.

Concentrations of dissolved manganese were greater than laboratory detection thresholds in 54 percent of the 398 water samples (table 5). The U.S. Environmental Protection Agency (1986b) SMCL of $50 \mu \mathrm{g} / \mathrm{L}$ of manganese for public water supplies results from a tendency of water with large concentrations of dissolved manganese to deposit black oxide stains. Six sites had samples with dissolved manganese concentrations greater than the SMCL. On1y site 16 on Williamson Creek and site 6 on Little Bear Creek had median concentrations substantially larger than those from the other sites (fig. 17), which could possibly be the result of effluent discharges to this drainage and resultant oxidizing conditions. Maximum likelihood was used to compute the distribution of dissolved manganese because three detection thresholds were used during the sampling period.

Dissolved mercury was detected in only 5 percent of the 397 samples analyzed (table 5). Mercury had the smallest U.S. Environmental Protection Agency (1986a) MCL for drinking water of all the elements. Of the samples collected, only one sample at site 7 and one sample at site 13 had concentrations exceeding the $2.0 \mu \mathrm{g} / \mathrm{L}$ limit. The distribution of concentrations greater than the detection threshold does not appear to be related to the development classification of the drainage basins. 


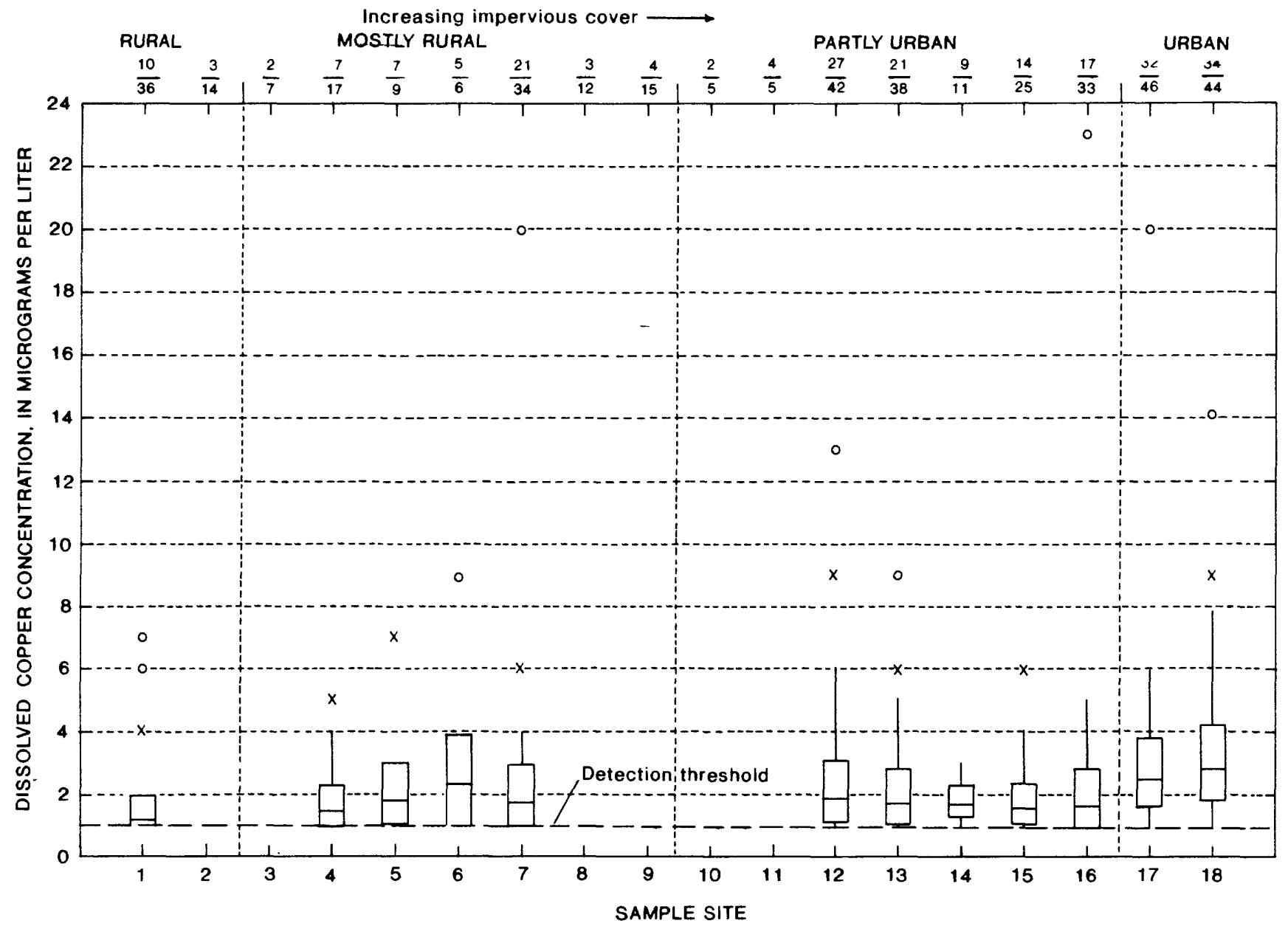

EXPLANATION

$\frac{10}{36}$ NUMBER OF DETECTIONS

- OUTLIER DATA POINT GREATER THAN 3 STANDARD DEVIATION UNITS FROM MEDIAN

$x$ OUTLIER DATA POINT LESS THAN OR EQUAL TO 3 AND GREATER THAN 1.5 STANDARD DEVIATION UNITS FROM MEDIAN
EXTREME DATA VALUES LESS THAN OR EQUAL

TO 15 STANDARD DEVIATION UNITS FROM MEDIAN BUT GREATER THAN THE 75TH-PERCENTILE VALUE

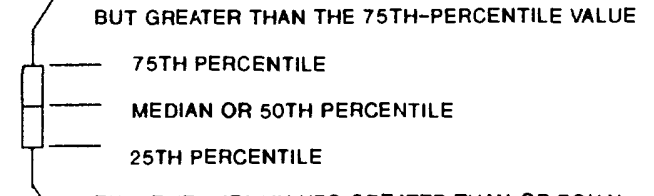

EXTREME DATA VALUES GREATER THAN OR EQUAL TO 15 STANDARD DEVIATION UNITS FROM MEDIAN AND LESS THAN THE 25TH-PERCENTILE VALUE

Figure 15.--Boxplots showing the distribution, by sample site, of dissolved copper concentrations. 


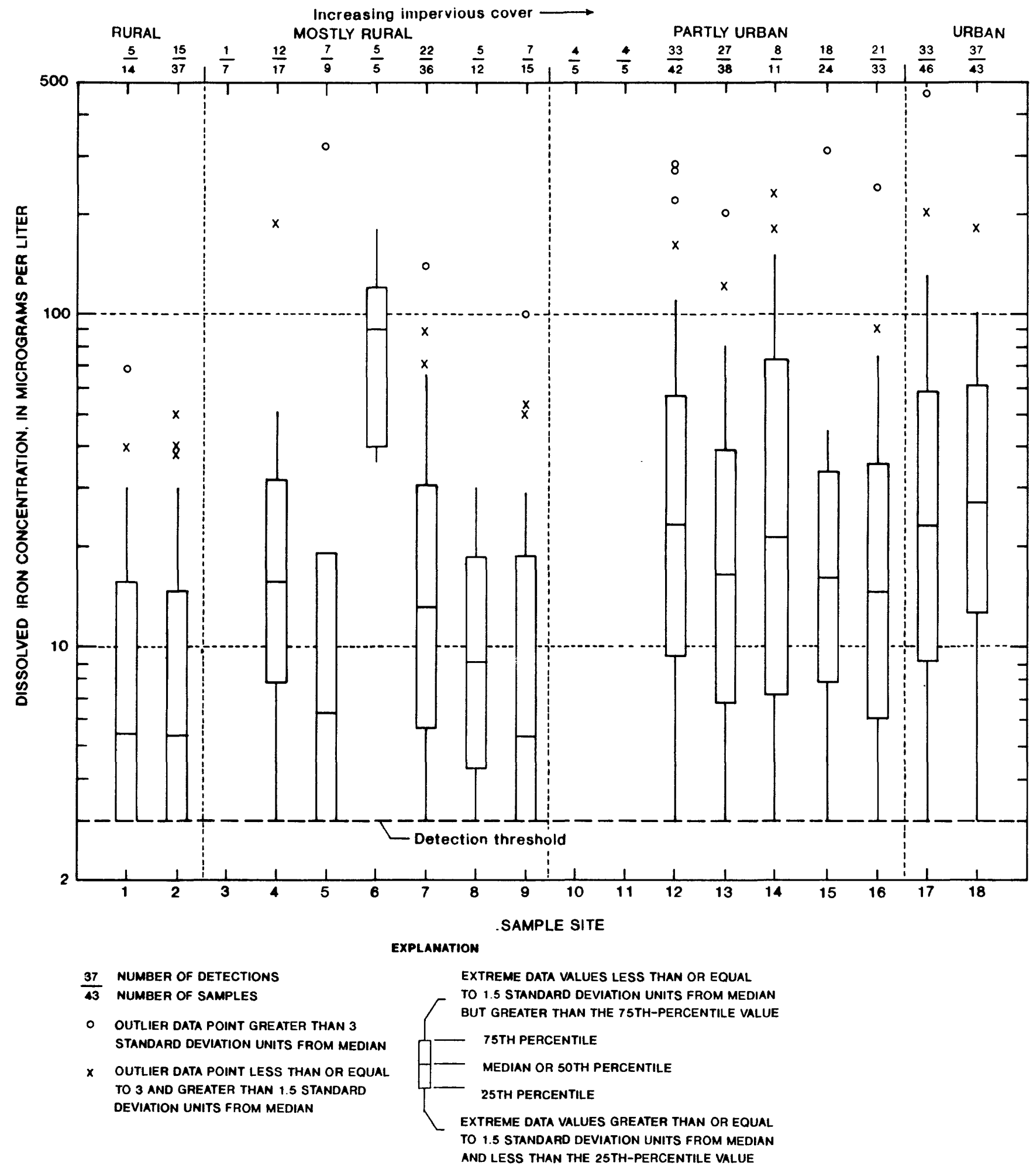

Figure 16.--Boxplots showing the distribution, by sample site, of dissolved iron concentrations. 


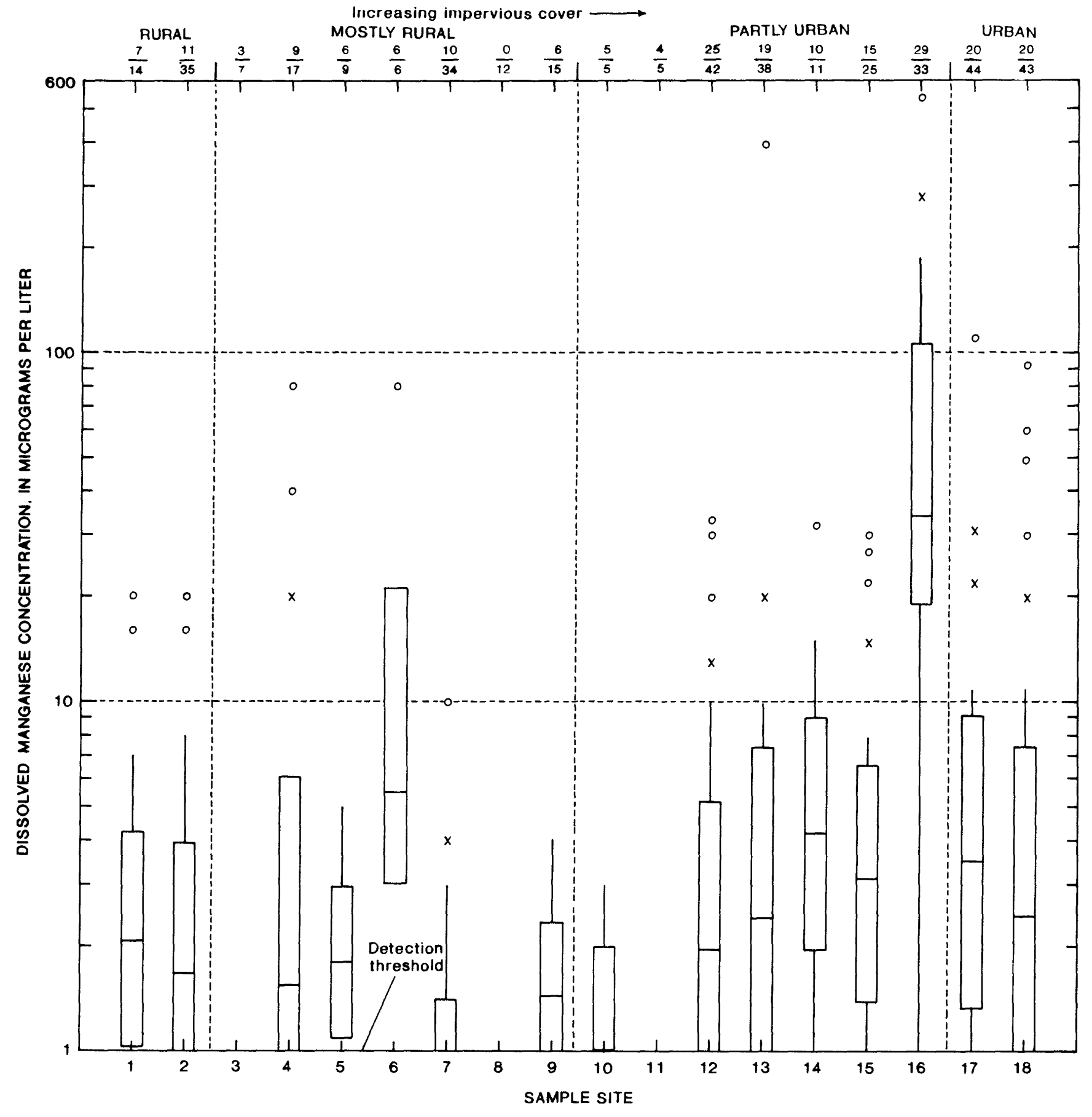

EXPLANATION
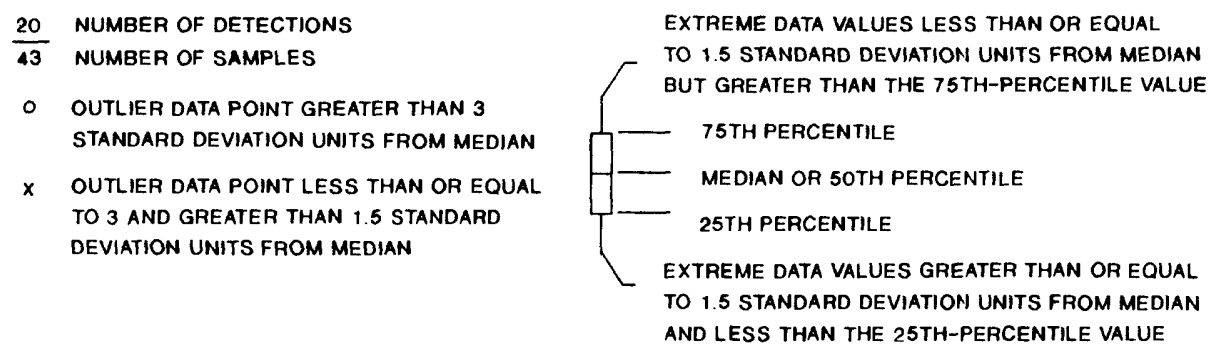

Figure 17.--Boxplots showing the distribution, by sample site, of dissolved manganese concentrations. 
Dissolved selentum and dissolved silver were detected in 11 and 3 percent, respectively, of the 359 samples analyzed (table 5). Although selenium is regarded as an essential nutritional element in small amounts, these trace elements have MCLs set by the U.S. Environmental Protection Agency (1986a) for public drinking-water supplies because of their respective toxicity to humans and aquatic life. The distributions of samples with concentrations greater than the detection threshold for either of these constituents were not related to the degree of urbanization.

Dissolved zinc was present in detectable concentrations in 40 percent of the 399 samples (table 5). Zinc is essential to animal and plant metabolism and the U.S. Environmental Protection Agency (1986b) SMCL is based on a taste threshold. Only two samples had concentrations of dissolved zinc greater than $100 \mu \mathrm{g} / \mathrm{L}$, and the concentrations were substantially below the SMCL of 5,000 $\mu \mathrm{g} / \mathrm{L}$ for drinking-water supplies. The number of detections and distributions of concentrations were greater at the sites with urban and partly urban drainage basins, even though the concentrations of zinc were not large (fig. 18). Maximum likelihood was used to compute the distribution of dissolved zinc because three detection thresholds were used during the sampling period.

Water samples were analyzed for six of the dissolved inorganic trace elements (a luminum, boron, cobalt, lithium, nickel, and strontium) prior to 1977 only. These six elements were found in 59, 90, 4, 20, 56, and 100 percent of the water samples, respectively (table 5), from only six of the sample sites $(2,8,13,16,17$, and 18). Drinking-water supply MCLs do not exist for these trace elements because they are not toxic in amounts normally present in water. Dissolved boron and strontium are the only two elements of these six that had any observable variation in concentration from site to site, as shown in figures 19 and 20. The concentration of dissolved boron was slightly larger at site 16, which includes an inflow of treated effluent, than at the other sites. Treated effluent usually contains relatively large concentrations of dissolved boron from detergents and other cleaning agents, along with minor contributions from human wastes and household and industrial chemicals. The presence of celestite crystals in the Glen Rose Formation probably is responsible for larger concentrations of dissolved strontium collected at site 8 (E.T. Baker, Jr., U.S. Geological Survey, oral commun., 1987) and plotted in figure 20.

\section{Synthet ic Organic Compounds}

Twenty-two of a group of 42 synthet ic organic compounds investigated were detected in samples collected for this study. The detection of a particular compound is primarily a function of the use of that compound in the basin draining to each site sampled, the solubility of the compound in water, the degree of adsorption on the suspended-solids part of an unfiltered sample, and the detection threshold of the particular chemical analysis used. The healthrelated concern about some of these synthetic organic compounds comes from their potential toxicity or cancer-causing effects on humans through consumption of the water or ingestion of aquatic fauna, their effects on aquatic life, or their related persistence and bioconcentration in the environment. The group of synthetic organic compounds for which chemical analyses were performed and the compounds that were detected at sites were grouped by the development classification of their contributing drainage basins (table 6). 


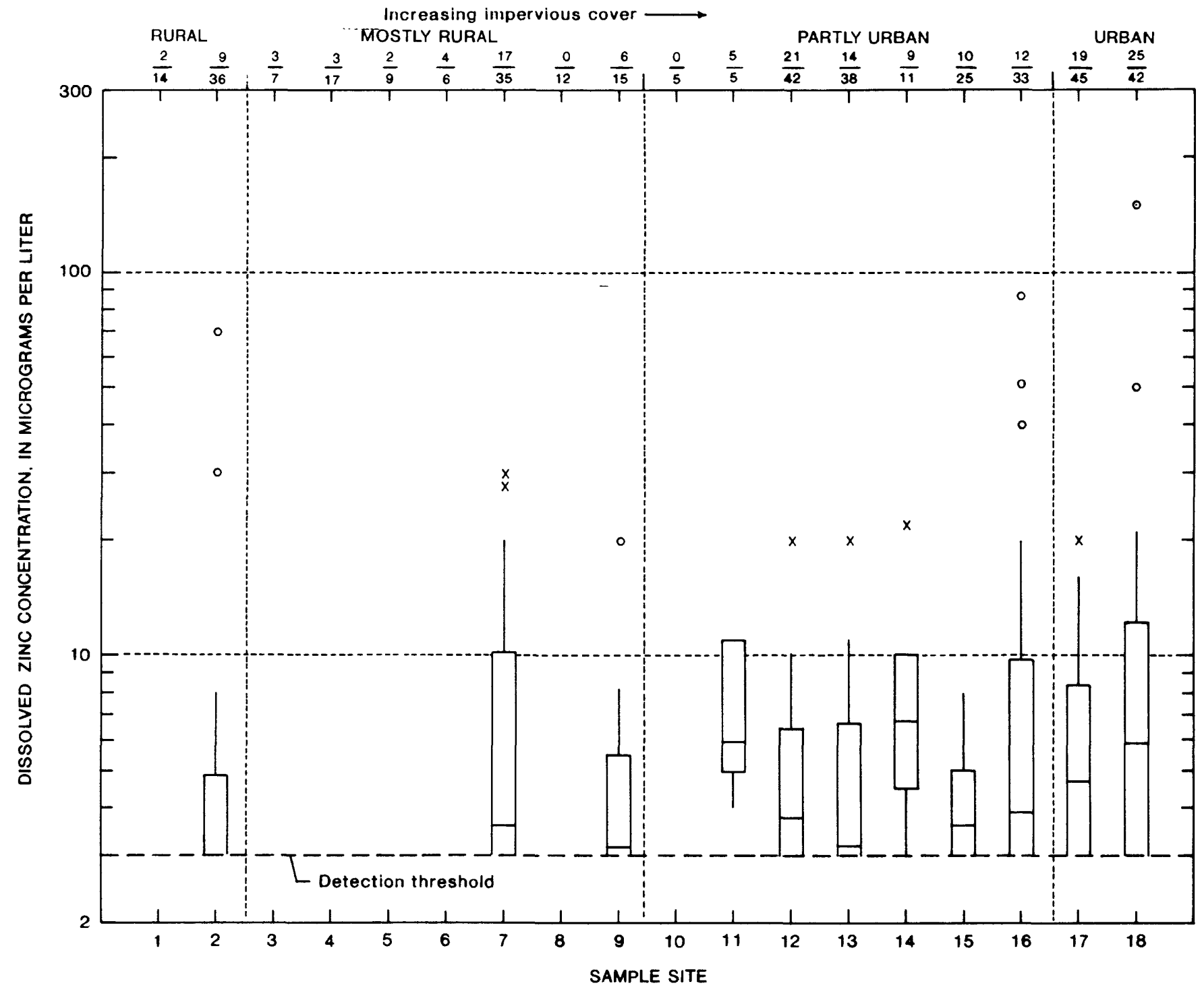

EXPLANATION
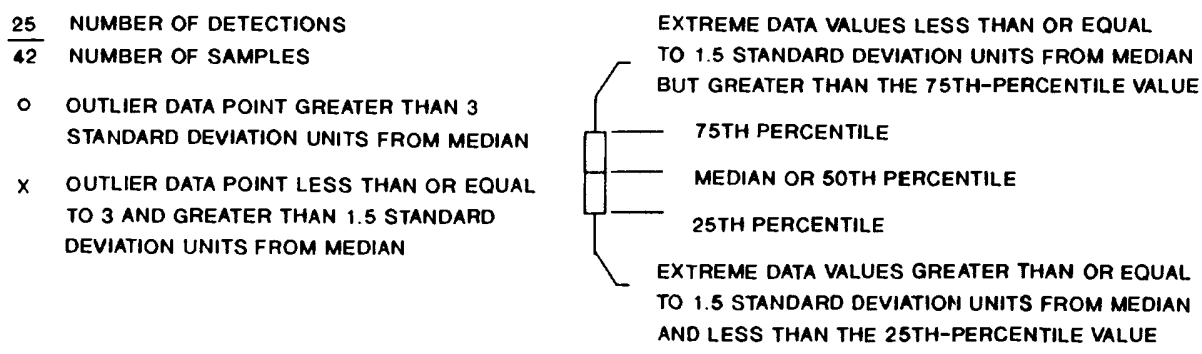

Figure 18.--Boxplots showing the distribution, by sample site, of dissolved zinc concentrations. 


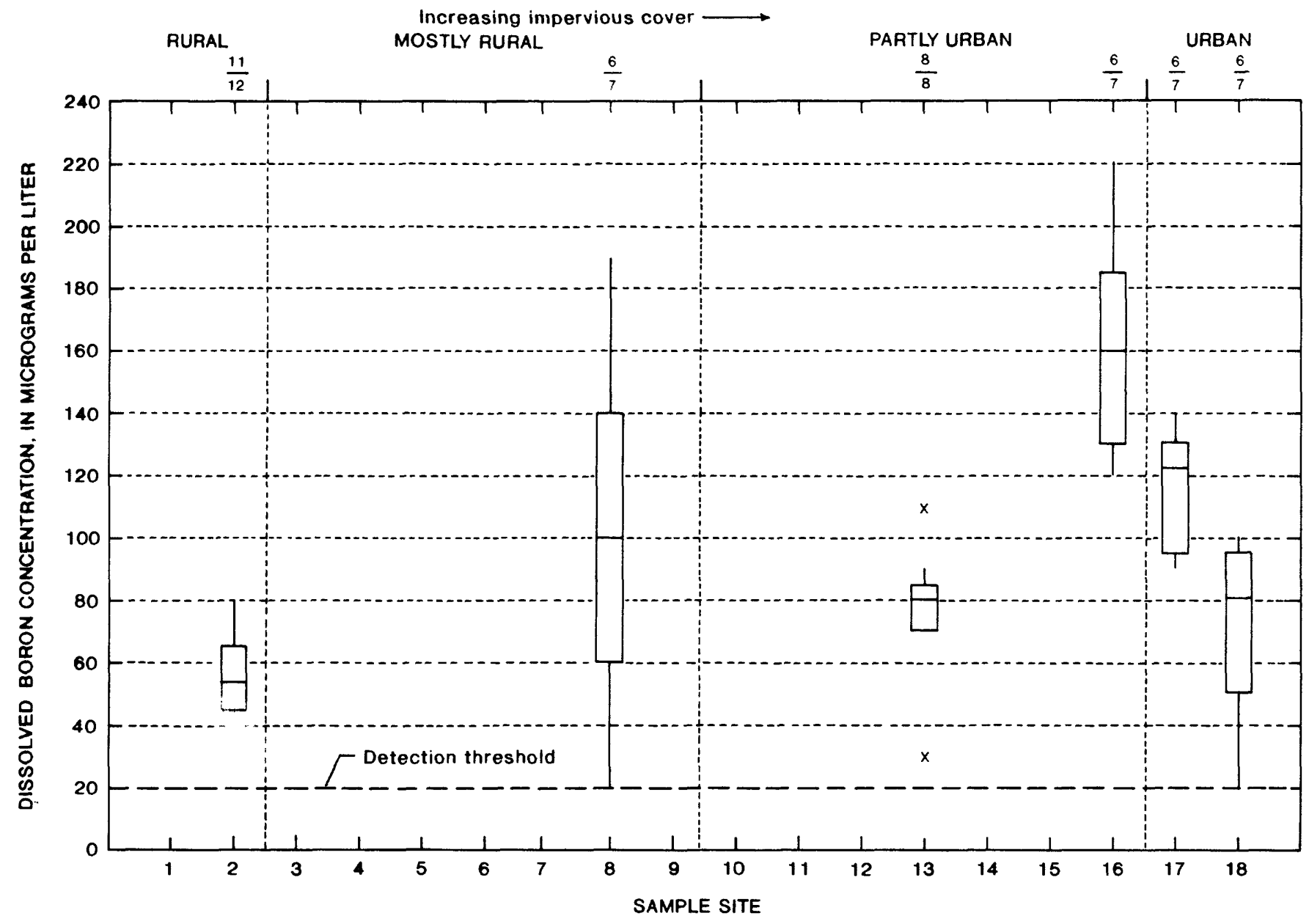

EXPLANATION

$\frac{6}{7}$ NUMBER OF DETECTIONS

$x$ OUTLIER DATA POINT LESS THAN OR EQUAL TO 3 AND GREATER THAN 1.5 STANDARD DEVIATION UNITS FROM MEDIAN
EXTREME DATA VALUES LESS THAN OR EQUAL

TO 1.5 STANDARD DEVIATION UNITS FROM MEDIAN

BUT GREATER THAN THE 75TH-PERCENTILE VALUE

75TH PERCENTILE

MEDIAN OR 5OTH PERCENTILE

25TH PERCENTILE

EXTREME DATA VALUES GREATER THAN OR EQUAL TO 1.5 STANDARD DEVIATION UNITS FROM MEDIAN AND LESS THAN THE 25TH-PERCENTILE VALUE

Figure 19.--Boxplots showing the distribution, by sample site, of dissolved boron concentrations. 


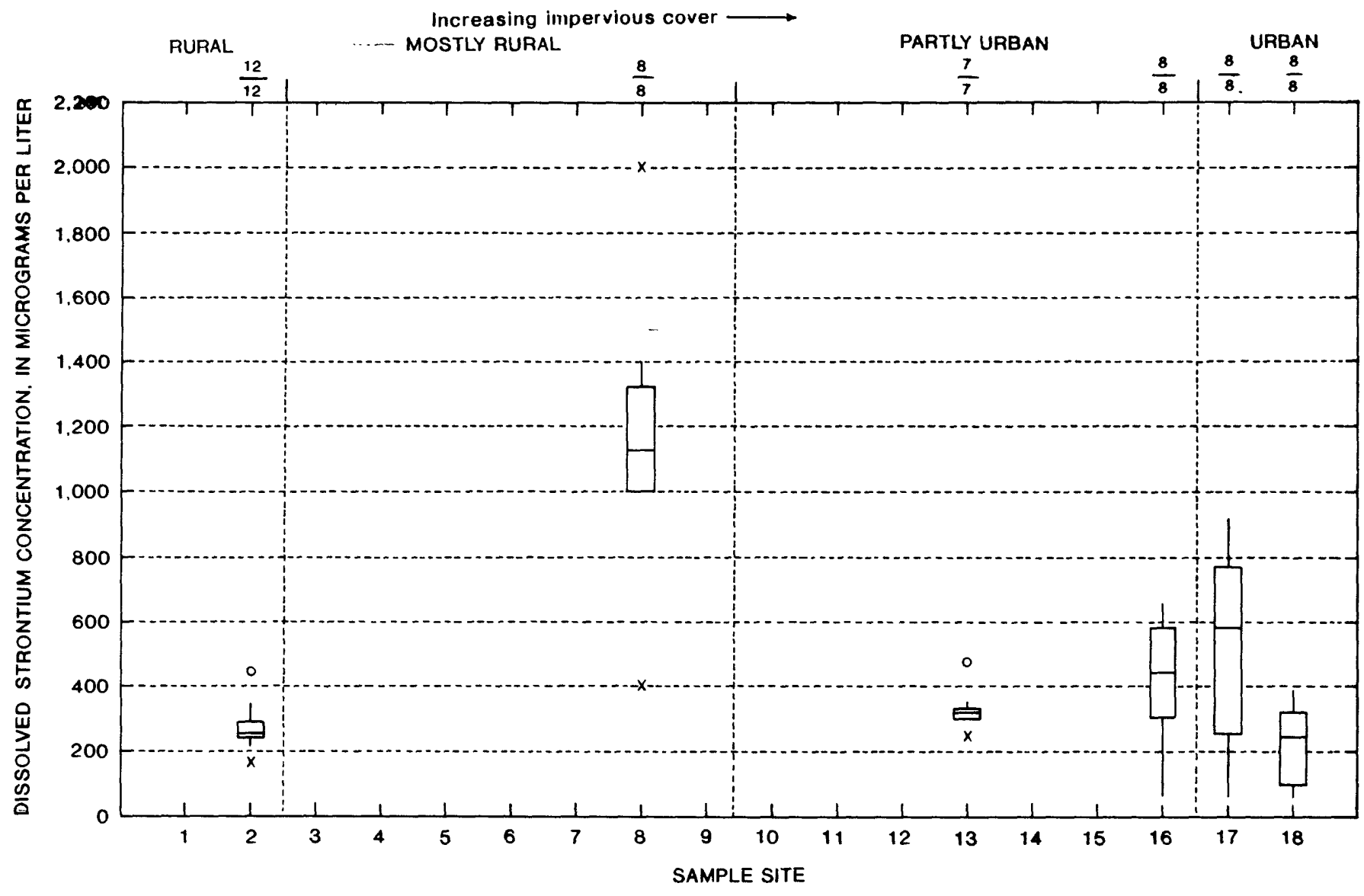

EXPLANATION

$\frac{8}{8}$ NUMBER OF DETECTIONS

NUMBER OF SAMPLES

- OUTLIER DATA POINT GREATER THAN 3 STANDARD DEVIATION UNITS FROM MEDIAN

$x$ OUTLIER DATA POINT LESS THAN OR EQUAL TO 3 AND GREATER THAN 1.5 STANDARD DEVIATION UNITS FROM MEDIAN
EXTREME DATA VALUES LESS THAN OR EQUAL

TO 1.5 STANDARD DEVIATION UNITS FROM MEDIAN BUT GREATER THAN THE 75TH-PERCENTILE VALUE

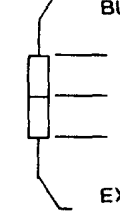
75TH PERCENTILE MEDIAN OR 5OTH PERCENTILE 25TH PERCENTILE

EXTREME DATA VALUES GREATER THAN OR EOUAL TO 1.5 STANDARD DEVIATION UNITS FROM MEDIAN AND LESS THAN THE 25TH-PERCENTILE VALUE

Figure 20.--Boxplots showing the distribution, by sample site, of dissolved strontium concentrations. 
Table 6.--Summary of analyzed synthet ic organic compounds

[ $\mathrm{H} / \mathrm{L}$, micrograms per liter; --, not detected]

\begin{tabular}{|c|c|c|c|c|c|c|c|}
\hline Compound & $\begin{array}{l}\text { Detection } \\
\text { threshold } \\
(\mu / L)\end{array}$ & $\begin{array}{c}\text { Number of samples } \\
\text { with detectable } \\
\text { concentrations } \\
\text { per number } \\
\text { of analyses } \\
\end{array}$ & $\begin{array}{l}\text { Range in } \\
\text { concen- } \\
\text { trations } \\
(\mu / L)\end{array}$ & $\frac{\text { deve }}{\text { Rurat }}$ & $\begin{array}{c}\text { etection } \\
\text { opment } c \\
\text { Mostiy } \\
\text { rural }\end{array}$ & $\begin{array}{l}\text { ratiob } \\
\text { Massific } \\
\text { Partiy } \\
\text { urban }\end{array}$ & $\begin{array}{l}y \\
\text { ation } \\
\text { Urban }\end{array}$ \\
\hline $\begin{array}{l}\text { Industrial compounds } \\
\text { Polychlorinated biphenyls } \\
\text { Polychlorinated naphthalenes }\end{array}$ & $\begin{array}{r}0.1 \\
.1\end{array}$ & $\begin{array}{l}3 / 179 \\
0 / 105\end{array}$ & $\begin{array}{c}0.1 \\
--\end{array}$ & $\begin{array}{c}0 / 20 \\
--\end{array}$ & $\begin{array}{c}0 / 49 \\
--\end{array}$ & $\begin{array}{c}0 / 64 \\
-\end{array}$ & $3 / 46$ \\
\hline $\begin{array}{l}\text { Insecticides } \\
\text { Organochlorine compounds } \\
\text { Aldrin } \\
\text { Chlordane } \\
\text { DDD } \\
\text { DDE } \\
\text { DDT }\end{array}$ & $\begin{array}{l}.01 \\
.1 \\
.01 \\
.01 \\
.01\end{array}$ & $\begin{array}{r}0 / 179 \\
20 / 179 \\
24 / 179 \\
26 / 179 \\
29 / 179\end{array}$ & $\begin{array}{l}.1-1.4 \\
.01-.38 \\
.01-.400 \\
.01-2.2\end{array}$ & $\begin{array}{l}-- \\
0 / 20 \\
0 / 20 \\
0 / 20 \\
0 / 20\end{array}$ & $\begin{array}{l}-\overline{0} \\
0 / 49 \\
0 / 49 \\
1 / 49 \\
1 / 49\end{array}$ & $\begin{array}{l}-- \\
3 / 64 \\
3 / 64 \\
3 / 64 \\
1 / 64\end{array}$ & $\begin{array}{l}17 / 46 \\
21 / 46 \\
22 / 46 \\
27 / 46\end{array}$ \\
\hline $\begin{array}{l}\text { Dieldrin } \\
\text { Endosulfan } \\
\text { Endrin } \\
\text { Heptochlor } \\
\text { Heptochlorepoxide }\end{array}$ & $\begin{array}{l}.01 \\
.01 \\
.01 \\
.01 \\
.01\end{array}$ & $\begin{array}{r}30 / 179 \\
0 / 144 \\
1 / 179 \\
5 / 179 \\
11 / 179\end{array}$ & $\begin{array}{l}.01-.12 \\
.04=- \\
.01=.03 \\
.01-.03\end{array}$ & $\begin{array}{l}0 / 20 \\
0 / 20 \\
0 / 20 \\
0 / 20\end{array}$ & $\begin{array}{l}0 / 49 \\
0 / 49 \\
0 / 49 \\
0 / 49\end{array}$ & $\begin{array}{l}1 / 64 \\
-- \\
0 / 64 \\
0 / 64 \\
1 / 64\end{array}$ & $\begin{array}{r}29 / 46 \\
-- \\
1 / 46 \\
5 / 46 \\
10 / 46\end{array}$ \\
\hline $\begin{array}{l}\text { Lindane } \\
\text { Methoxychlor } \\
\text { Mirex } \\
\text { Perthane } \\
\text { Toxaphene }\end{array}$ & $\begin{array}{l}.01 \\
.01 \\
.01 \\
.1 \\
1.0\end{array}$ & $\begin{array}{l}9 / 179 \\
0 / 43 \\
0 / 112 \\
1 / 105 \\
2 / 164\end{array}$ & $\begin{array}{c}.01-.03 \\
-- \\
2.0-- \\
1.0=--\end{array}$ & $\begin{array}{c}0 / 20 \\
-- \\
0 / 15 \\
0 / 19\end{array}$ & $\begin{array}{c}1 / 49 \\
-- \\
0 / 33 \\
0 / 36\end{array}$ & $\begin{array}{c}3 / 64 \\
-- \\
1 / 39 \\
0 / 64\end{array}$ & $\begin{array}{l}5 / 46 \\
-- \\
0-\overline{17} \\
2 / 46\end{array}$ \\
\hline $\begin{array}{l}\text { Organophosphorus compounds } \\
\text { Diazinon } \\
\text { Ethion } \\
\text { Malathion } \\
\text { Methyl parathion } \\
\text { Methyl trithion }\end{array}$ & $\begin{array}{l}.01 \\
.01 \\
.01 \\
.01 \\
.01\end{array}$ & $\begin{array}{r}94 / 179 \\
0 / 159 \\
20 / 177 \\
2 / 175 \\
0 / 159\end{array}$ & $\begin{array}{l}.01-.84 \\
.01-.14 \\
.01-.02 \\
--\end{array}$ & $\begin{array}{l}0 / 20 \\
0 / 20 \\
0 / 20 \\
--\end{array}$ & $\begin{array}{c}12 / 49 \\
0 / 48 \\
0 / 48 \\
--\end{array}$ & $\begin{array}{c}42 / 64 \\
6 / 64 \\
0 / 64 \\
--\end{array}$ & $\begin{array}{r}40 / 46 \\
14 / 45 \\
2 / 46 \\
--\end{array}$ \\
\hline $\begin{array}{l}\text { Parathion } \\
\text { Trithion }\end{array}$ & $\begin{array}{l}.01 \\
.01\end{array}$ & $\begin{array}{l}1 / 175 \\
0 / 159\end{array}$ & $=$ & $0 / 20$ & $0 / 48$ & $\begin{array}{c}0 / 64 \\
--\end{array}$ & $1 / 46$ \\
\hline $\begin{array}{l}\text { Carbonate compounds } \\
\text { Methomyl } \\
\text { Propham } \\
\text { Sevin }\end{array}$ & $\begin{array}{l}2.0 \\
2.0 \\
2.0\end{array}$ & $\begin{array}{l}0 / 85 \\
0 / 85 \\
0 / 85\end{array}$ & $\begin{array}{l}-- \\
-- \\
--\end{array}$ & $\begin{array}{l}-- \\
--\end{array}$ & $\begin{array}{l}-- \\
-- \\
--\end{array}$ & $\begin{array}{l}-- \\
--\end{array}$ & $\begin{array}{l}-- \\
-- \\
--\end{array}$ \\
\hline $\begin{array}{l}\text { Herbicides } \\
\text { Chlorophenoxy acid compounds } \\
2,4-D \\
2,4-D \mathrm{P} \\
2,4,5-\mathrm{T} \\
\text { Silvex }\end{array}$ & $\begin{array}{l}.01 \\
.01 \\
.01 \\
.01\end{array}$ & $\begin{array}{c}57 / 177 \\
0 / 21 \\
54 / 177 \\
7 / 176\end{array}$ & $\begin{array}{l}.01-.48 \\
--.01-1.0 \\
.01-2.9\end{array}$ & $\begin{array}{l}2 / 19 \\
\overline{1 / 19} \\
0 / 19\end{array}$ & $\begin{array}{l}7 / 51 \\
4 / 51 \\
1 / 50\end{array}$ & $\begin{array}{r}27 / 64 \\
-- \\
23 / 64 \\
1 / 64\end{array}$ & $\begin{array}{r}21 / 43 \\
24 / 43 \\
5 / 43\end{array}$ \\
\hline $\begin{array}{l}\text { Triazene compounds } \\
\text { Ametryne } \\
\text { Atratone } \\
\text { Atrazine } \\
\text { Cynazine } \\
\text { Cyprazine }\end{array}$ & $\begin{array}{l}.1 \\
.1 \\
.1 \\
.1 \\
.1\end{array}$ & $\begin{array}{r}0 / 94 \\
0 / 53 \\
15 / 94 \\
0 / 94 \\
0 / 53\end{array}$ & $\begin{array}{l}-- \\
.1- \\
-- \\
--\end{array}$ & $\begin{array}{c}-- \\
0 / 8 \\
-- \\
--\end{array}$ & $\begin{array}{c}-- \\
0 / 28 \\
-- \\
--\end{array}$ & $\begin{array}{c}-- \\
9 / 40 \\
-- \\
--\end{array}$ & $\begin{array}{l}-- \\
6 / 18 \\
-- \\
--\end{array}$ \\
\hline $\begin{array}{l}\text { Prometone } \\
\text { Prometryne } \\
\text { Propazine } \\
\text { Simazine } \\
\text { Simatone }\end{array}$ & $\begin{array}{l}.1 \\
.1 \\
.1 \\
.1 \\
.1\end{array}$ & $\begin{array}{r}41 / 94 \\
0 / 93 \\
0 / 93 \\
8 / 90 \\
0 / 53\end{array}$ & $\begin{array}{c}.1-8.4 \\
-- \\
-1-.8 \\
--\end{array}$ & $\begin{array}{c}0 / 8 \\
-- \\
0 / 8 \\
--\end{array}$ & $\begin{array}{c}2 / 28 \\
-- \\
0 / 24 \\
--\end{array}$ & $\begin{array}{c}21 / 40 \\
-- \\
3 / 40 \\
--\end{array}$ & $\begin{array}{c}18 / 18 \\
\overline{--} \\
5 / 18 \\
--\end{array}$ \\
\hline Simetryne & .1 & $0 / 94$ & -- & -- & -- & -- & -- \\
\hline
\end{tabular}


Of the 179 samples analyzed, 3 samples collected from sites with urban drainage basins (sites 17 and 18) had concentrations of a polychlorinated biphenyl compound that were equal to the detection threshold for that compound. Polychlorinated naphthalenes were not detected in any of the 105 samples analyzed.

\section{Insecticides}

Samples were analyzed for 15 organochlorine compounds, of which 11 were detected (table 6). Most of the organochlorine compounds probably are adsorbed on the suspended-solids part of the samples because they are slightly soluble in water. Several of these compounds either are banned or are highly restricted for private use; most have associated health advisories because of their persistence in the environment and their toxic or carcinogenic effects on man or aquatic life. A review of the number of detections for the sample sites, grouped by the development classification of the drainage basins as shown in table 6, indicates that most samples with concentrations of organochlorine compounds greater than detection thresholds are from sites with partly urban and urban basins.

Four of the seven organophosphorus compounds investigated were detected in the samples collected. Organophosphorus compounds are less persistent in the environment, are more soluble than are the organochlorine compounds, and have no health advisories associated with them. Diazinon and malathion were detected in 53 and 11 percent of all the samples analyzed, even though malathion is known to break down quickly in the alkaline soil types typical of the Aust in area (H.B. Mendieta, U.S. Geological Survey, retired, oral commun., 1986). The largest percentage of samples that had detectable concentrations of diazinon and malathion were at the sites with urban and partly urban drainage basins (table 6), even though diazinon was detected at all but 3 (sites 1, 2, and 3) of the 16 sites where samples were analyzed. The distribution of concentrations of diazinon for those sites with a sufficient number of samples with detectable concentrations to construct boxplots are shown in figure 21. Methy 1 parathion was detected twice at site 17 and parathion was detected once at site 18.

Carbonate compounds were not found in any of the samples, probably because of the large detection threshold and their short-term persistence in the environment.

\section{Herbicides}

Three of the four chlorophenoxy acid compounds for which samples were analyzed were detected. Chlorophenoxy acid compounds are less persistent in the environment than organochlorine-based insecticides and are highly soluble in aqueous solutions. The three herbicides detected in concentrations greater than the detection threshold have health advisories associated with them. Two of the detected herbicides, 2,4-D and 2,4,5-T, were detected in approximately 30 percent of all samples and were detected more commoniy in samples from sites with urban and partly urban drainage basins (table 6). Silvex was detected in only 4 percent of al1 samples collected and in about 12 percent of samples from sites with urban drainage basins. 


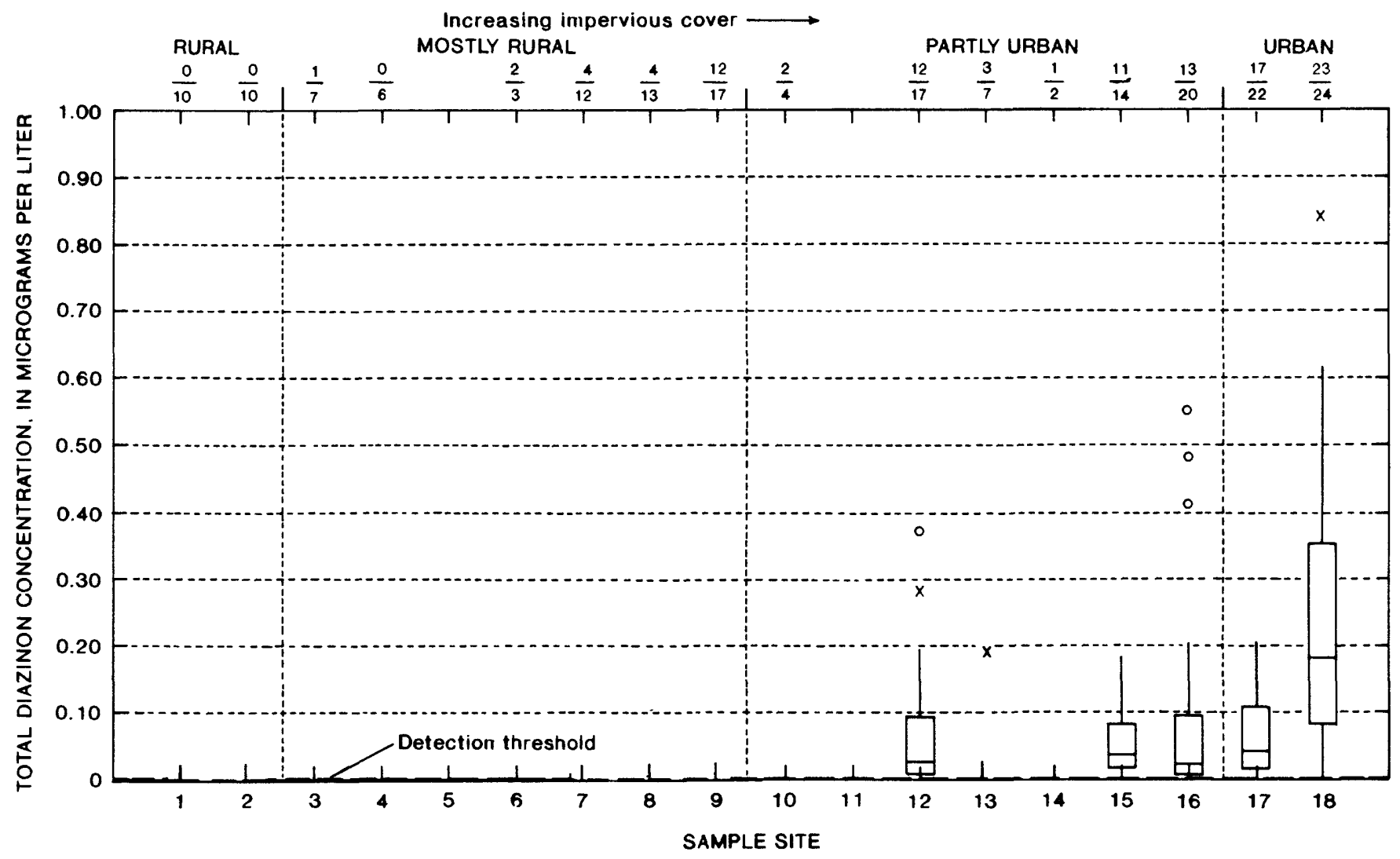

EXPLANATION

23 NUMBER OF DETECTIONS

NUMBER OF SAMPLES

- OUTLIER DATA POINT GREATER THAN 3 STANDARD DEVIATION UNITS FROM MEDIAN

$x$ OUTLIER DATA POINT LESS THAN OR EQUAL TO 3 AND GREATER THAN 1.5 STANDARD DEVIATION UNITS FROM MEDIAN

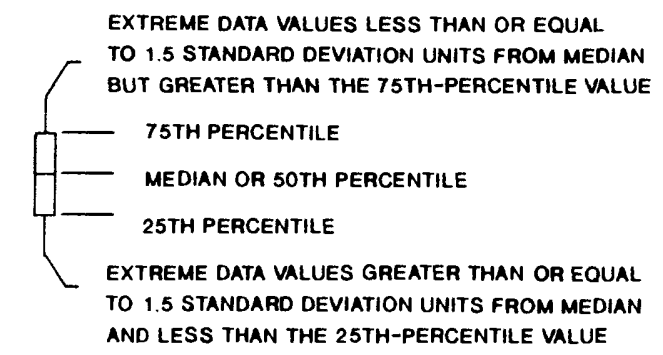

Figure 21.--Boxplots showing the distribution, by sample site, of total diazinon concentrations. 
Three of the 11 triazene compounds for which samples were analyzed were detected. Triazene compounds are not persistent in the environment, are highly soluble in aqueous solutions, and have no associated health advisories. Atrazine, prometone, and simazine were detected in 16, 44, and 9 percent of al1 samples analyzed, respectively, most often in samples collected at sites with urban and partly urban drainage basins (table 6).

\section{Radiochemical Constituents}

One or two water samples collected at each of 14 sites during the 1980 water year were analyzed for radiochemical constituents. The results of the radiochemical analyses are presented in table 7. The U.S. Environmental Protection Agency (1986a) MCLs in drinking water for radiochemical analysis are $5 \mathrm{pCi} / \mathrm{L}$ (picocuries per 1iter) for radium-226, $15 \mathrm{pCi} / \mathrm{L}$ for gross alpha activity, and $8 \mathrm{pCi} / \mathrm{L}$ for gross beta activity. The second sample taken during the rising stage of stormflow (April 25) at site 12 had suspended gross alpha and beta activity at a level greater than the $\mathrm{MCL}$. The concentration of dissolved uranium in a sample collected at site 10 is substantially greater than the concentrations for this element at the other sites. A drinking-water $M C L$ has not been established for uranium.

\section{RELATION BETWEEN URBANIZATION AND WATER QUALITY}

Differences in water quality among sample sites, development classifications, and flow categories generally may be assessed by inspection of the graphical and tabular data. Statistical comparisons were made, however, to substantiate differences in concentrations and densities of waterquality properties and constituents in samples collected during different flow categories (1) at each site, and (2) grouped by development classification.

\section{Comparisons Among Five Sites with Automatic Samplers}

Valid statistical tests could not be made for all 18 sites because of the differences in the number and distribution of samples at each site for each flow category. However, five of the six sites with automatic samplers (sites 7. 12, 13, 17, and 18) had a sufficient number of samples collected in each flow category to statistically compare the distribution of water-quality concentrations and densities.

For each water-quality constituent at each of the five sites, the baseflow median concentrations were smaller statistically than the median values from either the rising stage or falling stage of stormflow. For each site, the concentrations in samples collected during the rising stage were more variable than the concentrations in samples collected during the falling stage, which, in turn, were more variable than in those collected during base flow.

The differences between median concentrations of samples collected in the rising stage and falling stage varied with the amount of development upstream from each site. For each of the eight constituents at the two basins with urban classifications (sites 17 and 18), the rising-stage median concentrations were significantly larger than the corresponding falling-stage median concentrations. For site 13 (partly urban), the rising-stage median concentra- 


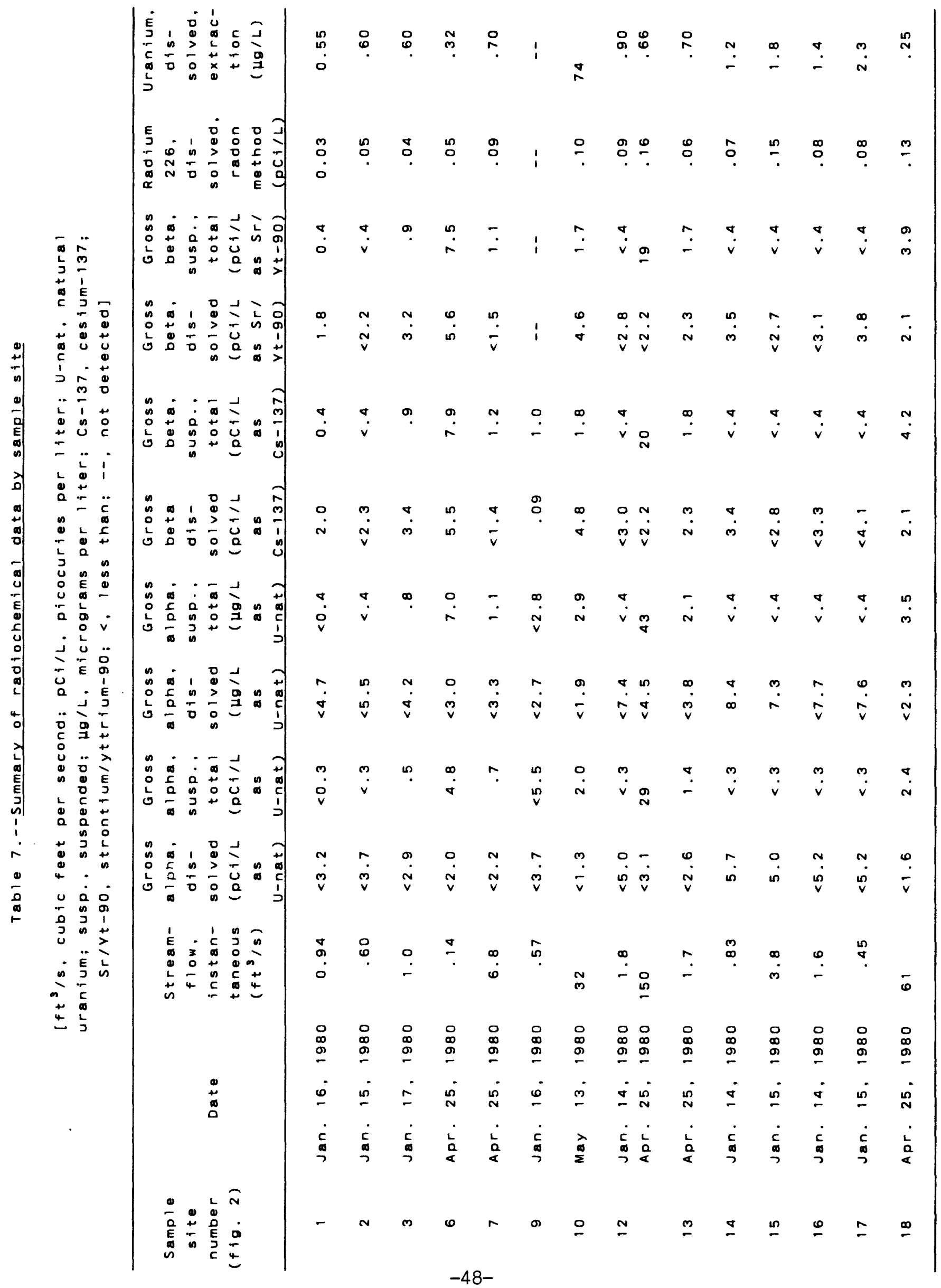


tion for each constituent was significantly larger than that of the falling stage, except for bacterial densities, which were not different at $\alpha<0.05$. For site 12 (partly urban), only the median suspended-solids concentrations in the rising stage and falling stage of stormflow were significantly different. For site 7 (mostly rural), the median concentrations for samples collected during the rising stage and falling stage were not significantly different. Furthermore, median concentrations of two constituents (BOD and total nitrogen) were larger in samples collected at site 7 during the falling stage of stormflow than during the rising stage. This is possible because the impervious surfaces in the drainage basin of this site are farther from the stream. The water-quality constituents would not reach the sample site until after streamflow had began to recede.

Some general conclusions may be inferred from the differences and similarities between concentrations in samples collected during the rising stage and falling stage. The concentrations in samples collected during the rising stage were more variable than those collected during the falling stage. The median concentrations in the rising stage and falling stage increased with increasing impervious cover upstream from the sites, and medians in the rising stage had larger increases than those in the falling stage. The significantly larger concentrations in samples collected during the rising stage were more apparent in the runoff from the more urban basins. The increased overland runoff and washoff from impervious surfaces is a possible explanation. The concentrations in samples collected during the rising stage would be expected to be more variable than those in the falling stage because of the nonhomogeneous land use that is characteristic of more urban sites. Runoff originating from different types of land use at varying distances from the sample site would account for this variability, whereas runoff from rural sites would be expected to be more homogeneous throughout the drainage basin. Samples collected during the falling stage could be expected to contain concentrations that are more uniformly distributed.

\section{Comparisons Among Four Development Classifications}

The 18 sample locations were grouped by four development classifications based on the percent of impervious cover, as described previously. This was done to facilitate comparison between sites with and without urban development and with uneven distributions of samples. Median concentrations of selected water-quality constituents for each of the four development classifications by flow category are given in table 8. Boxplots illustrating the distributions of concentrations and the discharge in samples collected from the three flow categories by development classification are presented in figures 22-29.

The median concentrations of dissolved solids were not related to development classifications in the rising stage and falling stage of stormflow (fig. 22). The base-flow median concentrations increased with increased development. The base-flow median concentrations were statistically different from each other at $\alpha<0.05$, except for the difference between concentrations for the urban and partly urban classifications.

The median concentrations of suspended solids in both stages of stormflow increased with increasing development; however, the rising-stage medians for the partly urban and urban classifications were significantly different at $\alpha$ $<0.10$, and the falling-stage medians for the urban and partly urban classifications were not significantly different (fig. 23, table 8). The base-flow 


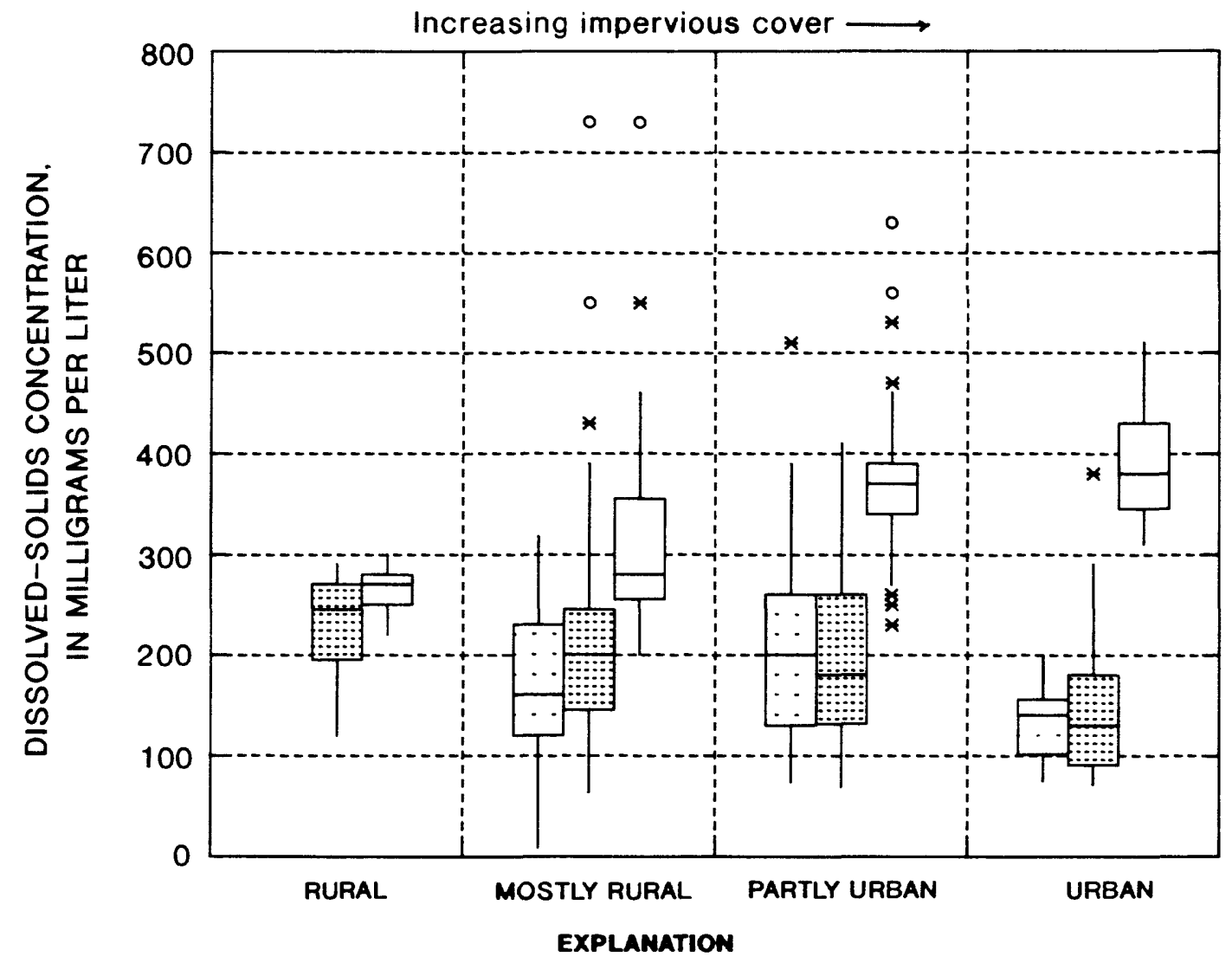

- OUTLIER DATA POINT GREATER THAN 3 STANDARD DEVIATION UNITS FROM MEDIAN

EXTREME DATA VALUES LESS THAN OR EQUAL TO 1.5 STANDARD DEVIATION UNITS FROM MEDIAN

* outlier data point less than or equal TO 3 AND GREATER THAN 1.5 STANDARD DEVIATION UNITS FROM MEDIAN

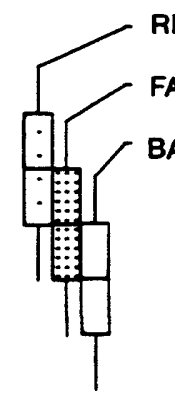

RISING STAGE OF STOFWFLOW

FALLING STAGE OF STORMFLOW

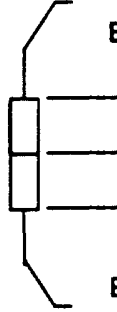
BUT GREATER THAN THE 75TH-PERCENTILE VALUE

\section{TH PERCENTILE}

MEDIAN OR 50TH PERCENTILE

25TH PERCENTILE

EXTREME DATA VALUES GREATER THAN OR EQUAL TO 1.5 STANDARD DEVIATION UNITS FROM MEDIAN AND LESS THAN THE 25TH-PERCENTILE VALUE

Figure 22.--Boxplots showing the distribution, by flow category and development classification, of dissolved-solids concentrations. 


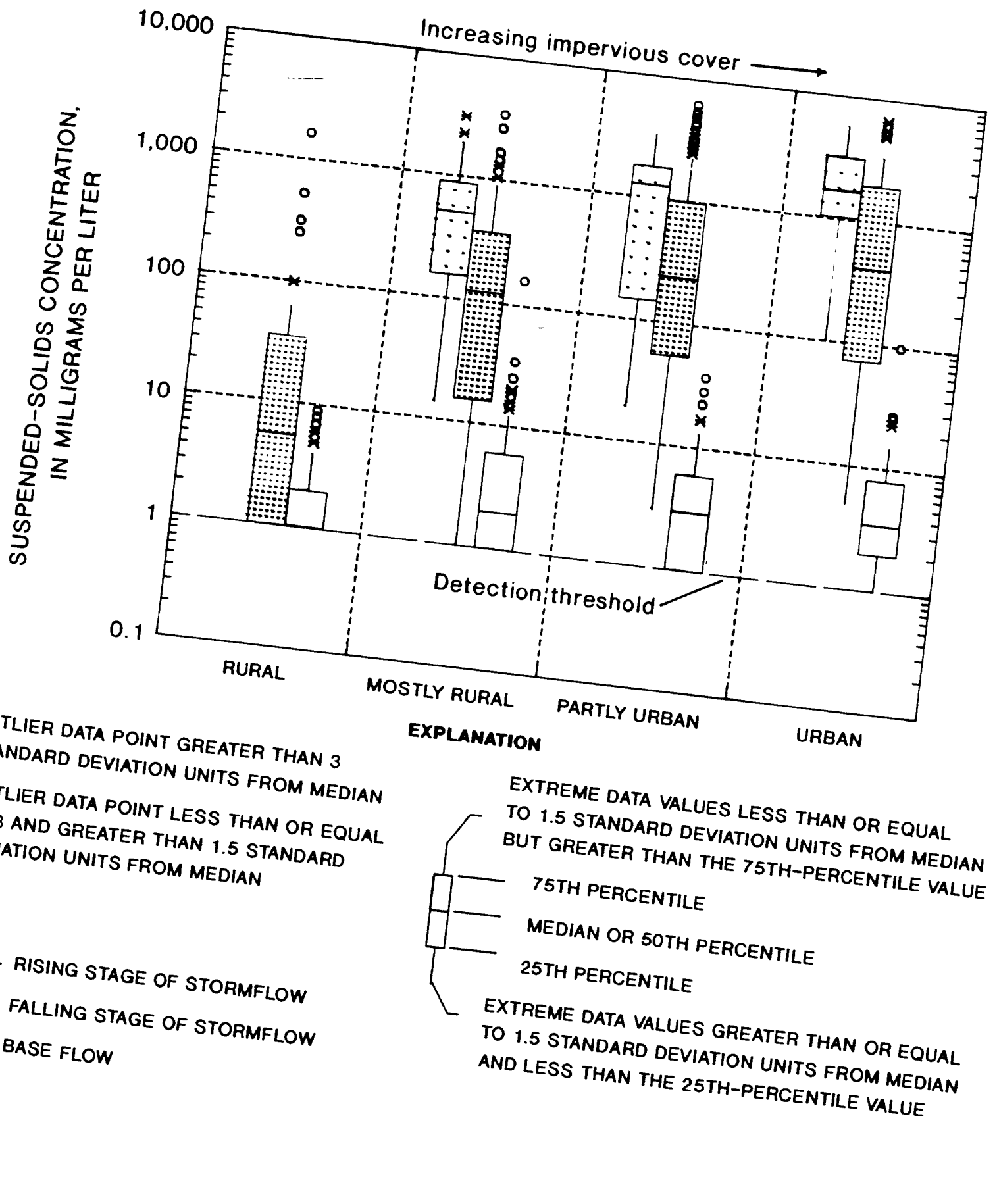

Figure 23

classification, of suspended-solids concentrations. 
Table 8.--Median concentrations and densities of selected water-quality properties and constituents for four development classifications

$[\mathrm{mg} / \mathrm{L}$, milligrams per liter; cols. $/ 100 \mathrm{~mL}$, colonies per 100 milliliters;

--, not detected; ( ), number in parentheses represents number of samples!

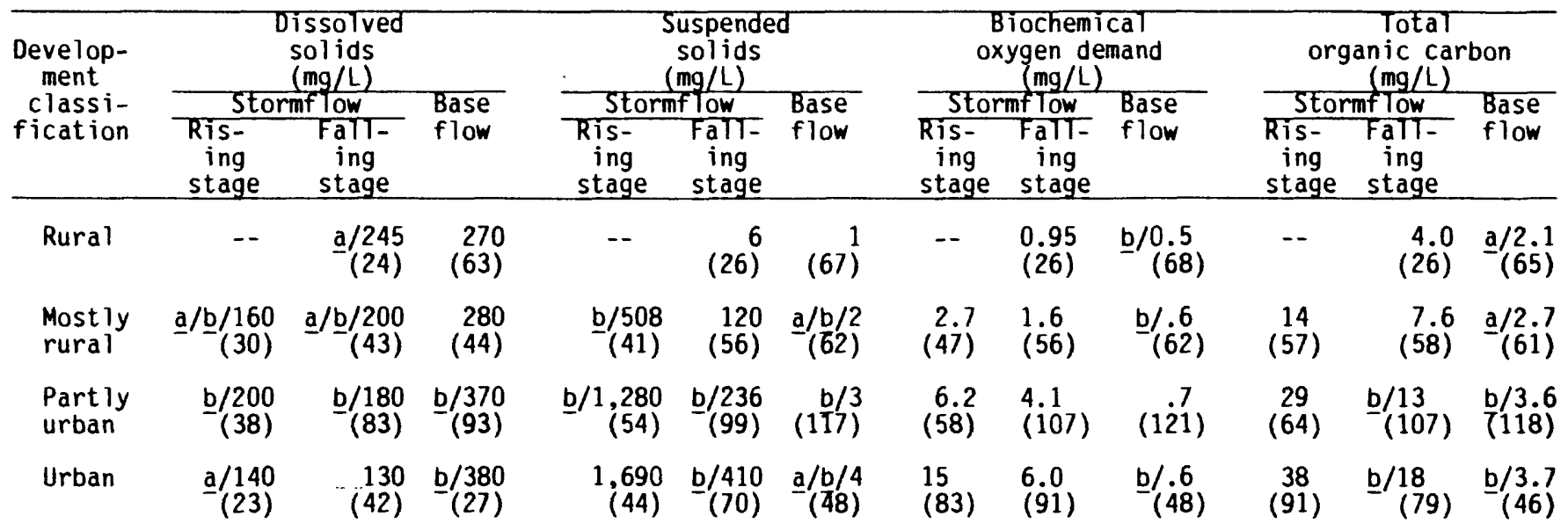

\begin{tabular}{|c|c|c|c|c|c|c|c|c|c|c|c|c|}
\hline \multirow{2}{*}{$\begin{array}{l}\text { Develop- } \\
\text { ment } \\
\text { classi- } \\
\text { fication }\end{array}$} & \multicolumn{3}{|c|}{$\begin{array}{c}\text { Total } \\
\text { nitrogen } \\
(\mathrm{mg} / \mathrm{L})\end{array}$} & \multicolumn{3}{|c|}{$\begin{array}{c}\text { Total } \\
\text { phosphorus } \\
(\mathrm{mg} / \mathrm{L})\end{array}$} & \multicolumn{3}{|c|}{$\begin{array}{c}\text { Fecal } \\
\text { coliforms } \\
\text { (cols. }(100 \mathrm{~mL}) \\
\end{array}$} & \multicolumn{3}{|c|}{$\begin{array}{c}\text { Fecal } \\
\text { streptococci } \\
\text { (cols. } / 100 \mathrm{~mL}) \\
\end{array}$} \\
\hline & \begin{tabular}{l}
\multicolumn{1}{c}{ Stor } \\
Ris- \\
ing \\
stage
\end{tabular} & $\begin{array}{l}\text { flow } \\
\text { Fall- } \\
\text { ing } \\
\text { stage }\end{array}$ & $\begin{array}{l}\text { Base } \\
\text { flow }\end{array}$ & $\begin{array}{c}\text { Stor } \\
\text { Ris- } \\
\text { ing } \\
\text { stage }\end{array}$ & $\begin{array}{l}\text { nf Tow } \\
\text { FaTT- } \\
\text { ing } \\
\text { stage }\end{array}$ & $\begin{array}{l}\text { Base } \\
\text { flow }\end{array}$ & $\begin{array}{l}\text { Storm } \\
\text { Ris- } \\
\text { ing } \\
\text { stage }\end{array}$ & $\begin{array}{c}\text { Tow } \\
\text { Fant- } \\
\text { ing } \\
\text { stage }\end{array}$ & $\begin{array}{l}\text { Base } \\
\text { flow }\end{array}$ & $\begin{array}{c}\text { Storn } \\
\text { Ris- } \\
\text { ing } \\
\text { stage }\end{array}$ & $\begin{array}{c}\text { Fow } \\
\text { FaIT- } \\
\text { ing } \\
\text { stage }\end{array}$ & $\begin{array}{l}\text { Base } \\
\text { flow }\end{array}$ \\
\hline Rural & -- & $\begin{array}{l}0.50 \\
(26)\end{array}$ & $\begin{array}{l}0.31 \\
(67)\end{array}$ & -- & $\begin{array}{r}0.020 \\
(26)\end{array}$ & $\underline{b} / 0.010$ & -- & $\begin{array}{r}1,000 \\
(20)\end{array}$ & $\begin{array}{l}b / 40 \\
7(30)\end{array}$ & -- & $\begin{array}{r}1,200 \\
(20)\end{array}$ & $\begin{array}{l}\mathrm{b} / 90 \\
(30)\end{array}$ \\
\hline $\begin{array}{l}\text { Mostly } \\
\text { rural }\end{array}$ & $\begin{array}{l}1.60 \\
(55)\end{array}$ & $\begin{array}{l}1.15 \\
(58)\end{array}$ & $\begin{array}{l}.44 \\
(59)\end{array}$ & $\begin{array}{r}0.120 \\
(55)\end{array}$ & $\begin{array}{l}.055 \\
(58)\end{array}$ & $\begin{array}{r}\mathrm{b} / .010 \\
(63)\end{array}$ & $\underline{b} / 22,000$ & $\begin{array}{r}3,700 \\
(53)\end{array}$ & $\begin{array}{l}b / 41 \\
(63)\end{array}$ & $\begin{array}{r}29,000 \\
(47)\end{array}$ & $\begin{array}{r}7,600 \\
(53)\end{array}$ & $\begin{array}{l}b / 88 \\
(63)\end{array}$ \\
\hline $\begin{array}{l}\text { Partly } \\
\text { urban }\end{array}$ & $\begin{array}{r}b / 3.60 \\
(69)\end{array}$ & $\begin{array}{r}\mathrm{b} / 2.00 \\
(110)\end{array}$ & $\begin{array}{l}\text { b/.70 } \\
\text { (119) }\end{array}$ & $\begin{array}{l}.560 \\
(69)\end{array}$ & (110) & $c / .030$ & $\begin{array}{r}b / 24,500 \\
(58)\end{array}$ & $\begin{array}{r}30,000 \\
(95)\end{array}$ & $\begin{array}{r}180 \\
(95)\end{array}$ & $\begin{array}{r}54,000 \\
(58)\end{array}$ & $\begin{array}{r}\mathrm{a} / 48,000 \\
(96)\end{array}$ & $\begin{array}{r}260 \\
(95)\end{array}$ \\
\hline Urban & $\begin{array}{r}\mathrm{b} / 4.30 \\
(91)\end{array}$ & $\begin{array}{r}b / 2.15 \\
(86)\end{array}$ & b/.87 & $\begin{array}{r}1.350 \\
(92)\end{array}$ & $\begin{array}{l}450 \\
(88)\end{array}$ & $\underline{c} / .040$ & $\begin{array}{r}110,000 \\
(67)\end{array}$ & $\begin{array}{r}42,000 \\
(69)\end{array}$ & $\begin{array}{r}1,200 \\
(48)\end{array}$ & $\begin{array}{r}180,000 \\
(66)\end{array}$ & $\begin{array}{r}\mathrm{a} / 75,000 \\
(70)\end{array}$ & $\begin{array}{r}650 \\
(48)\end{array}$ \\
\hline
\end{tabular}

a/ For each flow category, median not significantly different from medians of other development classifications at $\alpha$ of $>0.05$.

b/ Median not significantly different from medians of other development classifications at $\alpha$ of $>0.10$. c/ Median not significantly different from medians of other development classifications at $\alpha$ of $>0.10$. 
median for the rural classification was significantly different from those of the three other classifications, and the base-flow median for mostly rural was significantly different from that of urban at $\alpha<0.10$. Although more difficult to determine because of the logarithmic scale, the interquartile range (a robust estimator of the variability) increased with increasing development classification, except for the rising stage of stormflow. The variability represented by the interquartile ranges was larger in the rising stage than in the falling stage; the variability of the ranges in both stages of stormflow exceeded that in base flow significantly.

The median concentrations and variability of $B O D$ increased with increasing development (fig. 24, table 8). The BOD medians in the rising stage and falling stage of stormflow for the development classifications were significantly different at a $<0.05$. Distributions of concentrations of BOD in base flow were not significantly different except for the difference between partly urban and the other development classifications. Variability represented by the interquartile ranges decreased from the rising stage to the falling stage of stormflow, and from the falling stage to base flow.

The median concentrations of TOC in the rising stage and falling stage of stormflow increased with increasing development; however, no statistical difference exists between urban and partly urban classifications in the falling stage (fig. 25, table 8). The variability generally increased with increasing development. Within flow categories, variability decreased from the rising stage to the falling stage, and from the falling stage to base flow. Base-flow medians were significantly larger for urban and partly urban than for rural and mostly rural, but the differences were much smaller than the corresponding differences between medians in the two stages of stormflow.

Median concentrations of total nitrogen increased with increasing development (fig. 26, table 8). The median concentrations from all development classification were significantly different from each other in the rising stage and falling stage of stormflow and in base flow except for the median concentrations from urban and partly urban basins in all flow categories. The variability increased with increasing development except in the rising stage and in base flow for the urban basins. Within each development classification, the variability was larger in the rising stage than in the falling stage, and larger in both stages of stormflow than in base flow.

Median concentrations of total phosphorus in the rising stage and falling stage of stormflow significantly increased with increasing development (fig. 27 , table 8 ). The variability increased with development classification. The variability in the rising stage exceeded that in the falling stage of stormflow and the variability in both stages of stormflow exceeded that in base flow. Base-flow median concentrations for rural and mostly rural development classifications were significantly less than the medians for urban and partly urban classifications, but the differences were much smaller than the corresponding differences between median concentrations in the two stages of stormflow.

The medians of fecal-coliform densities increased with increasing development in both stages of stormflow and in base flow (fig. 28). Variability increased with development classification. Except for the median densities from the partly urban classification, the variability decreased from the rising stage to the falling stage of stormflow and from both stages of 


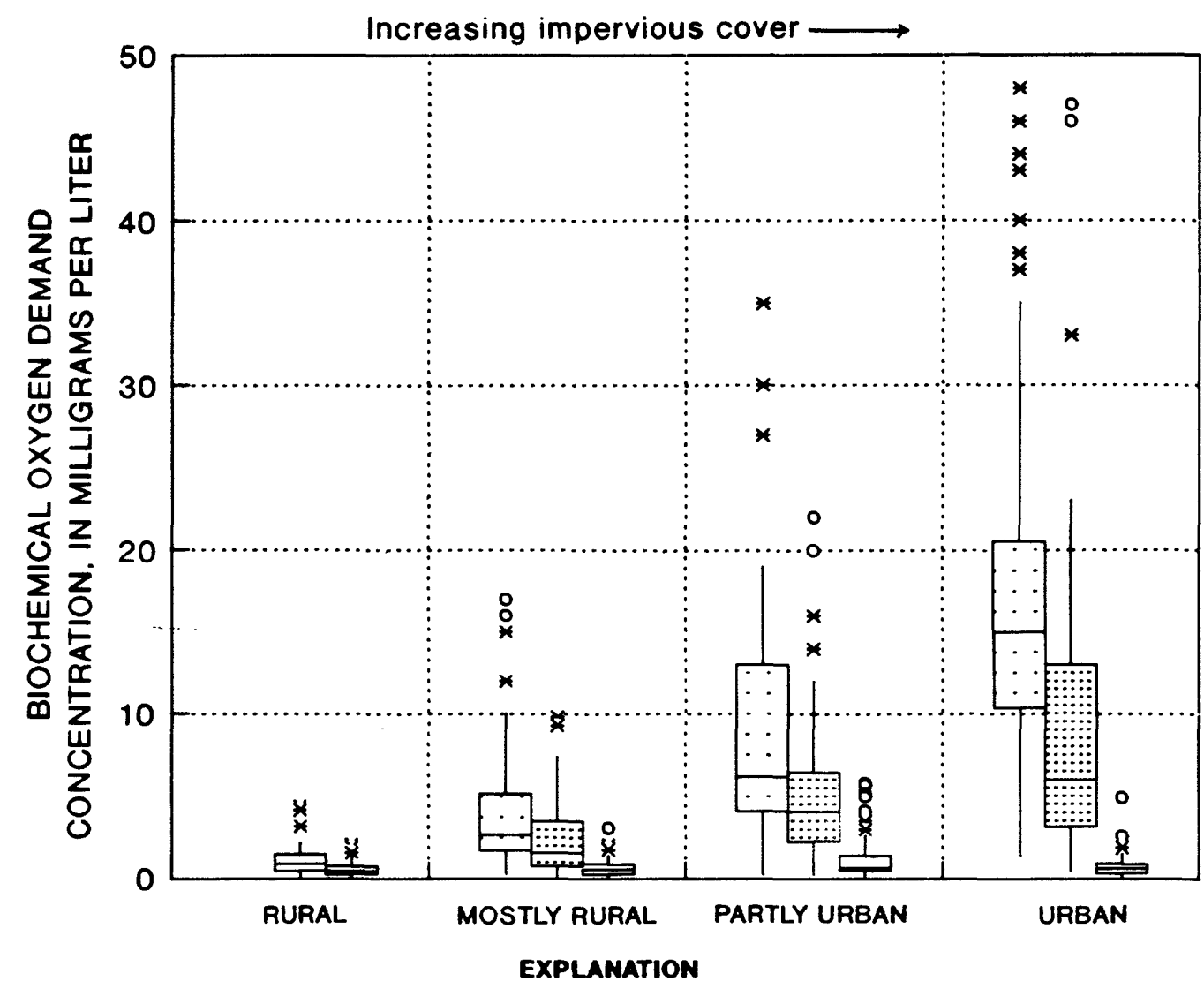

- OUTLIER DATA POINT GREATER THAN 3

EXTREME DATA VALUES LESS THAN OR EQUAL STANDARD DEVIATION UNITS FROM MEDIAN TO 1.5 STANDARD DEVIATION UNITS FROM MEDIAN

* outlier data point less than or equal TO 3 AND GREATER THAN 1.5 STANDARD DEVIATION UNITS FROM MEDIAN BUT GREATER THAN THE 75TH-PERCENTILE VALUE

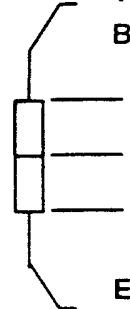

75TH PERCENTILE

MEDIAN OR 50TH PERCENTILE

25TH PERCENTILE

EXTREME DATA VALUES GREATER THAN OR EQUAL TO 1.5 STANDARD DEVIATION UNITS FROM MEDIAN AND LESS THAN THE 25TH-PERCENTILE VALUE

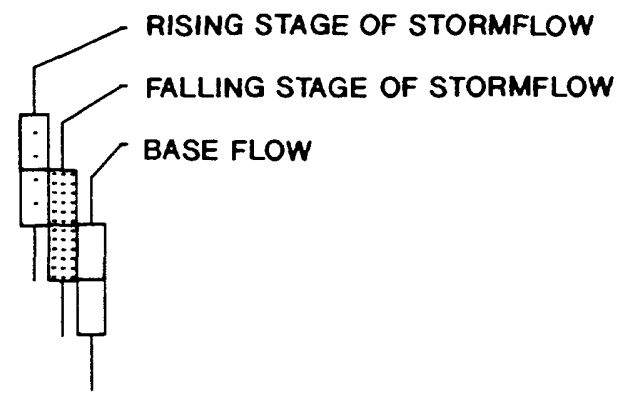

Figure 24.--Boxplots showing the distribution, by flow category and development classification, of biochemical oxygen demand concentrations. 


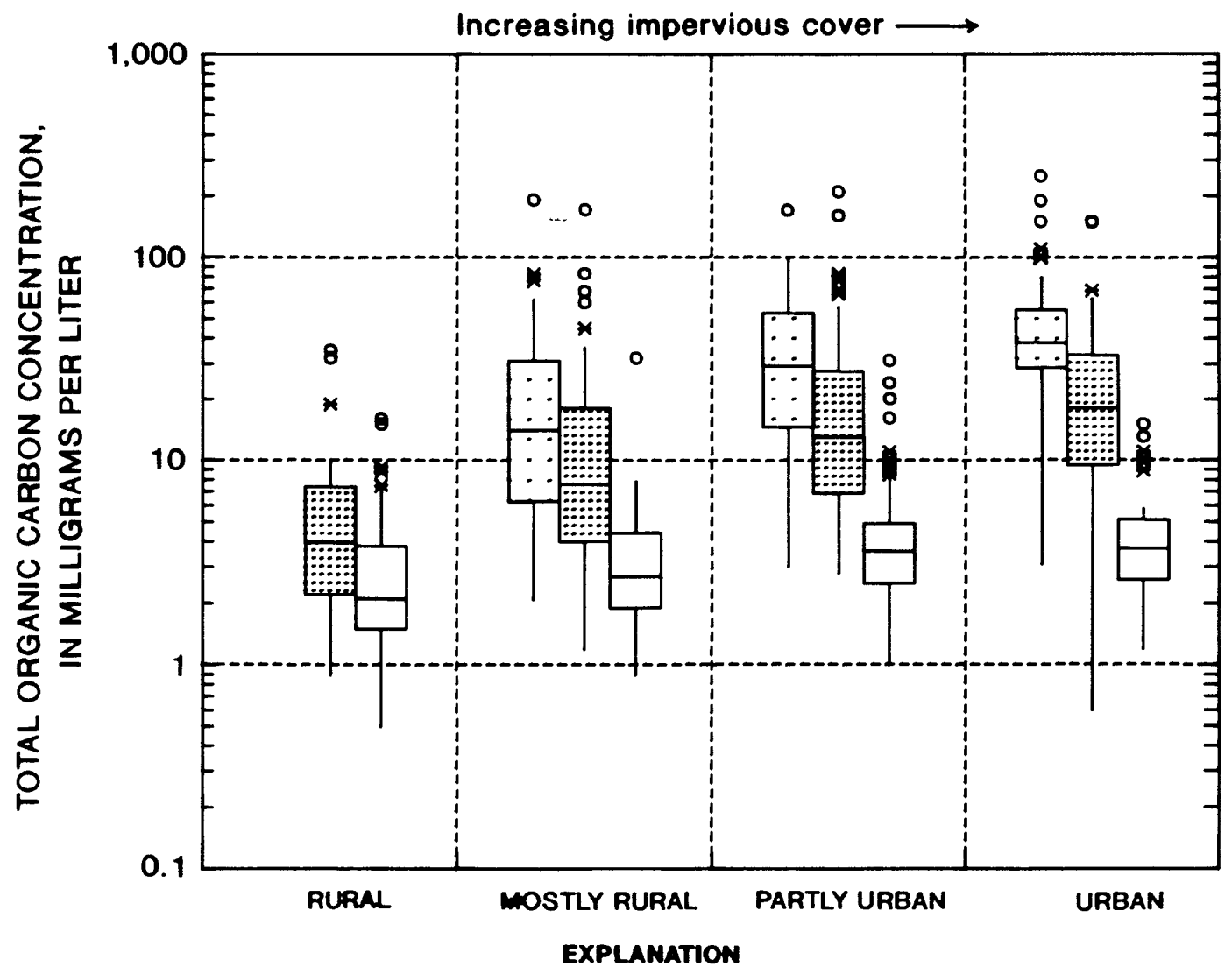

- OUTLIER DATA POINT GREATER THAN 3 STANDARD DEVIATION UNITS FROM MEDIAN

EXTREME DATA VALUES LESS THAN OR EQUAL TO 1.5 STANDARD DEVIATION UNITS FROM MEDIAN OUTLIER DATA POINT LESS THAN OR EQUAL TO 3 AND GREATER THAN 1.5 STANDARD DEVIATION UNITS FROM MEDIAN
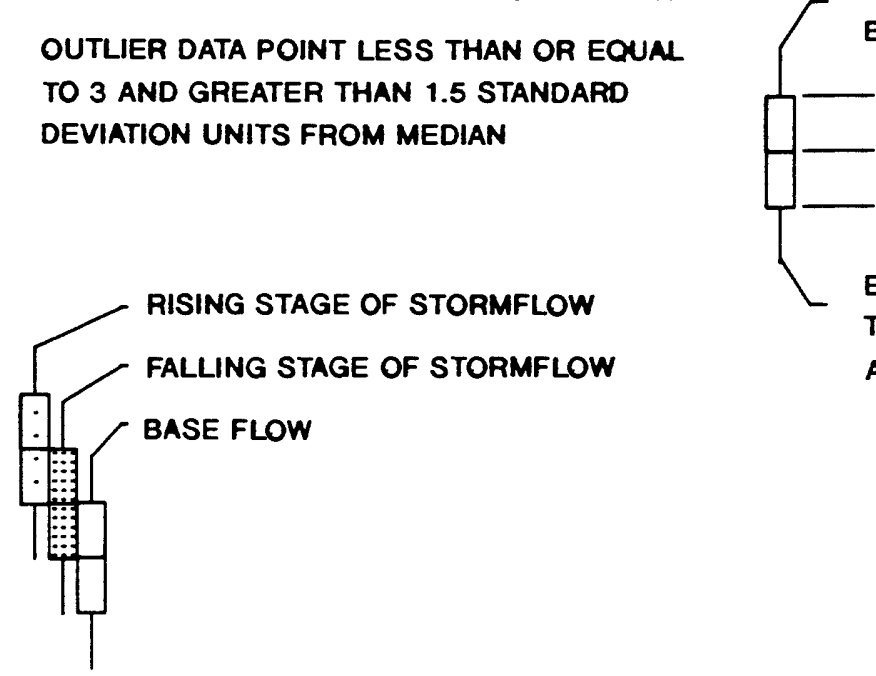
BUT GREATER THAN THE 75TH-PERCENTILE VALUE 75TH PERCENTILE MEDIAN OR 50TH PERCENTILE 25TH PERCENTILE

EXTREME DATA VALUES GREATER THAN OR EQUAL TO 1.5 STANDARD DEVIATION UNITS FROM MEDIAN AND LESS THAN THE 25TH-PERCENTILE VALUE

Figure 25.--Boxplots showing the distribution, by flow category and development classification, of total organic carbon concentrations. 


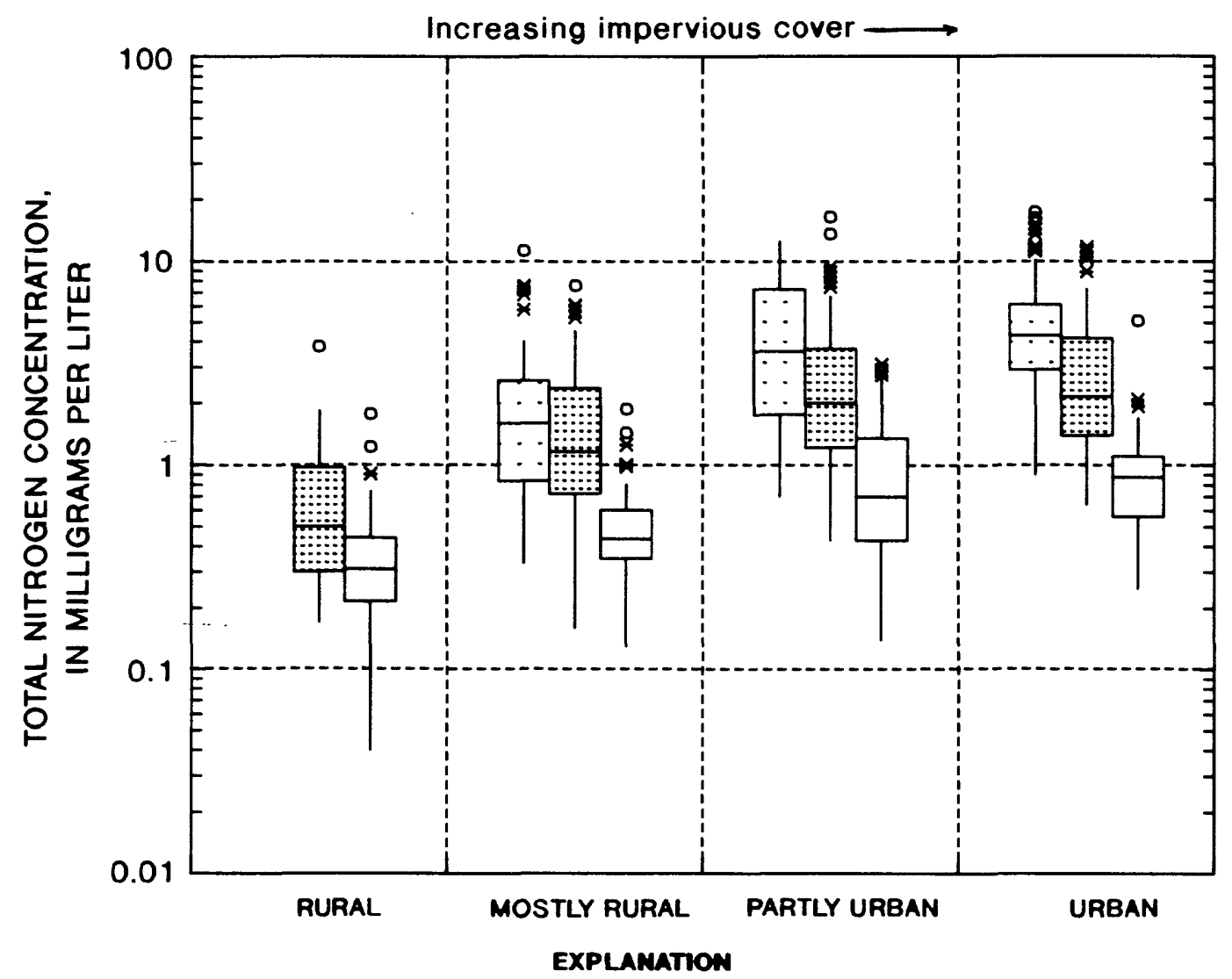

- OUTLIER DATA POINT GREATER THAN 3

EXTREME DATA VALUES LESS THAN OR EQUAL STANDARD DEVIATION UNITS FROM MEDIAN TO 1.5 STANDARD DEVIATION UNITS FROM MEDIAN BUT GREATER THAN THE 75TH-PERCENTILE VALUE

* outlier data pont less than or equal TO 3 AND GREATER THAN 1.5 STANDARD DEVIATION UNITS FAOM MEDIAN
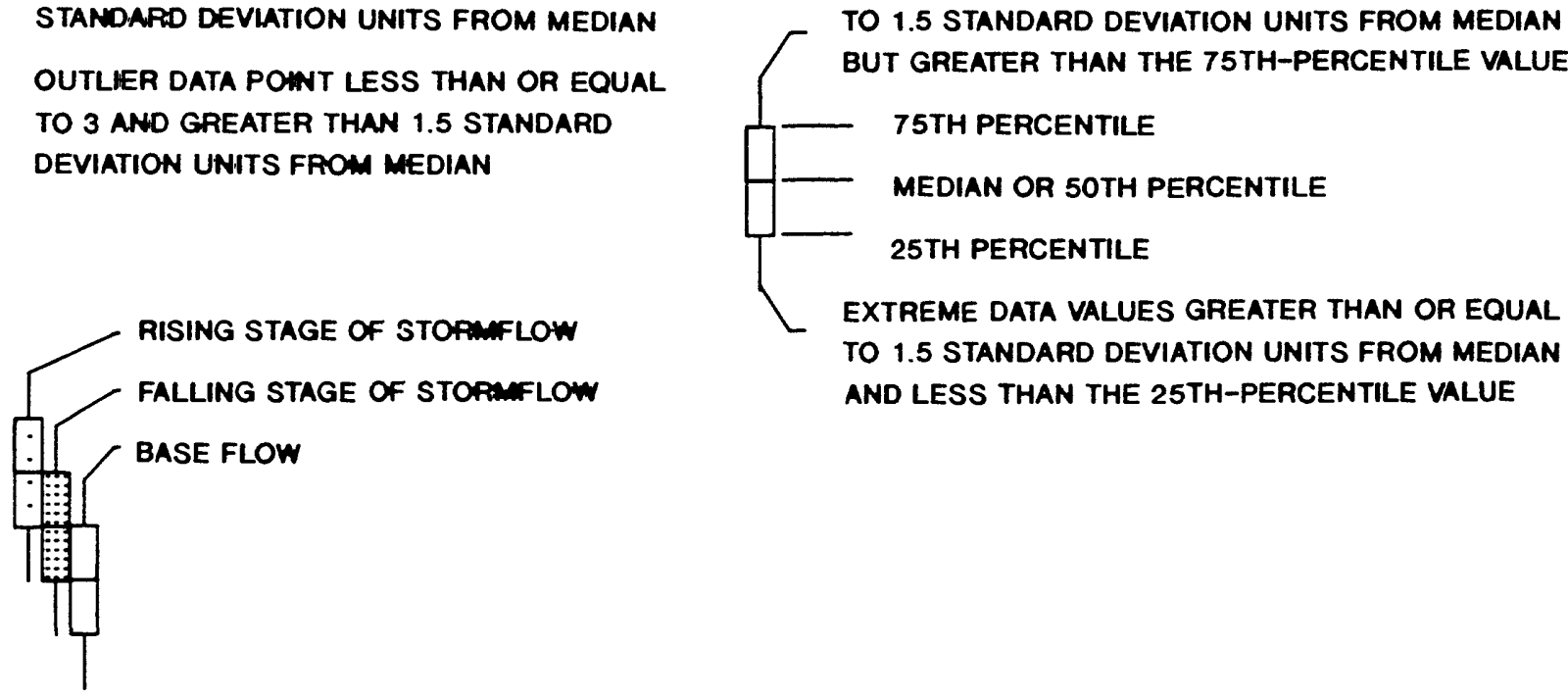
AND LESS THAN THE 25TH-PERCENTILE VALUE

Figure 26.--Boxplots showing the distribution, by flow category and development classification, of total nitrogen concentrations. 


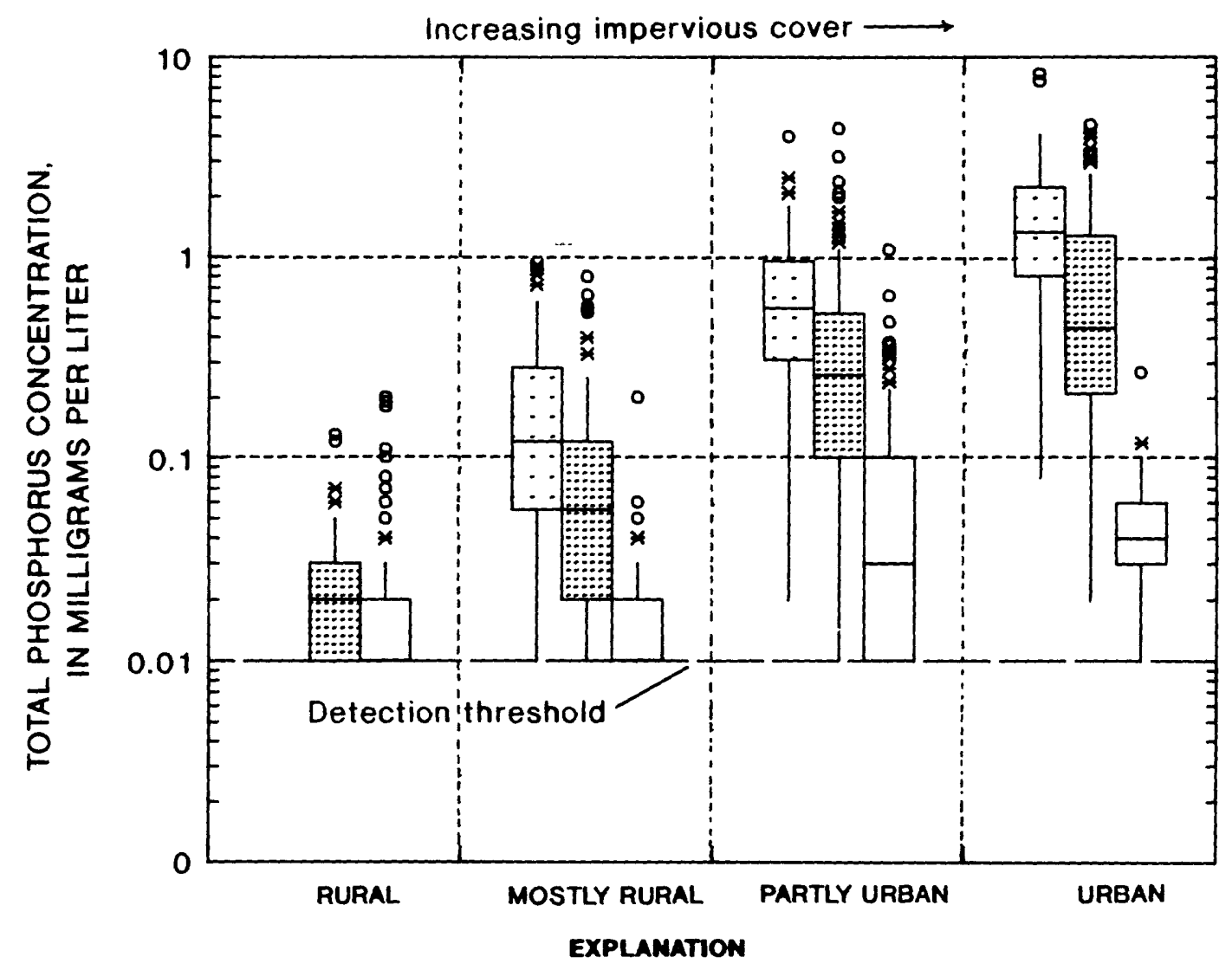

- OUTLIER DATA POINT GREATER THAN 3 STANDARD DEVIATION UNITS FROM MEDIAN

* outlier data point less than or equal TO 3 AND GREATER THAN 1.5 STANDARD DEVIATION UNITS FROM MEDIAN
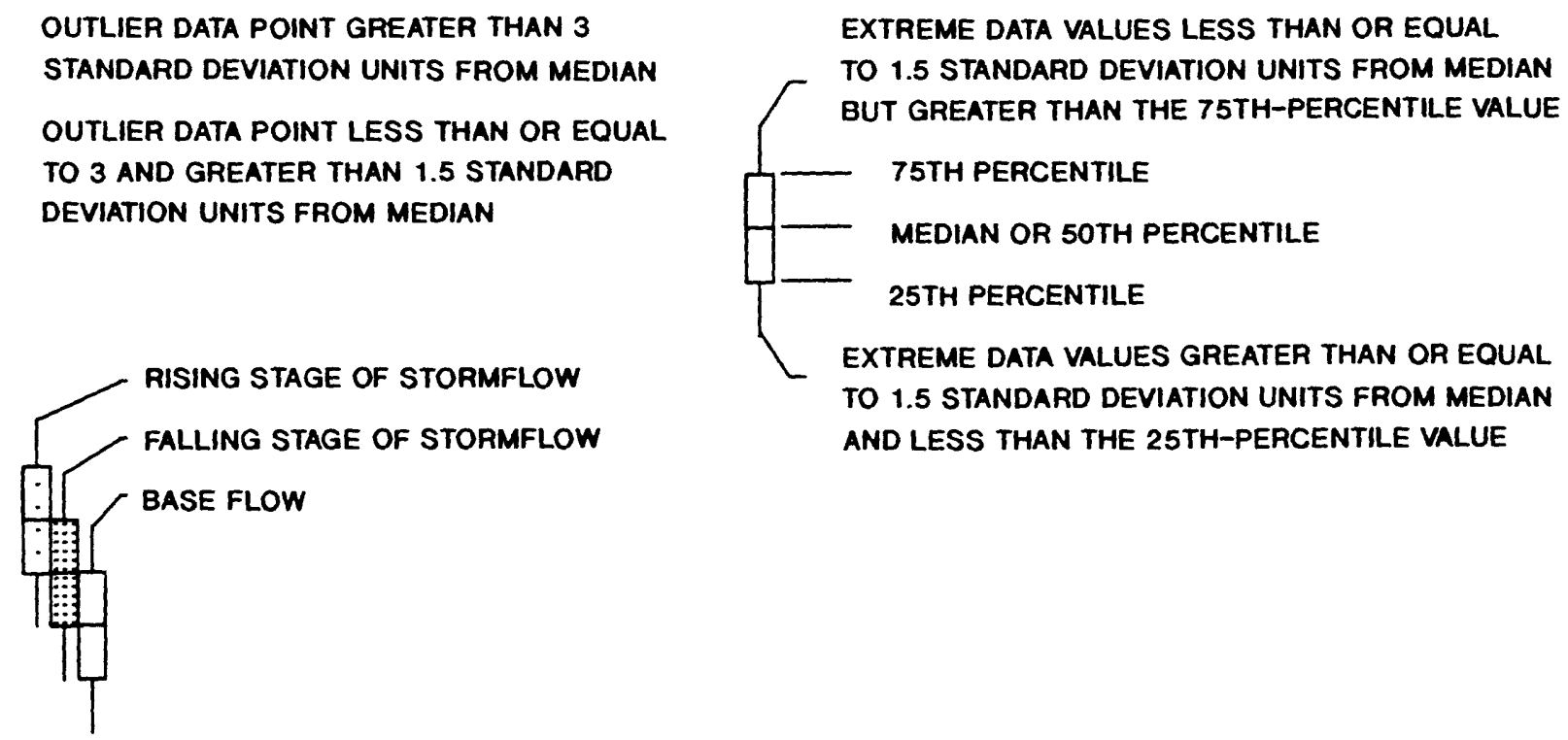

Figure 27.--Boxplots showing the distribution, by flow category and development classification, of total phosphorus concentrations. 


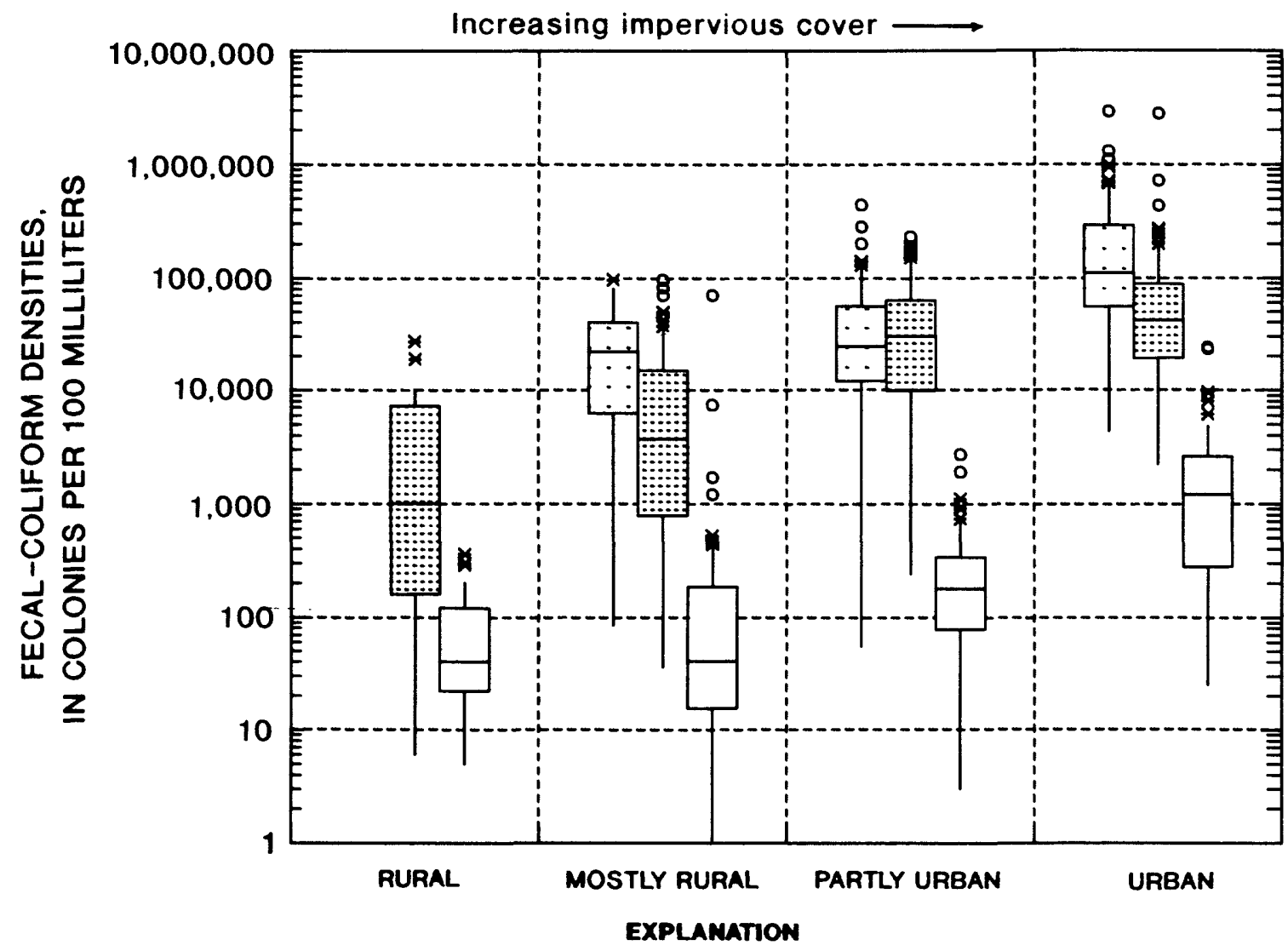

- OUTLIER DATA POINT GREATER THAN 3 STANDARD DEVIATION UNITS FROM MEDIAN

EXTREME DATA VALUES LESS THAN OR EQUAL TO 1.5 STANDARD DEVIATION UNITS FROM MEDIAN OUTLIER DATA POINT LESS THAN OR EQUAL TO 3 AND GREATER THAN 1.5 STANDARD DEVIATION UNITS FROM MEDIAN BUT GREATER THAN THE 75TH-PERCENTILE VALUE

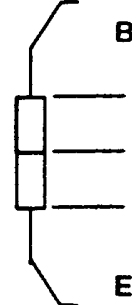

75TH PERCENTILE

MEDIAN OR 5OTH PERCENTILE 25TH PERCENTILE

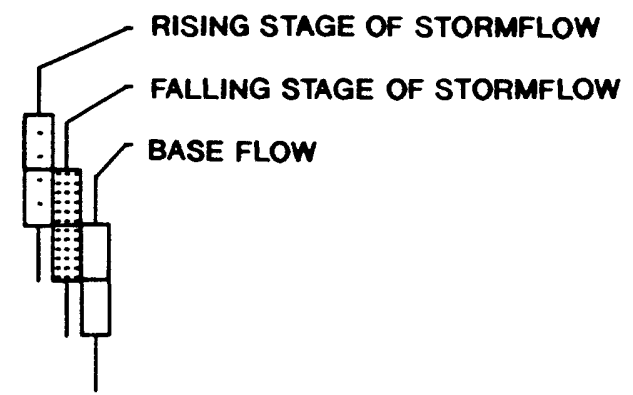

EXTREME DATA VALUES GREATER THAN OR EQUAL TO 1.5 STANDARD DEVIATION UNITS FROM MEDIAN AND LESS THAN THE 25TH-PERCENTILE VALUE

Figure 28.--Boxplots showing the distribution, by flow category and development classification, of fecal-coliform densities. 
stormflow to base flow. Base-flow median densities for the rural and mostly rural classifications are not significantly different from each other, but the differences between medians for the other classifications are significant.

The median densities of fecal streptococci in both stages of stormflow generally increased with increasing development at $\alpha<0.05$; the urban and partly urban classifications differed significantly at $\alpha<0.10$ in the falling stage of stormflow (fig. 29, table 8). Variability increased with increasing development, and from base flow to the falling stage to the rising stage of stormflow. All the differences between base-flow median densities are significant except the difference between medians from the rural and mostly rural classifications. Although significant statistically, the differences in base-flow median densities between the classifications are not as large in base flow as are these differences between the classifications in the two stages of stormflow.

Analyses of the 12 dissolved inorganic trace elements in samples from the 18 sites also were combined into four groups based on the development classification of the upstream drainage basin for the purpose of statistical comparison. Only two of the elements, dissolved arsenic and dissolved barium, were detected in a sufficient number of samples for statistical comparison of the concentrations among the development classifications. Concentrations of dissolved arsenic and dissolved barium were significantly larger for the partly urban and urban development classifications.

Dissolved copper, dissolved iron, dissolved manganese, and dissolved zinc had a large portion of analyses with concentrations less than the multiple detection thresholds, so statistical differences between concentrations could not be established. For these four constituents, the proportion of analyses with concentrations greater than the largest detection threshold divided by the total number of analyses for each of the four development classifications were compared using the chi square distribution. Dissolved copper had too few values greater than the largest detection threshold for comparison between the groups. The number of times the concentration of dissolved iron was more than the largest detection threshold was significantly greater for urban and partiy urban classificaitons. For manganese, the partly urban classification had significantly more concentrations than the three other classifications mainly because of the large concentrations collected at site 16. Dissolved zinc did not have a statistically greater portion of samples that were larger than the largest detection threshold for any development classification.

Concentrations of 42 synthetic organics in samples collected from the 18 sites were also combined into four groups based on the development classifications for statistical comparison. Eleven insecticides and herbicides were not detected in a sufficient number of samples for statistical comparison of concentrations. For these constituents, the ratios of those samples with detectable concentrations divided by the total number of samples analyzed for each of the four development classifications were compared using chi square distribution. of the organochlorine compounds, chlordane, DDD, DDE, DDT, and dieldrin all had ratios from the urban classification that were significantiy larger than the ratios from the other three classifications. For diazinon and malathion, the urban classification had a significantly larger ratio than did the partiy urban classification, which, in turn, had a significantly larger ratio than did the rural and mostly rural classifications. For the chlorophenoxy acid compounds, 2,4-D and 2,4,5-T had significantly larger ratios in the urban classifications than in the rural classifications. For 


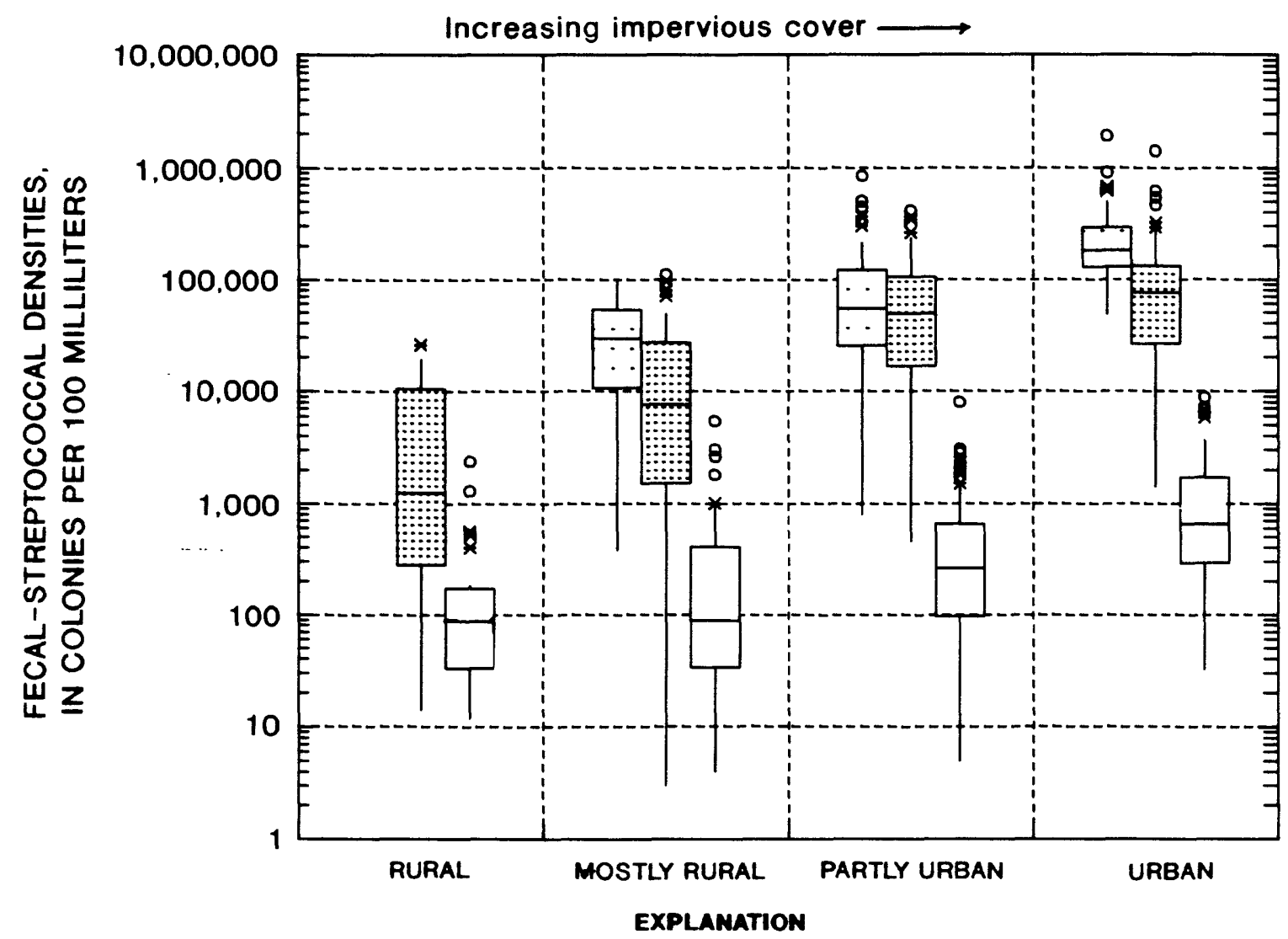

c OUTLIER DATA POINT GREATER THAN 3 STANDARD DEVIATION UNITS FROM MEDIAN

EXTREME DATA VALUES LESS THAN OR EQUAL TO 1.5 STANDARD DEVIATION UNITS FROM MEDIAN

* outlier data point less than or equal TO 3 AND GREATER THAN 1.5 STANDARD DEVIATION UNITS FROM MEDIAN BUT GREATER THAN THE 75TH-PERCENTILE VALUE

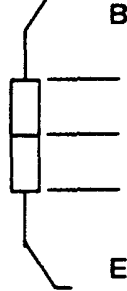

75TH PERCENTILE MEDIAN OR 5OTH PERCENTILE 25TH PERCENTILE

RISING STAGE OF STORMFLOW FALLING STAGE OF STORMFLOW EXTREME DATA VALUES GREATER THAN OR EQUAL TO 1.5 STANDARD DEVIATION UNITS FROM MEDIAN AND LESS THAN THE 25TH-PERCENTILE VALUE

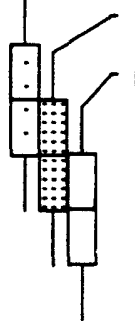
BASE FLOW

Figure 29.--Boxplots showing the distribution, by flow category and development classification, of fecal-streptococcal densities. 
atrazine, the partly urban and urban classifications had significantly larger ratios than did the rural and mostly rural classifications. Prometone was detected in a significantly larger proportion of samples from the urban classification than from the partly urban classification and in a significantly larger proportion of samples from the partly urban classification than from the mostly rural and rural classifications.

\section{SUMMARY}

Selected water-quality constituents of stormflow and base flow at 18 sites in the Austin area were analyzed to determine the relation between the extent of drainage-bas in development and water quality. Boxplots illustrating the distribution of the concentrations of eight constituents in rising stage and falling stage of stormflow and in base flow were constructed for each of the sample sites. Except for dissolved solids, the concentrations in samples collected during the rising stage were larger than those in the falling stage. Concentrations in samples from the rising stage and falling stage of stormflow were larger than those from base flow. The concentrations of suspended solids, BOD, TOC, total nitrogen, total phosphorus, fecal coliforms, and fecal streptococci were larger in stormflow than in base flow. The sites with runoff from urban drainage basins had larger concentrations than the sites draining rural basins in each of the three flow categories.

The 18 inorganic trace elements for which samples were analyzed were detected at 1 or more of the 18 sample sites. Most samples with detectable amounts and larger concentrations of many of these trace elements were collected at the sites with the more urban drainage basins. Twenty-two of the 42 selected synthetic organic compounds analyzed were detected more often and in larger concentrations at sample sites with more urban basins.

Statistical comparisons were made to substantiate differences in water quality among the five sites with automatic samplers. The concentrations in samples collected during the rising stage of stormflow were more variable than those in samples collected during the falling stage. Concentrations in samples collected during stormflow were more variable than those in samples collected during base flow. The median concentrations in both the rising stage and falling stage of stormflow increased from the site with a mostly rural drainage basin to the two sites with urban basins. The differences between median concentrations in samples from the rising stage and the falling stage were proportionally larger for the more urban drainage basins. The differences between the median concentrations in samples from the rising stage and falling stage of stormflow were statistically significant for the mostly rural and partly urban drainage basins.

Statistical comparisons were made to substantiate differences between concentrations of constituents in samples collected during each flow category at sites grouped by development classification. Except for dissolved solids, the median concentrations in samples from the more developed sites were significantly larger in both stages of stormflow than in base flow. Although the actual difference between the concentrations in base flow were smaller among the classifications, the group medians were significantly larger, in most cases, for the groups of more developed sites. Similarly, the variability of the concentrations generally increased from the groups of less developed sites to the more developed sites and from base flow to falling stage of stormflow to rising stage of stormflow. 


\section{SELECTED REFERENCES}

Andrews, F.L., Schertz, T.L., Slade, R.M., Jr., and Rawson, Jack, 1984, Effects of storm-water runoff on water quality of the Edwards aquifer near Austin, Texas: U.S. Geological Survey Water-Resources Investigations Report 84-4124, $50 \mathrm{p}$.

Baker, E.T., Jr., Slade, R.M., Jr., Dorsey, M.E., Ruiz, L.M., and Duffin, G.L., 1986, Geohydrology of the Edwards aquifer in the Austin area, Texas: Texas Water Development Board Report 293, 217 p.

Blevins, D.W., 1984 [1986], Quality of stormwater runoff in the Blue River basin, Missouri and Kansas, July-October 1981 and April-July 1982: U.S. Geological Survey Water-Resources Investigations Report 84-4226, $131 \mathrm{p}$.

Carr, J.T., Jr., 1967, The climate and physiography of Texas: Texas Water Development Board Report 53, 17 p.

Choffel, K.L., Roddy, W.R., and Mitchell, R.N., 1977, Hydrologic data for urban studies in the Austin, Texas, metropolitan area, 1975: U.S. Geological Survey Open-File Report 76-885, 126 p.

City of Austin, Department of Environmental Protection, 1986, City of Aust in stormwater monitoring program description: $21 \mathrm{p}$.

City of Austin, Planning Department, 1982, Demographic and land-use projections and distribution for the growth management scenario: $23 \mathrm{p}$.

City of Austin, Watershed Management Division, Public Works Department, 1984, Stormwater quality modeling study for Austin creeks: $40 \mathrm{p}$.

Ellis, S.R., and Alley, W.M., 1979, Quantity and quality of urban runoff from three localities in the Denver metropolitan area, Colorado: U.S. Geological Survey Water-Resources Investigations Report 79-64, $67 \mathrm{p}$.

Ellis, S.R., Linder, J.B., and Patterson, D.A., 1983, Effects of increased urbanization on the storm runoff from a small urban basin in metropolitan Denver, Colorado: International Symposium on Urban Hydrology, Hydraulics, and Sediment Control, University of Kentucky, Lexington, Kentucky, p. 79-86.

Engineering-Science, Inc., 1982, Draft final report of the Nationwide Urban Runoff Program in Austin, Texas: Austin, Texas, $185 \mathrm{p}$.

Farnsworth, R.K., 1982, Mean monthly, seasonal, and annual pan evaporation for the United States: U.S. Department of Commerce, National Oceanic and Atmospheric Administration, National Weather Service, Technical Report 34, 82 p.

Fisher, G.T., and Katz, B.G., 1984, Analyses of urban storm-water runoff characteristics of four basins in the Baltimore metropolitan area, Maryland: U.S. Geological Survey Water-Resources Investigations Report $84-4099,58 \mathrm{p}$.

Forstner, U., and Wittmann, G.T.W., 1983, Metal pollution in the aquatic environment, 2nd edition: New York, Springer-Verlag, 486 p.

Garner, L.E., and Young, K.P., 1976, Environmental geology of the Aust in area: an aid to urban planning: Austin, University of Texas, Bureau of Economic Geology Report of Investigations 86, $39 \mathrm{p}$.

Geldreich, E.E., and Kenner, B.A., 1969, Concepts of fecal streptococci in stream pollution: Journal of the Water Pollution Control Federation, v. 41, pt. 2, p. A336-352.

Gordon, J.D., Pate, D.L., and Dorsey, M.E., 1985, Hydrologic data for urban studies in the Austin, Texas, metropolitan area, 1983: U.S. Geological Survey Open-File Report 85-172, $154 \mathrm{p}$.

-.-- 1986, Hydrologic data for urban studies in the Austin, metropolitan area, Texas, 1984: U.S. Geological Survey Open-File Report 85-676, 92 p.

-..- 1987, Hydrologic data for urban studies in the Austin metropolitan area, Texas, 1985: U.S. Geological Survey Open-File Report 87-224, 170 p. 
Gordon, J.D., Pate, D.L., and Slagle, D.L., 1988, Hydrologic data for urban studies in the Aust in metropolitan area, Texas, 1986: U.S. Geological Survey Open File Report 87-768, 144 p.

Harms, L.L., Smith, M., and Goddard, K., 1983, Urban runoff control in Rapid City, South Dakota: South Dakota School of Mines and Technology report, $153 \mathrm{p}$.

Helsel, D.R., and Cohn, T.A., 1988, Estimation of descriptive statistics for multiply-censored water-quality data: Water Resources Research, $v$. 24(12), p. 1997-2004.

Hem, J.D., 1970, Study and interpretation of the chemical characteristics of natural water $(2 d$ ed.): U.S. Geological Survey Water-Supply Paper 1473, $363 \mathrm{p}$.

1985, Study and interpretation of the chemical characteristics of natural water (3rd ed.): U.S. Geological Survey Water-Supply Paper 2254, $264 \mathrm{p}$.

Maderak, M.L., Gordon, J.D., and Mitche11, R.N., 1978, Hydrologic data for urban studies in the Austin, Texas, metropolitan area, 1976: U.S. Geological Survey Open-File Report 78-457, 263 p.

Miller, T.L., and McKenzie, S.W., 1978, Analysis of urban storm-water quality from seven basins near Portland, Oregon: U.S. Geological Survey OpenFile Report 78-662, $81 \mathrm{p}$.

Rawson, Jack, 1974, The quality of surface waters in Texas: U.S. Geological Survey Water-Resources Investigations Open-File Report 7-74, 73 p.

Slade, R.M., Jr., Dorsey, M.E., Gordon, J.D., and Mitchell, R.N., 1980, Hydrologic data for urban studies in the Austin, Texas, metropolitan area, 1978: U.S. Geological Survey Open-File Report 80-728, 229 p.

Slade, R.M., Jr., Dorsey, M.E., Gordon, J.D., Mitche11, R.N., and Gaylord, J.L., 1981, Hydrologic data for urban studies in the Austin, Texas, metropolitan area, 1979: U.S. Geological Survey Open-File Report 81-628, $281 \mathrm{p}$.

Slade, R.M., Jr., Dorsey, M.E., and Stewart, S.L., 1986, Hydrology and water quality of the Edwards aquifer associated with Barton Springs in the Austin area, Texas: U.S. Geological Survey Water-Resources Investigations Report 86-4036, $117 \mathrm{p}$.

Slade, R.M., Jr., Gaylord, J.L., Dorsey, M.E., Mitchel1, R.N., and Gordon, J.D., 1982, Hydrologic data for urban studies in the Austin, Texas, metropolitan area, 1980: U.S. Geological Survey Open-File Report 82-506, $264 \mathrm{p}$.

Slade, R.M., Jr., Gordon, J.D., and Mitche11, R.N., 1979, Hydrologic data for urban studies in the Austin, Texas, metropolitan area, 1977: U.S. Geological Survey Open-File Report 79-271, 192 p.

Slade, R.M., Jr., Veenhuis, J.E., Dorsey, M.E., Gardiner, Heather, and Smith, A.E., 1983, Hydrologic data for urban studies in the Austin, Texas, metropolitan area, 1981: U.S. Geological Survey Open-File Report 83-044, $293 \mathrm{p}$.

Slade, R.M., Jr., Veenhuis, J.E., Dorsey, M.E., Stewart, S.L., and Ruiz, L.M., 1984, Hydrologic data for urban studies in the Austin, Texas, metropolitan area, 1982: U.S. Geological Survey Open-File Report 84-061, $196 \mathrm{p}$.

Smith, J.A., Witkowski, P.J., and Fusillo, T.V., 1987, Manmade organic compounds in the surface waters of the United States; a review of current understanding: U.S. Geological Survey Open-File Report 87-209, 182 p.

Tukey, J.W., 1977, Exploratory data analysis: Reading, Mass., Addison-Wesley, $688 \mathrm{p}$.

U.S. Department of Agriculture, 1974, Soil survey of Travis County, Texas: U.S. Department of Agriculture, $123 \mathrm{p}$. 
U.S. Environmental Protection Agency, 1983, Results of the Nationwide Urban Runoff Program, volume 1, Final report: U.S. Environmental Protection Agency, $186 \mathrm{p}$.

1986a, Maximum contaminant levels (subpart B of part 141, National primary drinking-water regulations): U.S. Code of Federal Regulations, Title 40, parts 100 to 149, revised as of July 1, 1986, p. 521-528.

---- 1986b, Secondary maximum contaminant levels (section 143.3 of part 143, National secondary drinking-water regulations): U.S. Code of Federal Regulations, Title 40, parts 100 to 149 , revised as of July 1, 1986, p. 587-590.

-.--- 1986c, Quality criteria for water, 1986: Office of Water Regulations and Standards, EPA-440/5-86-001, $296 \mathrm{p}$.

U.S. Public Health Service, 1962, Drinking water standards, 1962: U.S. Public Health Service Publication 956, $61 \mathrm{p}$.

Velleman, P.F., and Hoaglin, D.C., 1981, Applications, basics, and computating of exploratory data analysis: Reading, Mass. Addison-Wesley, $354 \mathrm{p}$.

Waddel1, K.M., Massey, B.C. and Jennings, M.E., 1979, Use of the STORM model for estimating the quantity and quality of runoff from the metropolitan area of Houston, Texas: U.S. Geological Survey Water-Resources Investigations Report 79-74, $29 \mathrm{p}$.

Welborn, C.T., and Veenhuis, J.E., 1987, Effects of runoff controls on the quantity and quality of urban runoff at two locations in Austin, Texas: U.S. Geological Survey Water-Resources Investigations Report 87-4004, 101 p. 